Article

\title{
Primary Minerals and Age of The Hydrothermal Quartz Veins Containing U-Mo-(Pb, Bi, Te) Mineralization in the Majerská Valley near Čučma (Gemeric Unit, Spišsko-Gemerské Rudohorie Mts., Slovak Republic)
}

\author{
Štefan Ferenc ${ }^{1, *}$, Martin Števko ${ }^{2,3}{ }^{\mathbb{D}}$, Tomáš Mikuš ${ }^{4}$, Stanislava Milovská $^{4}$, Richard Kopáčik $^{1}$ \\ and Eva Hoppanová ${ }^{1}$ \\ check for \\ updates \\ 1 Department of Geography and Geology, Faculty of Natural Sciences, Matej Bel University, Tajovského 40, \\ 97401 Banská Bystrica, Slovakia; richard.kopacik@umb.sk (R.K.); eva.hoppanova@umb.sk (E.H.) \\ 2 Earth Science Institute of the Slovak Academy of Sciences, Dúbravská cesta 9, 84005 Bratislava, Slovakia; \\ msminerals@gmail.com \\ 3 Department of Mineralogy and Petrology, National Museum, Cirkusová 1740, \\ 19300 Praha 9, Horní Počernice, Czech Republic \\ 4 Earth Science Institute of the Slovak Academy of Sciences, Ďumbierska 1, 97411 Banská Bystrica, Slovakia; \\ mikus@savbb.sk (T.M.); milovska@savbb.sk (S.M.) \\ * Correspondence: stefan.ferenc@umb.sk; Tel.: +421-048-446-7253
}

Citation: Ferenc, Š.; Števko, M.; Mikuš, T.; Milovská, S.; Kopáčik, R.; Hoppanová, E. Primary Minerals and Age of The Hydrothermal Quartz Veins Containing U-Mo-( $\mathrm{Pb}, \mathrm{Bi}, \mathrm{Te})$ Mineralization in the Majerská Valley near Čučma (Gemeric Unit, Spišsko-Gemerské Rudohorie Mts., Slovak Republic). Minerals 2021, 11, 629. https://doi.org/10.3390/ $\min 11060629$

Academic Editors: Jaroslav Pršek and Jiří Sejkora

Received: 7 May 2021

Accepted: 10 June 2021

Published: 13 June 2021

Publisher's Note: MDPI stays neutral with regard to jurisdictional claims in published maps and institutional affiliations.

Copyright: (c) 2021 by the authors. Licensee MDPI, Basel, Switzerland. This article is an open access article distributed under the terms and conditions of the Creative Commons Attribution (CC BY) license (https:// creativecommons.org/licenses/by/ $4.0 /)$.

\begin{abstract}
An occurrence of vein U-Mo mineralization is located in the Majerská valley near Čučma, about $7 \mathrm{~km}$ to the NNE of the district town of Rožňava (Eastern Slovakia). Mineralization is hosted in the acidic metapyroclastics of the Silurian Bystrý Potok Fm. (Gemeric Unit), and originated in the following stages: (I.) quartz I, fluorapatite I; (II.) quartz II, fluorapatite II, zircon, rutile chlorite, tourmaline; (III.) uraninite, molybdenite, U-Ti oxides; (IV.) pyrite I, ullmannite, gersdorffite, cobaltite; (Va.) galena, bismuth, tetradymite, joséite $\mathrm{A}$ and $\mathrm{B}, \mathrm{Bi}_{3}(\mathrm{TeS})_{2}$ mineral phase, $(\mathrm{BiPb})(\mathrm{TeS})$ mineral phase, ikunolite; (Vb.) minerals of the kobellite-tintinaite series, cosalite; (VI.) pyrite II; (VII.) titanite, chlorite; and (VIII.) supergene mineral phases. The chemical in-situ electron-microprobe U-Pb dating of uraninite from a studied vein yielded an average age of around $265 \mathrm{Ma}$, corresponding to the Guadalupian Epoch of Permian; the obtained data corresponds with the age of Gemeric S-type granites. The age correlation of uraninite with the Gemeric S-type granites and the spatial connection of the studied mineralization with the Čučma granite allows us to assume that it is a Hercynian, granite-related (perigranitic) mineralization.
\end{abstract}

Keywords: uranium; vein mineralization; uraninite; molybdenite; sulphotellurides; uraninite dating; Permian; Gemeric Unit; Gemeric granites; Western Carpathians

\section{Introduction}

Radioactive anomalies in the vicinity of the Čučma village, north of the district town Rožňava (Eastern Slovakia), were, in the past, a subject of several repeated geological surveys or mineralogical-geochemical research [1-13]. These works were focused on the prospecting, possible verification of raw materials resources and more detailed genetic or mineralogical-geochemical study of U, REE and Au ores. Unfortunately, the results of the geological survey did not show their economic significance.

The above-mentioned anomalies are related to the hydrothermal quartz (quartzapatite respectively) veins with $U$ mineralization, which are hosted mainly in metarhyolites and their metapyroclastics, less in graphite-sericite, graphite and chlorite-rich phyllites (Lower Paleozoic, Gelnica Group, Gemeric Unit). The veins are located in the proximity (a maximum of several hundred meters) of the Permian Gemeric granites intrusions. 
In the area of the Majerská valley near Čučma, the following two types of these veins can be distinguished, based on their mineral composition:

(A) Quartz-fluorapatite veins containing xenotime-(Y), monazite-(Ce) monazite-(Nd), allanite-(Ce), pyrite, marcasite, uraninite, brannerite, rutile, muscovite, dravite and titanite (primary minerals); goethite, plumbogummite, goyazite, florencite-(Ce), autunite and torbernite (supergene minerals). The mentioned minerals are accompanied by younger (Alpine?) mineral associations represented by quartz II, siderite, dolomite, pyrite, arsenopyrite, pyrrhotite, marcasite, tetrahedrite, chalcopyrite, sphalerite, jamesonite, etc.

(B) Quartz veins, with only accessory amount of fluorapatite and zircon, titanite, muscovite, chlorites, rutile, leucoxenized Fe-Ti oxides and rarely U-Ti oxides. Ore mineralization is represented mainly by abundant uraninite and molybdenite. Supergene minerals are represented by bassetite, saléeite, autunite, torbernite, zeunerite, phosphuranylite, iriginite, U-rich wulfenite and other, poorly identified $\mathrm{Pb}-\mathrm{Bi}-\mathrm{U}-\mathrm{Mo}$ minerals.

The presented contribution is devoted to the results of the detailed mineralogical research of primary minerals association, and CHIME dating of uraninite, from the quartz veins with U-Mo mineralization (the above-mentioned B type), which, thus far, has been insufficiently investigated. These veins are characterized by a unique mineral association, and the mineral phases often have an unusual chemical composition or are rare also on a global scale. In addition, CHIME dating of uraninite clarifies the genetic relationship of the vein U mineralization in the Gemeric Unit to the Permian S-type granite magmatism and brings new insights into the issue of the Western Carpathians metallogeny.

\section{Regional Geological Setting}

The Gemeric Unit is one of the most important tectonic superunits in the Western Carpathians. In the north, it is thrusted along the Certovica tectonic line over the Tatric Unit; in the south, the Gemeric Unit is limited by the Lubeník-Margecany tectonic line, which separates it from the overlying Veporic Unit (Figure 1A). The Gemeric Unit forms a system of north-vergent partial nappes that are composed mainly of metamorphic pre-Carboniferous complexes and Late Paleozoic (to- Lower Triassic) syn- and post-orogenic formations [14]. It is most often divided into a northern (Klátov, Rakovec, Črmel' and Ochtiná Units) and a southern (Gelnica Group and Štós Unit) part. The Gelnica Group is represented by several thousand meters of thick Paleozoic volcanogenic (rhyolite-dacite) flysch [15,16], and its origin was associated with an active margin of Gondwana $[17,18]$. Both sedimentary and volcanic rocks have undergone regional metamorphism under the chlorite zone of the greenschist-facies conditions [19,20]. The Gelnica Group is, from the bottom to top, divided into the Vlachovo, Bystrý Potok and Drnava Formations (sensu [16,21,22]) intruded by the Permian S-type Gemeric granites [23]. According to geophysics data, these are apophyses of a larger granite body underlying metamorphosed volcano-sedimentary sequences of the Gemeric Unit [24].

All three of the following formations (sensu [16,21]) of the Gelnica Group: the Vlachovo Fm. (Lower-Middle Silurian), the Bystrý Potok Fm. (Middle Silurian) and the Lower Devonian Drnava Fm. (Figure 1B) are present within the studied area. The Bystrý potok Fm. is the most widespread, formed mainly by lydites, quartz-sericite or graphitic phyllites, metamorphosed rhyolites, dacites and their pyroclastics. Although, according to published geological maps, the Bystrý Potok Fm. is stratigraphically assigned to the Silurian [16], its magmatic rocks yielded Middle-Late Ordovician U-Th-Pb SHRIMP zircon ages of $465.8 \pm 1.5 \mathrm{Ma}[18,25]$ and 460-465 Ma, respectively [26]. According to a concept by Grecula et al. $[27,28]$, rhyolite metapyroclastics rocks (so-called porphyroids) of the Bystrý Potok Fm. are included in the Smolník Fm., and metasediments presented as eyed to gneissed metapelites, quartzites or quartzite gneisses represent the Betliar Fm. The Drnava Fm. formed by quartz, chlorite-sericite, or graphitic-sericite phyllites with acidic metapyroclastic rocks is defined by Grecula et al. $[27,28]$ as the Betliar Fm. The metacarbonates of the Vlachovo Fm. are also considered as part of the Betliar Fm. These authors present 
the stratigraphic range of both the Smolník and the Betliar Formations as Ordovician to Devonian.
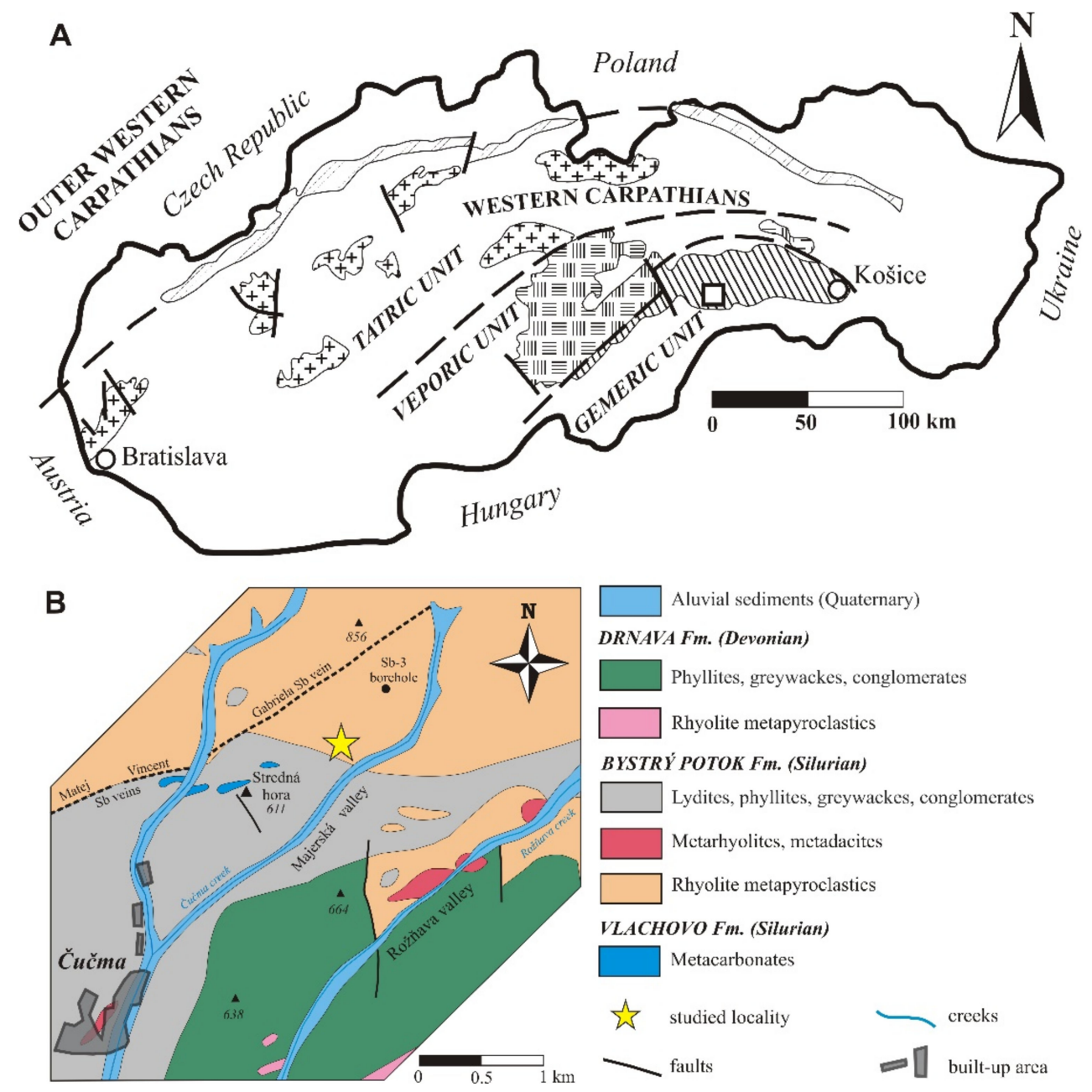

Figure 1. (A) Position of the studied locality (white square) within the main Alpine tectonic units of the Western Carpathians; (B) Geological map of the surroundings of the vein U-Mo mineralization occurrence near Čučma. According to [22].

\section{Localization and Geology of The Studied Occurrence}

The occurrence of the vein U-Mo mineralization is located in the Majerská valley near Čučma. The site is located about $7 \mathrm{~km}$ to the NNE of the district town Rožñava, $3.5 \mathrm{~km}$ to the NE of the center of the Čučma village, $950 \mathrm{~m}$ to the $\mathrm{S}$ of the elevation of $856 \mathrm{~m}$ (Lázpatak), at an altitude of about $580-630 \mathrm{~m}$. The geographical coordinates of the occurrence are E $20.568018^{\circ}$ and $\mathrm{N} 48.714321^{\circ}$.

The studied vein with U-Mo mineralization is hosted in the acidic metapyroclastics (porphyroids) of the Silurian Bystrý Potok Fm. (Figure 1B). Three veins, arranged in accordance with the metamorphic foliation of the surrounding rocks, were discovered in the area of the Majerská valley occurrence in the 1960s. The veins have a direction of $65-67^{\circ}$ to the NE, with a dip of about $36^{\circ}$ to the SE. They are 0.1 to $0.3 \mathrm{~m}$ thick and reach a length of up to $50 \mathrm{~m}$. The veins have a lenticular character, to thin out rapidly along strike and dip [1]. The mineralogy of the primary minerals of this occurrence has not yet been studied in more detail. Only quartz, apatite, uraninite and pyrite have been described within the primary vein filling [1]. 


\section{Methods Used}

Field samples were collected using an SGR scintillation radiometer (sample activity measured in cps), at a measuring range (sensitivity) of 400-3000 keV and a measurement step of $0.2 \mathrm{~s}$. Uncovered polished sections were then made from the samples for microscopic study in both transmitted and reflected light.

In situ CHIME dating of uraninite [29] was performed on a Cameca SX100 electron microanalyzer at the Dionýz Štúr State Geological Institute in Bratislava (analyzed by P. Konečný). The following conditions were used for the measurement: accelerating voltage, $15 \mathrm{kV}$; measuring current, 80-150 nA; measuring time, 75-130 s; electron beam diameter, 1-5 $\mu \mathrm{m}$. The used standards and spectral lines are as follows: $\mathrm{UO}_{2}(\mathrm{UM} \beta), \mathrm{Al}_{2} \mathrm{O}_{3}$ $(\mathrm{AlK} \alpha)$, wollastonite $(\mathrm{CaK} \alpha, \mathrm{SiK} \alpha), \mathrm{GaAs}(\mathrm{AsL} \alpha)$, apatite $(\mathrm{PK} \alpha), \mathrm{PbCO}_{3}(\mathrm{PbM} \alpha), \mathrm{ThO}_{2}$ $(\mathrm{ThM} \alpha)$, baryte $(\mathrm{SK} \alpha), \mathrm{SrTiO}_{3}(\mathrm{SrL} \alpha)$, fayalite $(\mathrm{FeK} \alpha), \mathrm{YPO}_{4}(\mathrm{YL} \alpha), \mathrm{LaPO}_{4}(\mathrm{LaL} \alpha), \mathrm{CePO}_{4}$ $(\mathrm{CeL} \alpha), \mathrm{PrPO}_{4}(\mathrm{PrL} \beta), \mathrm{NdPO}_{4}(\mathrm{NdL} \alpha), \mathrm{SmPO}_{4}(\mathrm{SmL} \alpha), \mathrm{EuPO}_{4}(\mathrm{EuL} \beta), \mathrm{GdPO}_{4}(\mathrm{GdL} \alpha)$, $\mathrm{TbPO}_{4}(\mathrm{TbL} \alpha), \mathrm{DyPO}_{4}(\mathrm{DyL} \beta), \mathrm{HoPO}_{4}(\mathrm{HoL} \beta), \mathrm{ErPO}_{4}(\mathrm{ErL} \beta), \mathrm{TmPO}_{4}(\mathrm{TmL} \alpha), \mathrm{YbPO}_{4}$ $(\mathrm{YbL} \alpha)$ and $\mathrm{LuPO}_{4}(\mathrm{LuL} \beta)$. The detection limit for the individual elements ranged from $0.01-0.1 \mathrm{wt}$. \%. Elements with content below the detection limit are not included in the tables. The following corrections were used for interferences, especially in the case of rare earth elements: $\mathrm{Pb} \times \mathrm{Y}, 0.008029 ; \mathrm{U} \times \mathrm{Th}, 0.00704 ; \mathrm{Gd} \times \mathrm{La}, 0.017352 ; \mathrm{Gd} \times \mathrm{Ce}, 0.073999$; $\mathrm{Er} \times \mathrm{Gd}, 0.027983 ; \mathrm{Lu} \times \mathrm{Yb}, 0.03688 ; \mathrm{Lu} \times \mathrm{Dy}, 0.051522 ; \mathrm{Ho} \times \mathrm{Gd}, 0.017001 ; \mathrm{Lu} \times \mathrm{Ho}$, 0.068289; Eu $\times$ Dy, 0.12; Er $\times \mathrm{Eu}, 0.022131 ;$ and $\mathrm{Tm} \times \mathrm{Sm}, 0.11517$.

The chemical composition of other minerals was determined using an Jeol-JXA-8530F electron microprobe (Institute of Earth Sciences SAS, Banská Bystrica). A microanalyzer was used for a quick determination of the chemical composition of minerals by means of an energy-dispersive spectrum (EDS), as well as for point wave dispersion microanalysis (WDS). WDS microanalyses were performed under the following conditions: accelerating voltage, $15 \mathrm{kV}$; measuring current, $20 \mathrm{nA}$ (apatite) and $20 \mathrm{kV}$; measuring current, $15 \mathrm{nA}$ (sulphides and sulphosalts). The diameter of the electron beam was in the range of 2-8 $\mu \mathrm{m}$, and ZAF correction was used. The natural/synthetic standards used and their spectral lines were as follows: $\mathrm{Ag}(\mathrm{L} \alpha)$ - $\mathrm{Ag}$, $\mathrm{Sb}(\mathrm{L} \alpha)$ —stibnite, $\mathrm{Hg}(\mathrm{M} \alpha)$-cinnabar, $\mathrm{Ni}(\mathrm{K} \alpha)$ gersdorffite, $\mathrm{Zn}(\mathrm{K} \alpha)$-sphalerite, $\mathrm{Cd}(\mathrm{L} \alpha)$ - $\mathrm{CdTe}, \mathrm{Au}(\mathrm{M} \alpha)$ - $\mathrm{Au}, \mathrm{Ca}(\mathrm{K} \alpha)$-diopside, $\mathrm{K}(\mathrm{K} \alpha)$-orthoclase, $\mathrm{U}(\mathrm{M} \beta)-\mathrm{UO}_{2}$, Th $(\mathrm{M} \alpha)$-thorianite, $\mathrm{Pb}(\mathrm{M} \beta)$-crocoite, $\mathrm{S}(\mathrm{K} \alpha)$ baryte, $\mathrm{P}(\mathrm{K} \alpha)$-apatite, $\mathrm{Y}(\mathrm{L} \alpha)-\mathrm{YPO}_{4}, \mathrm{Hf}(\mathrm{M} \alpha)-\mathrm{ZrO}_{2(\mathrm{kub} .)}, \mathrm{Zr}(\mathrm{L} \beta)-\mathrm{ZrO}_{2(\mathrm{kub} .)}, \mathrm{F}(\mathrm{K} \alpha)-$ fluorite, $\mathrm{Cl}(\mathrm{K} \alpha)$ - tugtupite, $\mathrm{Na}(\mathrm{K} \alpha)$ - albite, $\mathrm{Sr}(\mathrm{L} \alpha)$ — celestite, $\mathrm{Si}(\mathrm{K} \alpha)$ - orthoclase, $\mathrm{Al}$ $(\mathrm{K} \alpha)$-albite, $\mathrm{As}(\mathrm{L} \alpha)$ - GaAs, $\mathrm{Mg}(\mathrm{K} \alpha)$-diopside, $\mathrm{Lu}(\mathrm{L} \alpha)-\mathrm{LuPO}_{4}, \mathrm{Ho}(\mathrm{L} \beta)-\mathrm{HoPO}_{4}, \mathrm{Yb}$ $(\mathrm{L} \alpha)-\mathrm{YbPO}_{4}, \mathrm{Tm}(\mathrm{L} \alpha)-\mathrm{TmPO}_{4}, \mathrm{Er}(\mathrm{L} \alpha)-\mathrm{ErPO}_{4}, \mathrm{Gd}(\mathrm{L} \beta)-\mathrm{GdPO}_{4}, \mathrm{Dy}(\mathrm{L} \alpha)-\mathrm{DyPO}_{4}$, $\mathrm{Tb}(\mathrm{L} \alpha)-\mathrm{TbPO}_{4}, \mathrm{Sm}(\mathrm{L} \beta)-\mathrm{SmPO}_{4}, \mathrm{Eu}(\mathrm{L} \alpha)-\mathrm{EuPO}_{4}, \mathrm{Pr}(\mathrm{L} \beta)-\mathrm{PrPO}_{4}, \mathrm{Nd}(\mathrm{L} \alpha)-\mathrm{NdPO}_{4}$, $\mathrm{Ce}(\mathrm{L} \alpha)-\mathrm{CePO}_{4}, \mathrm{La}(\mathrm{L} \alpha)-\mathrm{LaPO}_{4}, \mathrm{Co}(\mathrm{K} \alpha)$ - $\mathrm{Co}, \mathrm{Zn}(\mathrm{K} \alpha)$-gahnite, $\mathrm{Cu}(\mathrm{K} \alpha)$-cuprite, $\mathrm{Fe}$ $(\mathrm{K} \alpha)$-olivine, $\mathrm{Mn}(\mathrm{K} \alpha)$-rhodonite, $\mathrm{Ti}(\mathrm{K} \alpha)$-rutile, $\mathrm{Ba}(\mathrm{L} \alpha)$-baryte, $\mathrm{Te}(\mathrm{L} \alpha)$ - CdTe, Se $(\mathrm{L} \beta)-\mathrm{Bi}_{2} \mathrm{Se}_{3}, \mathrm{Sn}(\mathrm{L} \alpha)-\mathrm{SnO}_{2}, \mathrm{~W}(\mathrm{M} \alpha)-\mathrm{CaWO}_{4}, \mathrm{Mo}(\mathrm{L} \alpha)$-wulfenite, $\mathrm{Cr}(\mathrm{K} \alpha)-\mathrm{Cr}_{2} \mathrm{O}_{3}, \mathrm{Bi}$ $(\mathrm{M} \alpha)-\mathrm{Bi}_{2} \mathrm{Se}_{3}$.

Part of quantitative chemical analyses of ore minerals were performed also on a Cameca SX100 electron-microprobe (Department of Mineralogy and Petrology, National Museum, Prague, Czech Republic), operating in the wavelength-dispersive (WDS) mode ( $25 \mathrm{kV}, 20 \mathrm{nA}$ and 2 to $5 \mu \mathrm{m}$ wide beam). The following standards and X-ray lines were used (DL-detection limit): $\mathrm{Ag}$ ( $\mathrm{Ag} L \alpha$ DL 0.07), Bi (BiM $\beta$ DL 0.28), CdTe (CdL $\alpha$ DL 0.07), chalcopyrite (CuK $\alpha$ DL 0.05, SK $\propto$ DL 0.05), Co (CoK $\alpha$ DL 0.04), halite (ClK $\alpha$ DL 0.04), HgTe (HgL $\alpha$ DL 0.27), InAs (InL $\alpha$ DL 0.05), Mn (MnK $\alpha$ DL 0.04), Ni (NiK $\alpha$ DL 0.04), NiAs (AsL $\alpha$ DL 0.06), PbS (PbM $\alpha$ DL 0.11), PbSe (SeL $\beta$ DL 0.08), pyrite (FeK $\alpha$ DL 0.04), Sb2S3 (SbL $\alpha$ DL $0.05)$, Sn (SnL $\alpha$ DL 0.05), PbTe (TeL $\alpha$ DL 0.06) and ZnS (ZnK $\alpha$ DL 0.06). The contents of the above-listed elements, which are not included in the tables, were analyzed quantitatively, but their contents were below the detection limit. Raw intensities were converted to the concentrations of elements using automatic "PAP" matrix-correction software [30].

The distribution maps of the individual elements in a typical ore material were made using X-ray fluorescence spectroscopy (XRF) at the Institute of Earth Sciences SAS in Banská 
Bystrica, using an M4 TORNADO instrument (Bruker) under the following conditions: current, $50 \mathrm{kV}$; voltage, $600 \mu \mathrm{A}$; range, $40 \mathrm{keV} / 130 \mathrm{kcps}$; and measurement time, $2 \mathrm{~h}$.

Chemical analyses of the mineralized samples were performed using the Inductively Coupled Plasma-Emission Spectrometry (ICP-ES) and Inductively Coupled Plasma-Mass Spectrometry (ICP-MS) analyses (ACME Analytical Laboratories Ltd., Vancouver, BC, Canada).

\section{Results}

\subsection{Character of Mineralization}

Quartz is a dominant gangue mineral. It has a white to grey-white color locally with a slight pinkish tint. Quartz gangue is relatively compact, and small drusy cavities are rare. Accessory minerals are represented by fluorapatite, zircon, titanite, muscovite, chlorites, tourmaline (schorl to dravite), rutile, leucoxenized Fe-Ti oxides and, rarely, U-Ti oxides. UMo mineralization (represented mainly by uraninite and molybdenite) is distributed very irregularly. Macroscopically, it forms dark smudgy accumulations, or irregular fine-grained bands or veinlets in the quartz (up to 5-6 cm in size, Figure 2). Quartz is weakly limonitized in the vicinity of accumulations of U-Mo minerals. The radioactivity of individual collected samples is reaching up to $600-700$ cps. U-Mo mineralization is often accompanied by younger, sulphidic mineralization (pyrite, sulphotellurides, sulphosalts, etc.). The chemical composition of the average mineralized gangue is illustrated in Table 1. The distribution of the individual major elements in the vein is shown in Figure 3.

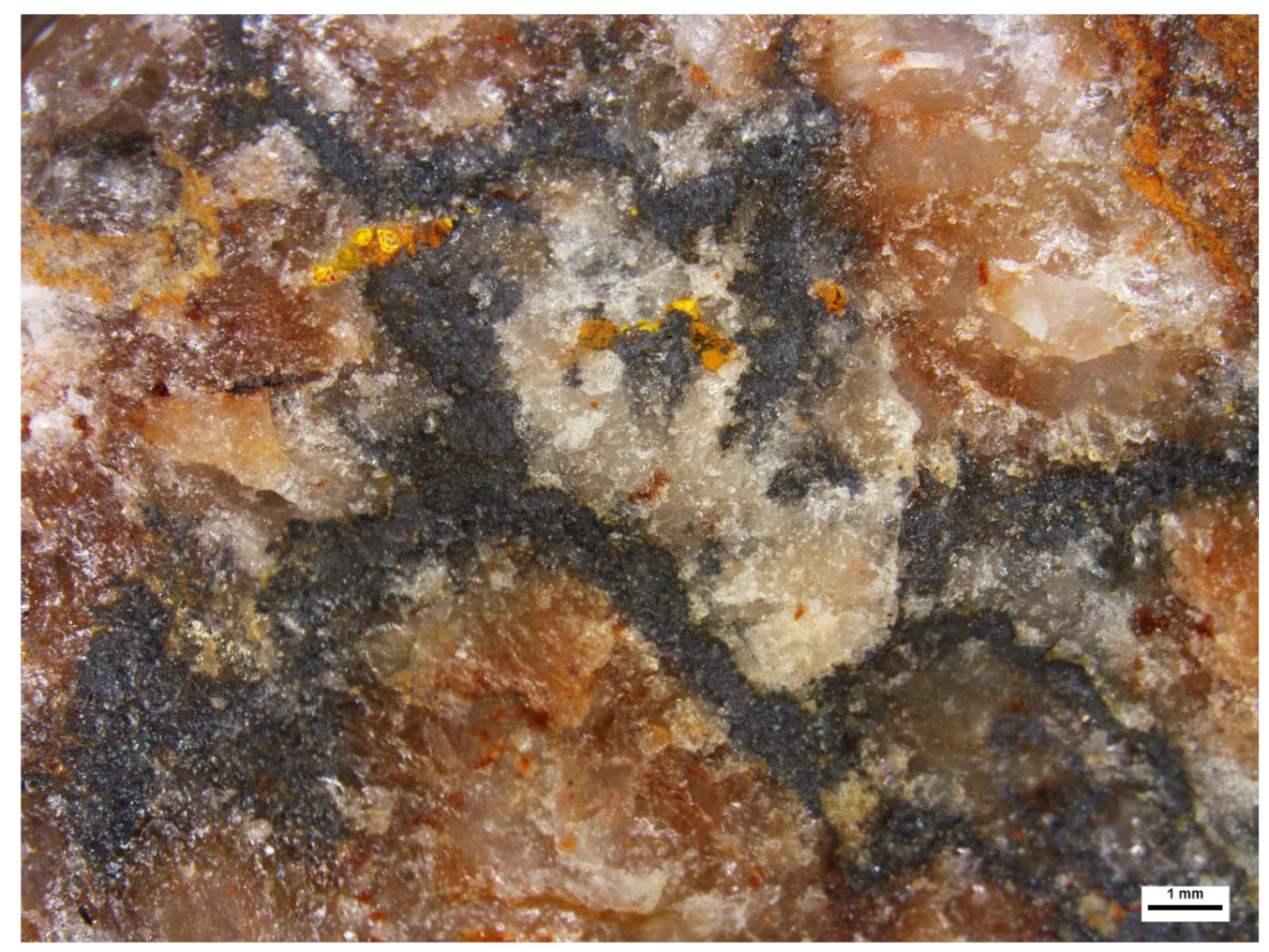

Figure 2. Irregular veinlets of U-Mo mineralization in limonitized quartz. The small cavities in the quartz are filled with a yellow uranyl molybdate-iriginite. Photo: Š. Ferenc. 
Table 1. Chemical composition of a quartz vein containing U-Mo mineralization (Q-U-Mo) in comparison to the composition of quartz-fluorapatite veins containing U-REE mineralization (Q-Ap-U-REE) from the Majerská Valley. Elements with significantly different content in both types of veins are highlighted in bold.

\begin{tabular}{|c|c|c|c|c|c|c|c|}
\hline & Q-Ap-U-REE & Q-U-Mo & & & Q-Ap-U-REE & Q-U-Mo & \\
\hline $\mathrm{Li}$ & 2.3 & 3.7 & ppm & In & 0.03 & $<0.01$ & ppm \\
\hline $\mathrm{Na}$ & 0.032 & 0.016 & wt. $\%$ & $\mathrm{Tl}$ & $<0.05$ & 0.26 & ppm \\
\hline $\mathrm{K}$ & 0.2 & 0.19 & wt. $\%$ & $\mathbf{P}$ & 3.039 & 0.031 & wt. \% \\
\hline $\mathrm{Rb}$ & 10.3 & 13.3 & ppm & As & 19.9 & 1823.9 & ppm \\
\hline Cs & 0.2 & 1.2 & ppm & $\mathrm{Sb}$ & 15.8 & 20.26 & ppm \\
\hline $\mathrm{Be}$ & $<1$ & 1 & ppm & $\mathrm{Bi}$ & 6.92 & 3.85 & ppm \\
\hline $\mathrm{Mg}$ & 0.05 & 0.1 & wt. \% & $\mathrm{Al}$ & 0.56 & 0.47 & wt. \% \\
\hline $\mathrm{Ca}$ & 7.26 & 0.08 & wt. \% & $S$ & $<0.04$ & 0.08 & wt. \% \\
\hline $\mathrm{Sr}$ & 204 & 16 & ppm & Se & $<0.3$ & $<0.3$ & ppm \\
\hline $\mathrm{Ba}$ & 73 & 39 & ppm & $\mathrm{Te}$ & 0.29 & 0.1 & ppm \\
\hline $\mathrm{Ti}$ & 0.025 & 0.011 & wt. \% & Sc & 13.7 & 5 & ppm \\
\hline $\mathrm{Zr}$ & 0.3 & 1.9 & ppm & $\mathbf{Y}$ & 104.1 & 24.5 & ppm \\
\hline Hf & $<0.02$ & $<0.02$ & ppm & La & 106.5 & 3.3 & ppm \\
\hline $\mathrm{V}$ & $<1$ & $<1$ & ppm & $\mathrm{Ce}$ & 281.8 & 8.7 & ppm \\
\hline $\mathrm{Nb}$ & 4.25 & 2.37 & ppm & Pr & 41.8 & 1.6 & ppm \\
\hline $\mathrm{Ta}$ & $<0.01$ & $<0.01$ & ppm & $\mathrm{Nd}$ & 209.5 & 9.6 & ppm \\
\hline $\mathrm{Cr}$ & 2 & 2 & ppm & Sm & 75.1 & 6.8 & ppm \\
\hline Mo & 1.51 & 73.36 & ppm & $\mathrm{Eu}$ & 29.2 & 1.5 & ppm \\
\hline W & 37 & 45.1 & ppm & Gd & 68.7 & 8.1 & ppm \\
\hline Sn & 3.4 & 0.3 & ppm & $\mathrm{Tb}$ & 10.1 & 1.7 & ppm \\
\hline $\operatorname{Re}$ & $<0.002$ & $<0.002$ & ppm & Dy & 48.5 & 10.4 & ppm \\
\hline $\mathrm{Mn}$ & 75 & 23 & ppm & Ho & 5.7 & 1.4 & ppm \\
\hline $\mathrm{Fe}$ & 0.3 & 0.45 & wt. \% & Er & 15.4 & 3.7 & ppm \\
\hline Co & 0.4 & 7.9 & ppm & $\mathrm{Tm}$ & 1.8 & 0.5 & ppm \\
\hline $\mathrm{Ni}$ & 0.1 & 6.3 & ppm & $\mathrm{Yb}$ & 13 & 3.6 & ppm \\
\hline $\mathrm{Cu}$ & 41.8 & 37.1 & ppm & $\mathrm{Lu}$ & 1.2 & 0.3 & ppm \\
\hline $\mathrm{Zn}$ & 3.4 & 9.6 & ppm & Th & 2.6 & 0.3 & ppm \\
\hline $\mathrm{Pb}$ & 189.93 & 93.23 & ppm & $\mathbf{U}$ & $>4000$ & 2639.1 & ppm \\
\hline $\mathrm{Cd}$ & 0.03 & 0.17 & ppm & YREE & 1012.4 & 85.7 & ppm \\
\hline $\mathrm{Ag}$ & 154 & 139 & ppm & LREE & 812.6 & 39.6 & ppm \\
\hline $\mathrm{Ga}$ & 3.73 & 1.55 & ppm & HREE & 95.7 & 21.6 & ppm \\
\hline
\end{tabular}

\subsection{Primary Vein Minerals}

\subsubsection{Ore (Opaque) Minerals}

Molybdenite always appears in close spatial association with uraninite, over which it usually predominates. It forms rich accumulations up to several $\mathrm{cm}$ in size, which consist of individual flaky crystals up to $20 \mu \mathrm{m}$ long (Figure 4a). Molybdenite forms rims on uraninite aggregates, respectively enclosing them (Figure $4 \mathrm{~b}$ ). The spaces between the individual molybdenite crystals are locally filled with aggregates of supergene wulfenite (Figure 4c). The chemical composition of the molybdenite from Čučma is close to the ideal formula. Only a slightly increased content of Fe (up to 0.11 wt. \%; 0.003 apfu) was determined using WDS analysis.

Uraninite is the second most widespread ore mineral after molybdenite. It forms globular aggregates up to $0.1 \mathrm{~mm}$ in size (the average size of globules is about $30-40 \mu \mathrm{m}$ ) and their clusters (also irregular aggregates), in direct association with molybdenite (Figure $4 \mathrm{a}-\mathrm{c}$ ). Locally, it also forms grains enclosed in fluorapatite. The supergene degradation of uraninite is manifested by the formation of uranyl phosphates, especially saléeite and bassetite in the close vicinity of its aggregates (Figure 4d). 

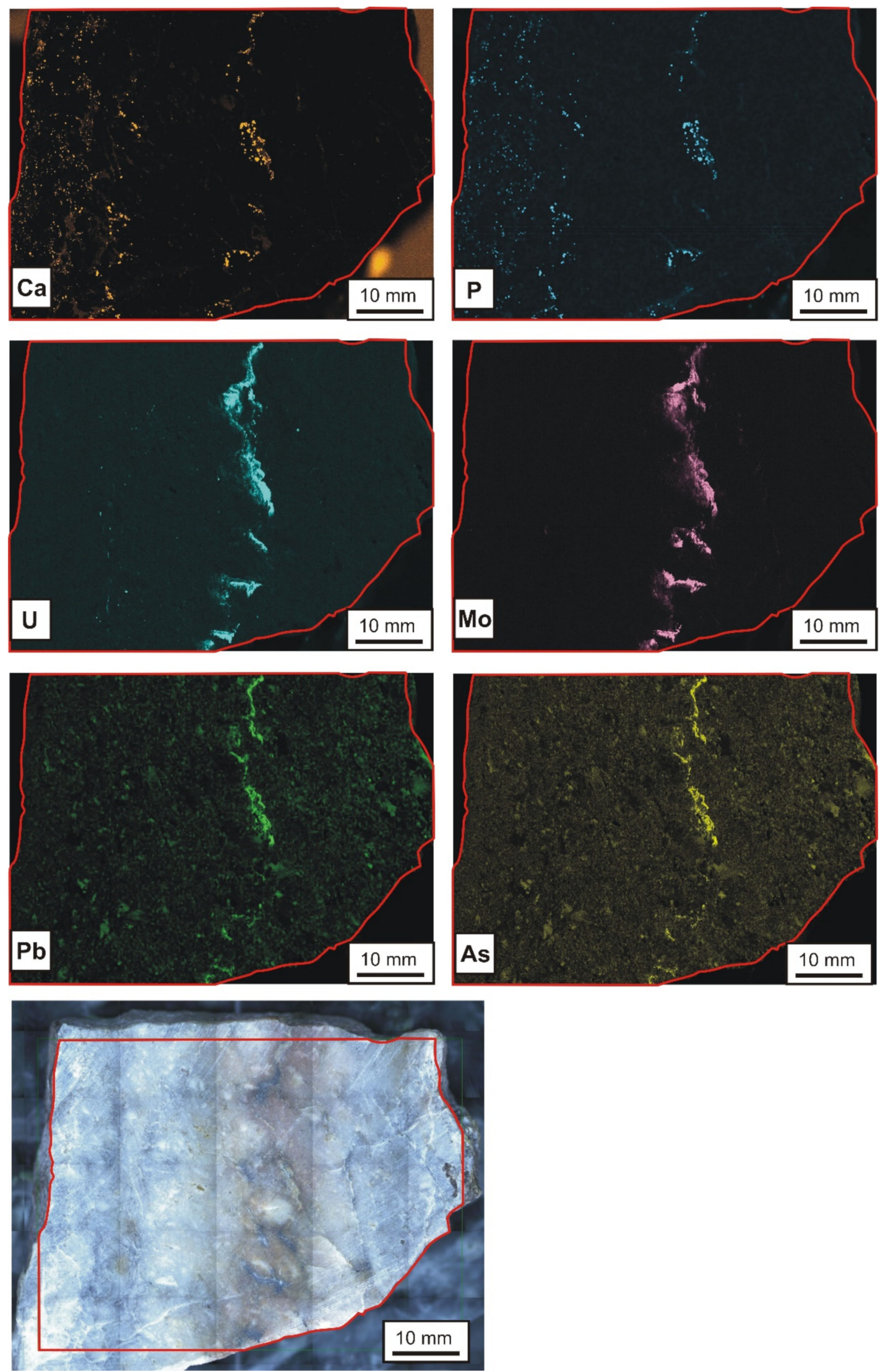

Figure 3. XRF distribution maps of selected elements in quartz vein with U-Mo mineralization (bottom subfigure). Ca and P indicate the distribution of accessory fluorapatite in quartz. Photo: S. Milovská. 

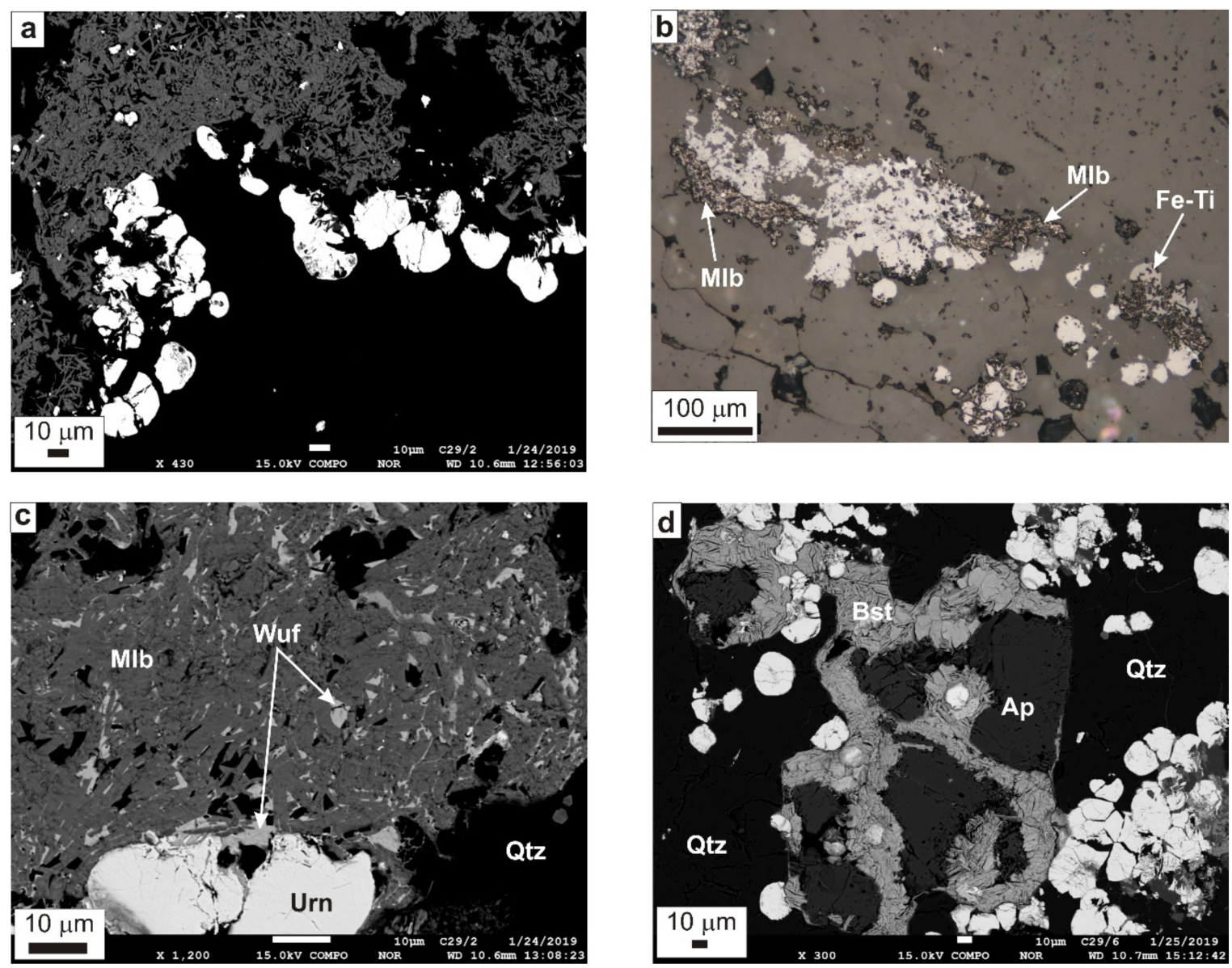

Figure 4. (a) Uraninite (white) aggregates on the border of the molybdenite vein (dark grey) and is also enclosed in molybdenite. The quartz is black. (b) Accumulation of uraninite (light grey) rimmed by fine-grained molybdenite (Mlb). At the bottom right are Fe-Ti oxides (Fe-Ti). The surroundings are quartz (dark grey). (c) Cavities and cracks in molybdenite (Mlb) aggregate are partially filled by wulfenite (Wuf), which also partially rims uraninite globules (Urn). The surrounding phase is quartz (Qtz). (d) Fluorapatite (Ap) is accompanied by uraninite (white). Supergene uranyl mineral, bassetite (Bst), encloses both minerals and also fills cracks in fluorapatite. The host mineral is quartz (Qtz). Pictures a, c, d-BSE (photo: T. Mikuš); picture b-reflected light, PPL (photo: Š. Ferenc).

The chemical analyses of the uraninite from Čučma are given in Table 2. The uranium content is relatively high and stable (0.86-0.87 apfu of $U)$, indicating that uraninite was practically unaffected by younger hydrothermal processes. Similarly, the low Si content (reaching up to 1.54 wt. \% $\mathrm{SiO}_{2} ; 0.07 \mathrm{apfu}$ of $\mathrm{Si}$ ) excludes the possibility of its later coffinitization. Thorium concentrations are very low, under the detection limit of the electron microprobe. From the other elements, the contents of $\mathrm{Pb}(2.77-3.12 \mathrm{wt}$ \% $\mathrm{PbO}$; a maximum of 0.04 apfu of $\mathrm{Pb}$ ) and $\mathrm{Ca}$ (up to 0.82 wt. \% CaO; 0.04 apfu of $\mathrm{Ca}$ ) are relatively significantly increased. $\mathrm{Y}+\mathrm{REE}$ are present in the uraninite (2.99-4.54 wt. \% $\mathrm{Y}_{2} \mathrm{O}_{3}+\mathrm{REE}_{2} \mathrm{O}_{3}$; 0.05-0.07 apfu of $Y+$ REE), while the ratio of LREE (with an average of 0.03 apfu) and HREE (with an average of $0.02 \mathrm{apfu}$ ) is relatively equal (with a moderate predominance of LREE). The content of yttrium is ranging from 0.41 to 0.77 wt. $\% \mathrm{Y}_{2} \mathrm{O}_{3}(0.03-0.04 a p f u$ of $\mathrm{Y})$. The content of other elements does not have a significant effect on the variations of the uraninite chemical composition. 
Table 2. Representative WDS microanalyses of uraninite from Čučma (Majerská valley).

\begin{tabular}{|c|c|c|c|c|c|c|c|c|c|c|c|c|c|c|}
\hline & 1 & 2 & 3 & 4 & 5 & 6 & 7 & 8 & 9 & 10 & 11 & 12 & 13 & 14 \\
\hline $\mathrm{UO}_{2}$ & 88.64 & 89.20 & 88.90 & 89.58 & 89.04 & 89.64 & 88.89 & 90.42 & 88.95 & 88.73 & 88.98 & 89.47 & 89.25 & 89.92 \\
\hline $\mathrm{CaO}$ & 0.57 & 0.54 & 0.70 & 0.67 & 0.56 & 0.63 & 0.70 & 0.67 & 0.82 & 0.75 & 0.50 & 0.69 & 0.60 & 0.67 \\
\hline $\mathrm{FeO}$ & 0.01 & 0.00 & 0.00 & 0.00 & 0.02 & 0.00 & 0.00 & 0.00 & 0.09 & 0.00 & 0.00 & 0.00 & 0.00 & 0.00 \\
\hline $\mathrm{PbO}$ & 3.08 & 3.11 & 3.08 & 3.09 & 3.08 & 3.10 & 3.07 & 3.09 & 2.77 & 3.02 & 3.08 & 3.12 & 3.04 & 3.05 \\
\hline $\mathrm{Al}_{2} \mathrm{O}_{3}$ & 0.12 & 0.08 & 0.08 & 0.08 & 0.13 & 0.10 & 0.08 & 0.11 & 0.05 & 0.12 & 0.09 & 0.07 & 0.12 & 0.05 \\
\hline $\mathrm{Y}_{2} \mathrm{O}_{3}$ & 0.42 & 0.55 & 0.61 & 0.64 & 0.34 & 0.58 & 0.64 & 0.56 & 0.77 & 0.54 & 0.59 & 0.65 & 0.41 & 0.71 \\
\hline $\mathrm{La}_{2} \mathrm{O}_{3}$ & 0.00 & 0.00 & 0.00 & 0.00 & 0.00 & 0.00 & 0.00 & 0.00 & 0.00 & 0.03 & 0.00 & 0.00 & 0.00 & 0.00 \\
\hline $\mathrm{Ce}_{2} \mathrm{O}_{3}$ & 0.23 & 0.24 & 0.24 & 0.23 & 0.31 & 0.27 & 0.21 & 0.22 & 0.34 & 0.28 & 0.34 & 0.30 & 0.25 & 0.23 \\
\hline $\mathrm{Pr}_{2} \mathrm{O}_{3}$ & 0.32 & 0.39 & 0.33 & 0.32 & 0.28 & 0.39 & 0.36 & 0.40 & 0.33 & 0.27 & 0.31 & 0.31 & 0.31 & 0.35 \\
\hline $\mathrm{Nd}_{2} \mathrm{O}_{3}$ & 0.16 & 0.17 & 0.21 & 0.18 & 0.02 & 0.15 & 0.21 & 0.19 & 0.26 & 0.16 & 0.19 & 0.23 & 0.14 & 0.22 \\
\hline $\mathrm{Sm}_{2} \mathrm{O}_{3}$ & 0.21 & 0.26 & 0.30 & 0.34 & 0.22 & 0.26 & 0.25 & 0.23 & 0.36 & 0.28 & 0.27 & 0.28 & 0.20 & 0.25 \\
\hline $\mathrm{Eu}_{2} \mathrm{O}_{3}$ & 0.30 & 0.35 & 0.30 & 0.35 & 0.30 & 0.37 & 0.31 & 0.31 & 0.36 & 0.27 & 0.31 & 0.36 & 0.35 & 0.41 \\
\hline $\mathrm{Gd}_{2} \mathrm{O}_{3}$ & 0.30 & 0.26 & 0.35 & 0.38 & 0.28 & 0.41 & 0.36 & 0.37 & 0.44 & 0.34 & 0.33 & 0.37 & 0.29 & 0.39 \\
\hline $\mathrm{Tb}_{2} \mathrm{O}_{3}$ & 0.12 & 0.09 & 0.08 & 0.14 & 0.09 & 0.09 & 0.05 & 0.09 & 0.14 & 0.10 & 0.11 & 0.09 & 0.15 & 0.17 \\
\hline $\mathrm{Dy}_{2} \mathrm{O}_{3}$ & 0.18 & 0.18 & 0.28 & 0.29 & 0.16 & 0.22 & 0.26 & 0.25 & 0.35 & 0.25 & 0.21 & 0.28 & 0.20 & 0.30 \\
\hline $\mathrm{Ho}_{2} \mathrm{O}_{3}$ & 0.09 & 0.07 & 0.03 & 0.05 & 0.03 & 0.15 & 0.10 & 0.00 & 0.03 & 0.00 & 0.07 & 0.04 & 0.09 & 0.06 \\
\hline $\mathrm{Er}_{2} \mathrm{O}_{3}$ & 0.56 & 0.70 & 0.64 & 0.59 & 0.58 & 0.65 & 0.54 & 0.57 & 0.60 & 0.63 & 0.60 & 0.61 & 0.53 & 0.59 \\
\hline $\mathrm{Tm}_{2} \mathrm{O}_{3}$ & 0.12 & 0.10 & 0.13 & 0.14 & 0.12 & 0.09 & 0.12 & 0.15 & 0.10 & 0.10 & 0.12 & 0.16 & 0.13 & 0.15 \\
\hline $\mathrm{Yb}_{2} \mathrm{O}_{3}$ & 0.26 & 0.34 & 0.30 & 0.37 & 0.23 & 0.33 & 0.32 & 0.34 & 0.36 & 0.28 & 0.33 & 0.28 & 0.28 & 0.30 \\
\hline $\mathrm{Lu}_{2} \mathrm{O}_{3}$ & 0.10 & 0.14 & 0.15 & 0.08 & 0.04 & 0.10 & 0.13 & 0.19 & 0.12 & 0.07 & 0.17 & 0.18 & 0.19 & 0.10 \\
\hline $\mathrm{SiO}_{2}$ & 1.43 & 1.05 & 1.09 & 0.95 & 1.54 & 1.05 & 0.98 & 1.30 & 0.73 & 1.39 & 1.21 & 0.92 & 1.35 & 0.77 \\
\hline $\mathrm{P}_{2} \mathrm{O}_{5}$ & 0.04 & 0.04 & 0.04 & 0.04 & 0.07 & 0.06 & 0.04 & 0.05 & 0.04 & 0.06 & 0.04 & 0.05 & 0.06 & 0.04 \\
\hline $\mathrm{As}_{2} \mathrm{O}_{5}$ & 0.16 & 0.15 & 0.15 & 0.16 & 0.18 & 0.15 & 0.16 & 0.15 & 0.16 & 0.16 & 0.15 & 0.16 & 0.16 & 0.15 \\
\hline $\mathrm{SO}_{3}$ & 0.02 & 0.01 & 0.01 & 0.02 & 0.00 & 0.00 & 0.00 & 0.03 & 0.04 & 0.00 & 0.00 & 0.01 & 0.02 & 0.02 \\
\hline Total wt. \% & 97.44 & 98.01 & 98.00 & 98.68 & 97.61 & 98.80 & 97.78 & 99.69 & 98.19 & 97.81 & 97.99 & 98.63 & 98.10 & 98.87 \\
\hline$Y+$ REE wt. \% & 3.36 & 3.82 & 3.95 & 4.09 & 2.99 & 4.06 & 3.86 & 3.86 & 4.54 & 3.59 & 3.94 & 4.16 & 3.51 & 4.21 \\
\hline \multicolumn{15}{|c|}{ empirical formula (calculation on basis of 2 oxygens) } \\
\hline $\mathrm{U}$ & 0.855 & 0.868 & 0.861 & 0.866 & 0.855 & 0.863 & 0.867 & 0.855 & 0.866 & 0.852 & 0.861 & 0.867 & 0.857 & 0.874 \\
\hline $\mathrm{Ca}$ & 0.027 & 0.026 & 0.033 & 0.031 & 0.026 & 0.029 & 0.033 & 0.030 & 0.038 & 0.035 & 0.023 & 0.032 & 0.028 & 0.031 \\
\hline $\mathrm{Fe}$ & 0.000 & 0.000 & 0.000 & 0.000 & 0.001 & 0.000 & 0.000 & 0.000 & 0.003 & 0.000 & 0.000 & 0.000 & 0 & 0.000 \\
\hline $\mathrm{Pb}$ & 0.036 & 0.037 & 0.036 & 0.036 & 0.036 & 0.036 & 0.036 & 0.035 & 0.033 & 0.035 & 0.036 & 0.037 & 0.035 & 0.036 \\
\hline $\mathrm{Al}$ & 0.006 & 0.004 & 0.004 & 0.004 & 0.006 & 0.005 & 0.004 & 0.006 & 0.002 & 0.006 & 0.005 & 0.004 & 0.006 & 0.003 \\
\hline $\mathrm{Y}$ & 0.010 & 0.013 & 0.014 & 0.015 & 0.008 & 0.013 & 0.015 & 0.013 & 0.018 & 0.012 & 0.014 & 0.015 & 0.009 & 0.016 \\
\hline $\mathrm{La}$ & 0.000 & 0.000 & 0.000 & 0.000 & 0.000 & 0.000 & 0.000 & 0.000 & 0.000 & & 0.000 & & 0.000 & 0.000 \\
\hline $\mathrm{Ce}$ & 0.004 & 0.004 & & 0.004 & & 0.004 & 0.003 & 0.003 & 0.005 & & 0.005 & & & 0.004 \\
\hline $\operatorname{Pr}$ & 0.005 & 0.006 & 0.005 & 0.005 & 0.004 & 0.006 & 0.006 & 0.006 & 0.005 & 0.004 & 0.005 & 0.005 & 0.005 & 0.006 \\
\hline $\mathrm{Nd}$ & 0.002 & 0.003 & 0.003 & 0.003 & 0.000 & 0.002 & 0.003 & 0.003 & 0.004 & 0.002 & 0.003 & 0.004 & 0.002 & 0.003 \\
\hline $\mathrm{Sm}$ & 0.003 & 0.004 & 0.004 & 0.005 & 0.003 & 0.004 & 0.004 & 0.003 & 0.005 & 0.004 & 0.004 & 0.004 & 0.003 & 0.004 \\
\hline $\mathrm{Eu}$ & 0.004 & 0.005 & 0.004 & 0.005 & 0.004 & 0.005 & 0.005 & 0.005 & 0.005 & & 0.005 & 0.005 & 0.005 & 0.006 \\
\hline $\mathrm{Gd}$ & 0.004 & 0.004 & 0.005 & 0.005 & 0.004 & 0.006 & 0.005 & 0.005 & 0.006 & 0.005 & 0.005 & 0.005 & 0.004 & 0.006 \\
\hline $\mathrm{Tb}$ & 0.002 & 0.001 & 0.001 & 0.002 & 0.001 & 0.001 & 0.001 & 0.001 & 0.002 & 0.001 & 0.002 & 0.001 & 0.002 & 0.003 \\
\hline Dy & 0.002 & 0.002 & 0.004 & 0.004 & 0.002 & 0.003 & 0.004 & 0.003 & 0.005 & 0.004 & 0.003 & 0.004 & 0.003 & 0.004 \\
\hline Ho & 0.001 & 0.001 & 0.000 & 0.001 & 0.000 & 0.002 & 0.001 & 0.000 & 0.000 & 0.000 & 0.001 & 0.001 & 0.001 & 0.001 \\
\hline Er & 0.008 & 0.010 & 0.009 & 0.008 & 0.008 & 0.009 & 0.007 & 0.008 & 0.008 & 0.008 & 0.008 & 0.008 & 0.007 & 0.008 \\
\hline $\mathrm{Tm}$ & 0.002 & 0.001 & 0.002 & 0.002 & 0.002 & 0.001 & 0.002 & 0.002 & 0.001 & 0.001 & 0.002 & 0.002 & 0.002 & 0.002 \\
\hline $\mathrm{Yb}$ & 0.003 & 0.005 & 0.004 & 0.005 & 0.003 & 0.004 & 0.004 & 0.004 & 0.005 & 0.004 & 0.004 & 0.004 & 0.004 & 0.004 \\
\hline $\mathrm{Lu}$ & 0.001 & 0.002 & 0.002 & 0.001 & 0.001 & 0.001 & 0.002 & 0.002 & 0.002 & 0.001 & 0.002 & 0.002 & 0.002 & 0.001 \\
\hline $\mathrm{Si}$ & 0.062 & 0.046 & 0.047 & 0.041 & 0.066 & 0.045 & 0.043 & 0.055 & 0.032 & 0.060 & 0.052 & 0.040 & 0.058 & 0.034 \\
\hline $\mathrm{P}$ & 0.002 & 0.002 & 0.002 & 0.001 & 0.003 & 0.002 & 0.001 & 0.002 & 0.002 & 0.002 & 0.002 & 0.002 & 0.002 & 0.001 \\
\hline As & 0.004 & 0.004 & 0.004 & 0.004 & 0.004 & 0.003 & 0.004 & 0.003 & 0.004 & 0.004 & 0.003 & 0.004 & 0.004 & 0.003 \\
\hline$S$ & 0.001 & 0.000 & 0.000 & 0.001 & 0.000 & 0.000 & 0.000 & 0.001 & 0.001 & 0.000 & 0.000 & 0.000 & 0.001 & 0.000 \\
\hline$Y+R E E$ & 0.052 & 0.060 & 0.062 & 0.065 & 0.046 & 0.064 & 0.062 & 0.059 & 0.073 & 0.056 & 0.062 & 0.066 & 0.054 & 0.067 \\
\hline LREE & 0.023 & 0.026 & 0.026 & 0.027 & 0.021 & 0.028 & 0.026 & 0.026 & 0.032 & 0.024 & 0.027 & 0.028 & 0.023 & 0.028 \\
\hline HREE & 0.019 & 0.022 & 0.022 & 0.023 & 0.017 & 0.022 & 0.021 & 0.021 & 0.023 & 0.019 & 0.022 & 0.022 & 0.021 & 0.023 \\
\hline
\end{tabular}


The chemical in situ electron-microprobe $\mathrm{U}-\mathrm{Pb}$ dating of uraninite (according to [29]) provided an age interval of between 241 and $269 \pm 2$ Ma (2 samples, 23-point analyses; Table 3). However, the majority of the age data obtained (22-point analyses) fall into the range of 261-269 $\pm 2 \mathrm{Ma}$ (with an average of $265 \pm 2 \mathrm{Ma}$ ), corresponding to the Guadalupian Epoch of Permian.

Table 3. Chemical ages of uraninite from Čučma (Majerská valley) calculated according to procedure of Montel et al. [29].

\begin{tabular}{|c|c|c|c|c|c|c|c|c|c|c|c|c|c|c|c|}
\hline & Sample & $\begin{array}{c}\mathrm{Pb} \\
\text { meas. }\end{array}$ & $\begin{array}{c}\text { Th } \\
\text { wt. } \%\end{array}$ & $\begin{array}{c}\text { U wt. } \\
\%\end{array}$ & $\begin{array}{c}\mathrm{Pb} \\
\text { wt. \% }\end{array}$ & $\begin{array}{c}\text { Y wt. } \\
\%\end{array}$ & $\mathbf{U}_{\text {corr. }}$ & $\mathrm{Pb}_{\text {corr. }}$ & $\begin{array}{l}\text { Th } \\
2 \sigma\end{array}$ & $\mathrm{U} 2 \sigma$ & $\begin{array}{l}\mathrm{Pb} \\
2 \sigma\end{array}$ & $\begin{array}{c}\text { U at. } \\
\%\end{array}$ & $\begin{array}{c}\mathrm{Pb} \text { at. } \\
\%\end{array}$ & $\begin{array}{l}\text { Age } \\
\text { (Ma) }\end{array}$ & $1 \sigma$ \\
\hline 1 & C29-6 & 2.8617 & blld & 78.1362 & 2.9151 & 0.3276 & 75.7921 & 2.9058 & 0 & 0.2715 & 0.0234 & 95.9646 & 4.0354 & 268 & 2.3 \\
\hline 2 & C29-6 & 2.8862 & blld & 78.6259 & 2.9395 & 0.4301 & 76.2671 & 2.9298 & 0 & 0.2726 & 0.0236 & 95.9568 & 4.0432 & 268 & 2.3 \\
\hline 3 & C29-6 & 2.8628 & blld & 78.3653 & 2.9156 & 0.4793 & 76.0143 & 2.9058 & 0 & 0.2718 & 0.0233 & 95.9757 & 4.0243 & 267 & 2.3 \\
\hline 4 & C29-6 & 2.8746 & blld & 78.9599 & 2.9277 & 0.5013 & 76.5911 & 2.9178 & 0 & 0.2735 & 0.0235 & 95.9892 & 4.0108 & 266 & 2.3 \\
\hline 5 & C29-6 & 2.8707 & blld & 78.8515 & 2.9238 & 0.4952 & 76.4860 & 2.9138 & 0 & 0.2731 & 0.0234 & 95.9890 & 4.0110 & 266 & 2.3 \\
\hline 6 & C29-6 & 2.8577 & blld & 78.4893 & 2.9116 & 0.2694 & 76.1346 & 2.9024 & 0 & 0.2725 & 0.0233 & 95.9870 & 4.0130 & 266 & 2.3 \\
\hline 7 & C29-6 & 2.8826 & blld & 79.0197 & 2.9360 & 0.4603 & 76.6491 & 2.9262 & 0 & 0.2735 & 0.0236 & 95.9810 & 4.0190 & 267 & 2.3 \\
\hline 8 & C29-6 & 2.8700 & blld & 78.5859 & 2.9231 & 0.4610 & 76.2283 & 2.9133 & 0 & 0.2725 & 0.0235 & 95.9767 & 4.0233 & 267 & 2.3 \\
\hline 9 & C29-6 & 2.8542 & blld & 78.3536 & 2.9069 & 0.5019 & 76.0030 & 2.8969 & 0 & 0.2720 & 0.0234 & 95.9869 & 4.0131 & 266 & 2.3 \\
\hline 10 & C29-6 & 2.8105 & blld & 78.5054 & 2.8634 & 0.4521 & 76.1502 & 2.8537 & 0 & 0.2724 & 0.0231 & 96.0530 & 3.9470 & 262 & 2.3 \\
\hline 11 & C29-2 & 2.8083 & blld & 78.4629 & 2.8613 & 0.4380 & 76.1090 & 2.8516 & 0 & 0.2723 & 0.0231 & 96.0538 & 3.9462 & 262 & 2.3 \\
\hline 12 & C29-2 & 2.8682 & blld & 79.7079 & 2.9221 & 0.4409 & 77.3167 & 2.9124 & 0 & 0.2757 & 0.0235 & 96.0334 & 3.9666 & 263 & 2.3 \\
\hline 13 & C29-2 & 2.5789 & blld & 78.4052 & 2.6304 & 0.6066 & 76.0530 & 2.6202 & 0 & 0.2719 & 0.0215 & 96.3633 & 3.6367 & 241 & 2.1 \\
\hline 14 & C29-2 & 2.8355 & blld & 78.7048 & 2.8888 & 0.4129 & 76.3437 & 2.8792 & 0 & 0.2728 & 0.0233 & 96.0285 & 3.9715 & 264 & 2.3 \\
\hline 15 & C29-2 & 2.8035 & blld & 78.2172 & 2.8563 & 0.4273 & 75.8707 & 2.8466 & 0 & 0.2717 & 0.0231 & 96.0482 & 3.9518 & 262 & 2.3 \\
\hline 16 & C29-2 & 2.8910 & blld & 78.8949 & 2.9444 & 0.4444 & 76.5281 & 2.9347 & 0 & 0.2734 & 0.0237 & 95.9637 & 4.0363 & 268 & 2.3 \\
\hline 17 & C29-2 & 2.8649 & blld & 78.4324 & 2.9179 & 0.4630 & 76.0794 & 2.9081 & 0 & 0.2722 & 0.0235 & 95.9762 & 4.0238 & 267 & 2.3 \\
\hline 18 & C29-2 & 2.8956 & blld & 78.8696 & 2.9487 & 0.5106 & 76.5035 & 2.9387 & 0 & 0.2733 & 0.0237 & 95.9565 & 4.0435 & 268 & 2.3 \\
\hline 19 & C29-2 & 2.8202 & blld & 78.6703 & 2.8739 & 0.3232 & 76.3102 & 2.8646 & 0 & 0.2729 & 0.0231 & 96.0469 & 3.9531 & 262 & 2.3 \\
\hline 20 & C29-2 & 2.8051 & blld & 78.7543 & 2.8580 & 0.4814 & 76.3917 & 2.8482 & 0 & 0.2731 & 0.0229 & 96.0724 & 3.9276 & 261 & 2.2 \\
\hline 21 & C29-2 & 2.8504 & blld & 78.3925 & 2.9031 & 0.5135 & 76.0407 & 2.8931 & 0 & 0.2721 & 0.0234 & 95.9939 & 4.0061 & 266 & 2.3 \\
\hline 22 & C29-2 & 2.8345 & blld & 79.2638 & 2.8874 & 0.5555 & 76.8859 & 2.8773 & 0 & 0.2743 & 0.0233 & 96.0579 & 3.9421 & 262 & 2.3 \\
\hline 23 & C29-2 & 2.8850 & blld & 78.4314 & 2.9380 & 0.4641 & 76.0785 & 2.9282 & 0 & 0.2721 & 0.0235 & 95.9490 & 4.0510 & 269 & 2.3 \\
\hline
\end{tabular}

C29-2: weighted average of age (263 Ma); C29-6: weighted average of age (266 Ma); C29-2 and C29-6: weighted average of age (265 Ma); blld: below lower limit of detection.

Unspecified, non-stoichiometric $\boldsymbol{U}$-Ti oxides are present only infrequently as irregular, metamict aggregates up to $0.15 \mathrm{~mm}$ in size, partially intergrown with molybdenite and uraninite (Figure 5a). Leucoxenized Fe-Ti oxides, without uranium occur in similar positions.

Pyrite is a frequent mineral. It was found in association with uraninite, molybdenite, minerals of the kobellite-tintinaite series, sulphotellurides and ullmannite. Pyrite (I) forms irregular to botryoidal aggregates up to $5 \mathrm{~mm}$ in size without chemical zoning in quartz. It grows on uraninite globules and encloses (?) grains of joséite-B. Minerals of the kobellite-tintinaite series grow on its aggregates (Figure $5 b$ ). Veinlets of pyrite II (distinct chemical zoning) cut accumulations of molybdenite and kobellite-tintinaite series minerals (Figure 5c). Pyrite is, to a great extent, intimately replaced by supergene oxide minerals (or their mixture), which is reflected in the low totals of microprobe analyses (Table 4 ) and varying ratios of cations and sulphur (0.51-0.60). The high susceptibility of pyrite to weathering processes can most likely be explained by its instability, caused by the significantly increased contents of $\mathrm{Bi}(0.01-0.07$ apfu), $\mathrm{Sb}(0.01-0.06$ apfu), $\mathrm{Pb}$ (up to $0.01 \mathrm{apfu}$ ) and $\mathrm{Cu}$ (up to $0.01 \mathrm{apfu}$ ) in the original (not weathered) mineral phase. 

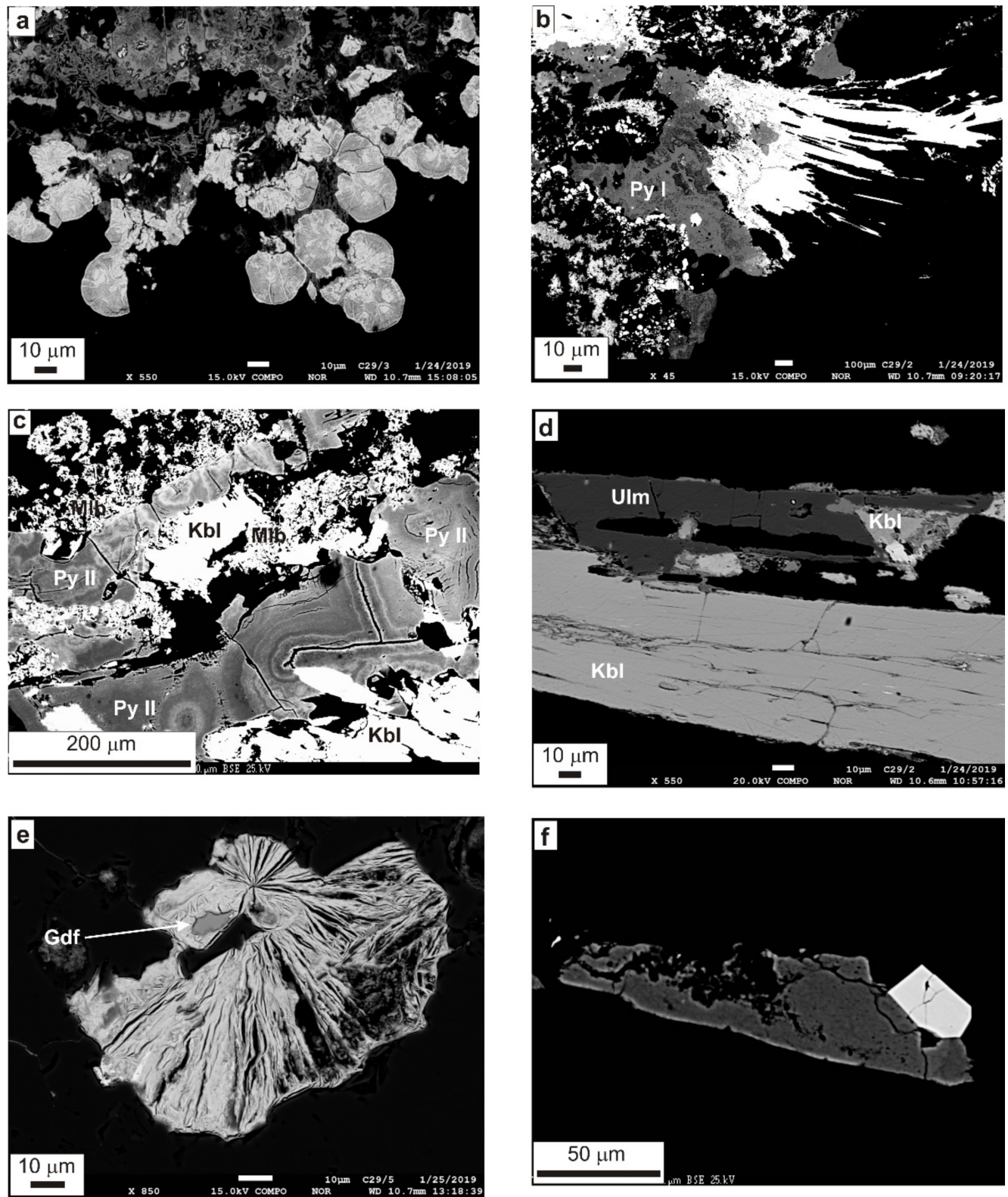

Figure 5. (a) Globular aggregates of uraninite (white) that are intensively replaced by an unidentified supergene uranyl mineral (light grey) in contact with the U-Ti oxides (tones of dark grey, top of the picture). (b) Pyrite I (Py I) overgrown by kobellite, uraninite, molybdenite, sulphotellurides and other ore minerals (white). (c) Veinlets of zonal pyrite II (Py II) cutting accumulations of molybdenite (Mlb) and kobellite-tintinaite series minerals $(\mathrm{Kbl})$. (d) Kobellite (Kbl) in contact with ullmannite (Ulm). Ullmannite is overgrown and replaced with kobellite and joséite-B (white). Quartz is black. (e) Relic of gersdorffite (Gdf) overgrown by zeunerite (light grey) in a quartz cavity (black). (f) Cobaltite (white) and pyrite (dark grey) in quartz. BSE, photos by T. Mikuš $(\mathbf{a}, \mathbf{b}, \mathbf{d}, \mathbf{e})$ and M. Števko (c,f).

Ullmannite is a rare mineral. It was found in close spatial association with kobellite in the form of elongated grains, up to $0.2 \mathrm{~mm}$ in size (Figure $5 \mathrm{~d}$ ). Ullmannite is usually replaced and overgrown by kobellite, joséite- $\mathrm{B}$ and supergene $\mathrm{Pb}$-Bi-Sb oxides. The chemical composition of ullmannite is relatively monotonous and its calculated empirical formula is close to the ideal composition of this mineral (Table 5). Only slightly increased contents of $\mathrm{Bi}$ and Se (both below $0.01 \mathrm{apfu}$ ) were detected in studied ullmannite. 
Table 4. Chemical composition of pyrite from Čučma (Majerská valley).

\begin{tabular}{cccccc}
\hline & $\mathbf{1}$ & $\mathbf{2}$ & $\mathbf{3}$ & $\mathbf{4}$ & $\mathbf{5}$ \\
\hline $\mathrm{Fe}$ & 35.36 & 37.47 & 37.72 & 40.29 & 42.34 \\
$\mathrm{Ni}$ & 0.11 & 0.09 & 0.11 & 0.07 & 0.08 \\
$\mathrm{Cu}$ & 0.27 & 0.35 & 0.34 & 0.25 & 0.33 \\
$\mathrm{~Pb}$ & 0.6 & 0.78 & 0.58 & 0.5 & 0.65 \\
$\mathrm{Sb}$ & 4.37 & 3.07 & 3.16 & 1.33 & 0.76 \\
$\mathrm{Bi}$ & 9.91 & 6.9 & 7.19 & 3.36 & 2.11 \\
$\mathrm{~S}$ & 38.59 & 42.6 & 42.31 & 47.07 & 48.51 \\
Total wt. \% & 89.21 & 91.26 & 91.41 & 92.87 & 94.78 \\
\hline \multicolumn{2}{r}{ Empirical formula (on basis of the 3 atoms) } & & & \\
\hline $\mathrm{Fe}$ & 0.985 & 0.973 & 0.981 & 0.973 & 0.990 \\
$\mathrm{Ni}$ & 0.003 & 0.002 & 0.003 & 0.002 & 0.002 \\
$\mathrm{Cu}$ & 0.007 & 0.008 & 0.008 & 0.005 & 0.007 \\
$\mathrm{~Pb}$ & 0.005 & 0.005 & 0.004 & 0.003 & 0.004 \\
$\mathrm{Sb}$ & 0.056 & 0.037 & 0.038 & 0.015 & 0.008 \\
$\mathrm{Bi}$ & 0.074 & 0.048 & 0.050 & 0.022 & 0.013 \\
\hline$\Sigma$ & 1.128 & 1.073 & 1.083 & 1.020 & 1.024 \\
\hline $\mathrm{S}$ & 1.872 & 1.927 & 1.917 & 1.980 & 1.976 \\
$\mathrm{Cat./S}$ & 0.60 & 0.56 & 0.57 & 0.51 & 0.52 \\
\hline
\end{tabular}

Table 5. Representative WDS microanalyses of ullmannite from Čučma (Majerská valley).

\begin{tabular}{cccc}
\hline & $\mathbf{1}$ & $\mathbf{2}$ & $\mathbf{3}$ \\
\hline $\mathrm{Ni}$ & 27.21 & 27.43 & 27.46 \\
$\mathrm{Co}$ & 0.05 & 0.09 & 0.03 \\
$\mathrm{Fe}$ & 0.04 & 0.03 & 0.04 \\
$\mathrm{As}$ & 0.00 & 0.07 & 0.01 \\
$\mathrm{Sb}$ & 56.20 & 56.00 & 55.92 \\
$\mathrm{Bi}$ & 0.12 & 0.08 & 0.17 \\
$\mathrm{Se}$ & 0.05 & 0.03 & 0.12 \\
$\mathrm{~S}$ & 14.42 & 14.48 & 14.85 \\
Total wt. \% & 98.09 & 98.21 & 98.59 \\
\hline & Empirical formula (on basis of 3 atoms) \\
\hline $\mathrm{Ni}$ & 1.009 & 1.014 & 1.007 \\
$\mathrm{Co}$ & 0.002 & 0.003 & 0.001 \\
$\mathrm{Fe}$ & 0.001 & 0.001 & 0.002 \\
$\mathrm{As}$ & 0.000 & 0.002 & 0.000 \\
$\mathrm{Sb}$ & 1.005 & 0.998 & 0.988 \\
$\mathrm{Bi}$ & 0.001 & 0.001 & 0.002 \\
$\mathrm{Se}$ & 0.001 & 0.001 & 0.003 \\
$\mathrm{~S}$ & 0.980 & 0.980 & 0.997 \\
\hline
\end{tabular}

Gersdorffite was found only in one case. It forms an irregular grain, about $10 \mu \mathrm{m}$ in size, overgrown with the supergene uranyl arsenate - zeunerite (Figure 5e), in quartz cavity. The chemical composition of gersdorffite was verified only by non-standardized EDS analysis.

Cobaltite occurs only rarely as individual, euhedral to subhedral crystals up to $30 \mu \mathrm{m}$ in size, either enclosed in quartz or growing over pyrite (Figure 5f). The quantitative chemical analyses of cobaltite are shown in Table 6. The two compositional varieties of cobaltite were distinguished (Figure 6) with the following average empirical formulae: $\left(\mathrm{Co}_{0.57} \mathrm{Ni}_{0.22} \mathrm{Fe}_{0.20}\right)_{\Sigma 0.99} \mathrm{As}_{0.98} \mathrm{~S}_{1.02}$ and $\left(\mathrm{Co}_{0.80} \mathrm{Fe}_{0.14} \mathrm{Ni}_{0.06}\right)_{\Sigma 1.00} \mathrm{As}_{0.90} \mathrm{~S}_{1.10}$. 
Table 6. Representative WDS microanalyses of cobaltite from Čučma (Majerská valley).

\begin{tabular}{|c|c|c|c|c|c|c|c|c|}
\hline & 1 & 2 & 3 & 4 & 5 & 6 & 7 & 8 \\
\hline $\mathrm{Fe}$ & 4.94 & 4.48 & 4.48 & 4.30 & 5.63 & 7.12 & 6.46 & 6.57 \\
\hline Co & 28.10 & 27.69 & 29.70 & 29.39 & 29.43 & 18.95 & 20.86 & 21.21 \\
\hline $\mathrm{Ni}$ & 2.19 & 2.90 & 2.20 & 2.33 & 1.21 & 8.80 & 7.55 & 7.26 \\
\hline As & 41.96 & 42.17 & 41.43 & 41.81 & 40.13 & 44.60 & 44.33 & 44.32 \\
\hline$S$ & 21.85 & 21.22 & 21.38 & 21.25 & 22.28 & 19.63 & 19.61 & 19.78 \\
\hline Total wt. \% & 99.05 & 98.46 & 99.19 & 99.08 & 98.68 & 99.10 & 98.81 & 99.14 \\
\hline \multicolumn{9}{|c|}{ Empirical formula (on basis of the 3 atoms) } \\
\hline $\mathrm{Fe}$ & 0.144 & 0.132 & 0.131 & 0.126 & 0.163 & 0.212 & 0.193 & 0.195 \\
\hline Co & 0.776 & 0.773 & 0.821 & 0.815 & 0.809 & 0.534 & 0.589 & 0.597 \\
\hline $\mathrm{Ni}$ & 0.061 & 0.081 & 0.061 & 0.065 & 0.033 & 0.249 & 0.214 & 0.205 \\
\hline$\Sigma$ & 0.980 & 0.986 & 1.013 & 1.005 & 1.006 & 0.995 & 0.996 & 0.997 \\
\hline As & 0.911 & 0.926 & 0.901 & 0.912 & 0.868 & 0.989 & 0.985 & 0.981 \\
\hline$S$ & 1.109 & 1.089 & 1.086 & 1.083 & 1.126 & 1.017 & 1.019 & 1.023 \\
\hline$\Sigma$ & 2.020 & 2.014 & 1.987 & 1.995 & 1.994 & 2.005 & 2.004 & 2.003 \\
\hline
\end{tabular}

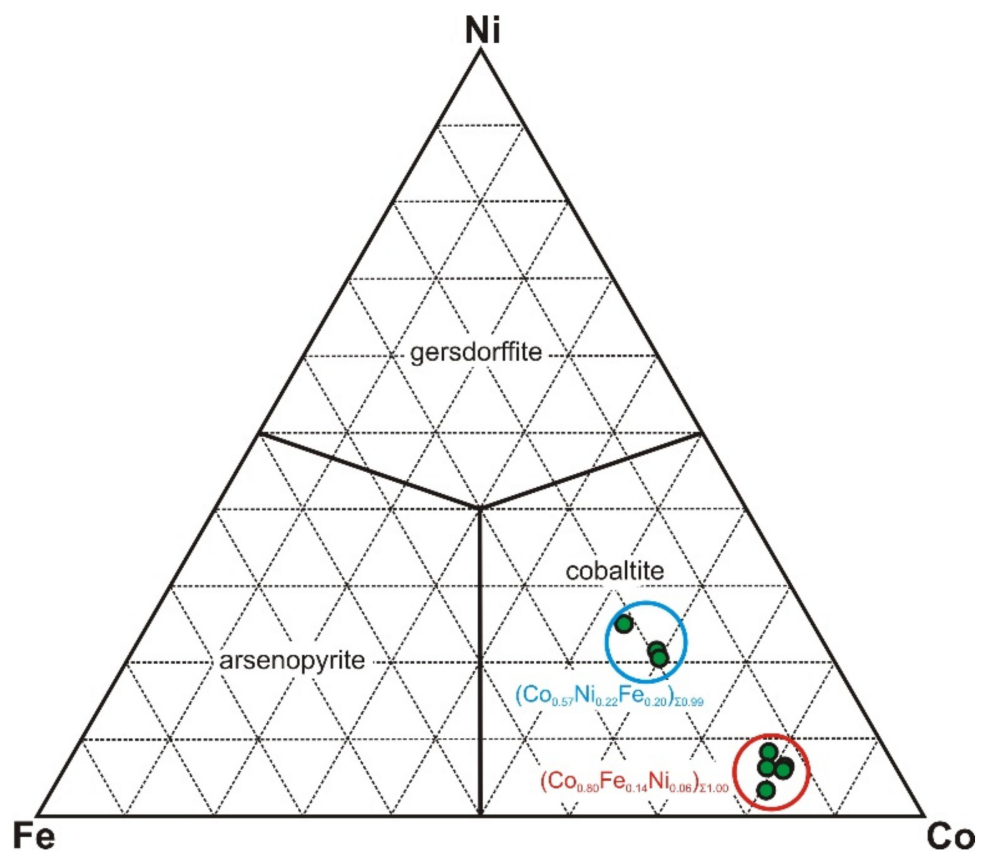

Figure 6. Chemical composition of cobaltite from Čučma in ternary system Fe-Co-Ni.

Tetradymite was found in only one case. It forms an irregular grain (0.3 $\mathrm{mm}$ in size) in close spatial association with molybdenite, uraninite, titanite and chlorite (Figure 7a). The chemical composition of tetradymite is relatively stable (Table 7, Figure 8), containing only minor amounts of $\mathrm{Sb}$ (about 0.06 apfu), $\mathrm{Pb}$ (up to 0.01 apfu) and Se (with a maximum of $0.03 a p f u$ ). The average empirical formula of the tetradymite from Čučma based on five atoms is $\left(\mathrm{Bi}_{2.03} \mathrm{Sb}_{0.06} \mathrm{~Pb}_{0.01}\right)_{\Sigma 2.10} \mathrm{Te}_{1.92}\left(\mathrm{~S}_{0.96} \mathrm{Se}_{0.02}\right)_{\Sigma 0.98}$. 

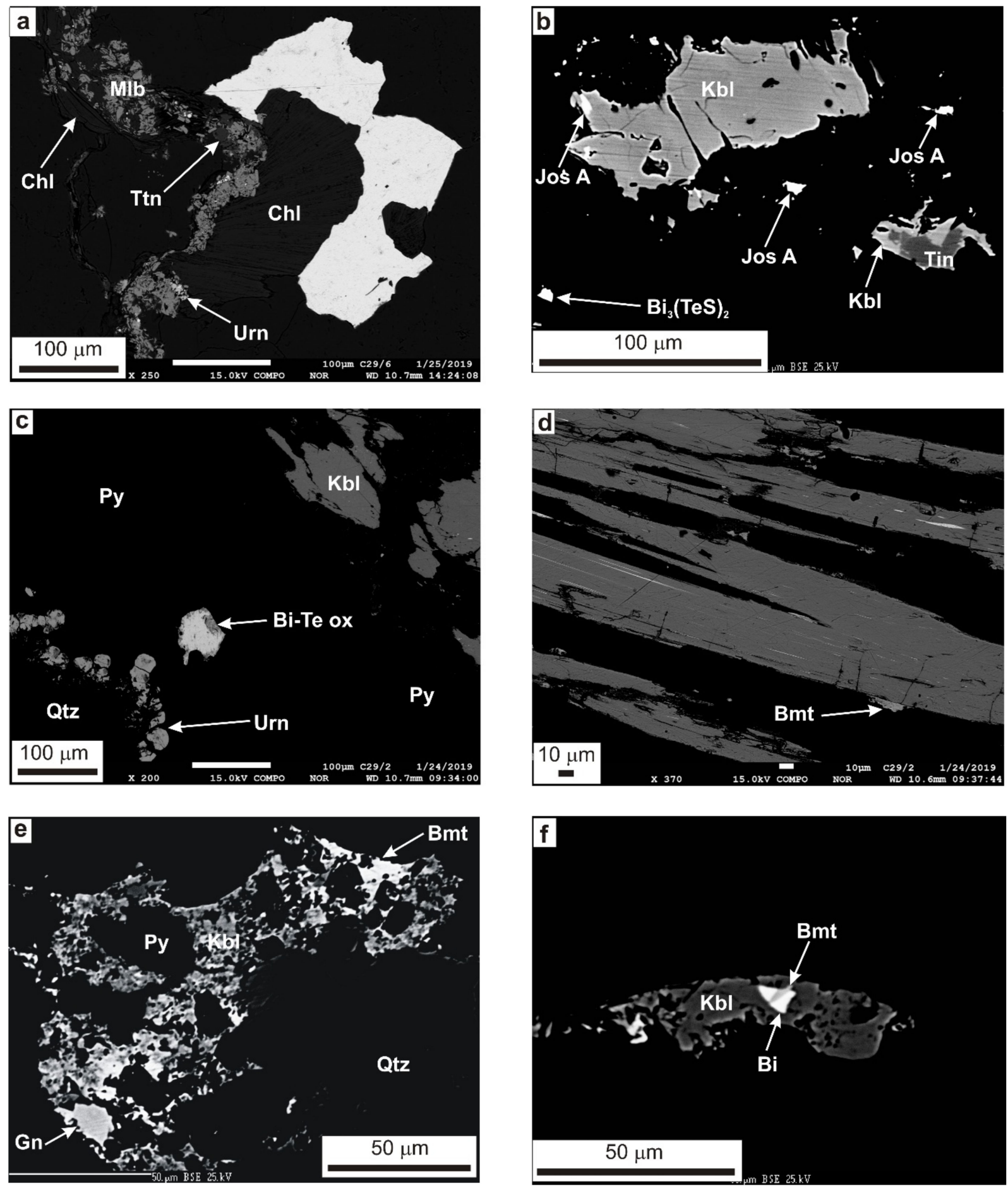

Figure 7. (a) Tetradymite aggregate (white) in spatial association with chlorite (Chl), titanite (Ttn), molybdenite (Mlb) and uraninite (Urn). The host mineral is quartz (black). (b) Aggregates of kobellite-tintinaite series minerals (Kbl and Tin, respectively) accompanied by joséite- $\mathrm{A}$ ( $\mathrm{Jos} \mathrm{A}$ ) and $\mathrm{Bi}_{3}(\mathrm{TeS})_{2}$ mineral phase ("protojoséite") in quartz (black). (c) Grain of joséite-B (white), rimmed by Bi-Te oxides (Bi-Te ox) in pyrite I (Py). In the top right are grains of minerals of the kobellite-tintinaite series $(\mathrm{Kbl})$, and in the bottom left is a pyrite aggregate lined with uraninite (Urn). To the left of uraninite is quartz (Qtz). (d) Needle-like crystals of kobellite-tintinaite (dark grey) with lamellae of the nameless $\mathrm{Bi}_{3}(\mathrm{Te}, \mathrm{S})_{2}$ mineral phase (white) corresponding to the "protojoséite". Bismuthinite (Bmt) is grown on kobellite-tintinaite crystals. (e) Pyrite (Py) and galena (Gn) are enclosed by kobellite-tintinaite series minerals (Kbl), which overgrows with bismuthinite (Bmt) in quartz (Qtz). (f) Bismuthinite (Bmt) overgrown by bismuth (Bi); both are enclosed in kobellite-tintinaite series minerals. Host mineral is quartz (black). BSE, photos by T. Mikuš (a,c,d) and M. Števko (b,e,f). 
Table 7. Chemical composition of tetradymite (an. 1-3), joséite-A (an. 4-8), joséite-B (an. 9-11) and "protojoséite", Bi 3 (TeS) 2 , (an. 12-14) from Čučma (Majerská valley).

\begin{tabular}{|c|c|c|c|c|c|c|c|c|c|c|c|c|c|c|}
\hline & 1 & 2 & 3 & 4 & 5 & 6 & 7 & 8 & 9 & 10 & 11 & 12 & 13 & 14 \\
\hline $\mathrm{Bi}$ & 59.64 & 59.76 & 59.07 & 77.84 & 78.66 & 78.73 & 79.51 & 79.56 & 75.01 & 74.34 & 75.10 & 71.06 & 72.41 & 72.27 \\
\hline $\mathrm{Sb}$ & 0.82 & 1.04 & 1.01 & 0.53 & 0.29 & 0.29 & 0.29 & 0.29 & 0.40 & 0.43 & 0.36 & 1.96 & 0.55 & 0.65 \\
\hline $\mathrm{Fe}$ & 0.00 & 0.01 & 0.00 & 0.46 & 0.70 & 0.24 & 0.05 & 0.06 & 0.19 & 0.28 & 0.22 & 0.09 & 0.00 & 0.00 \\
\hline $\mathrm{Cu}$ & 0.00 & 0.00 & 0.00 & 0.08 & 0.00 & 0.00 & 0.00 & 0.07 & 0.00 & 0.00 & 0.00 & 0.23 & 0.44 & 0.47 \\
\hline In & & & & 0.06 & 0.00 & 0.09 & 0.08 & 0.00 & & & & & 0.00 & 0.06 \\
\hline $\mathrm{Pb}$ & 0.00 & 0.19 & 0.32 & 1.60 & 1.17 & 0.93 & 1.44 & 1.45 & 0.98 & 1.04 & 1.38 & 2.28 & 2.84 & 2.80 \\
\hline $\mathrm{Te}$ & 34.14 & 34.03 & 34.63 & 11.74 & 11.96 & 11.99 & 12.18 & 11.95 & 21.56 & 21.75 & 21.49 & 17.86 & 17.89 & 17.31 \\
\hline$S$ & 4.22 & 4.44 & 4.34 & 6.13 & 5.96 & 6.58 & 6.07 & 6.12 & 2.63 & 2.68 & 2.75 & 4.32 & 3.44 & 3.70 \\
\hline Se & 0.26 & 0.20 & 0.30 & 1.00 & 0.91 & 1.09 & 0.90 & 0.85 & 0.09 & 0.20 & 0.19 & 1.07 & 1.13 & 1.05 \\
\hline Total wt. \% & 99.08 & 99.67 & 99.66 & 99.44 & 99.65 & 99.94 & 100.52 & 100.35 & 100.87 & 100.71 & 101.50 & 98.85 & 98.70 & 98.31 \\
\hline \multicolumn{15}{|c|}{ Empirical formulae } \\
\hline $\mathrm{Bi}$ & 2.054 & 2.033 & 2.011 & 3.776 & 3.829 & 3.758 & 3.873 & 3.876 & 4.036 & 3.980 & 3.996 & 2.575 & 2.734 & 2.715 \\
\hline $\mathrm{Sb}$ & 0.048 & 0.061 & 0.059 & 0.044 & 0.024 & 0.024 & 0.024 & 0.024 & 0.037 & 0.040 & 0.033 & 0.122 & 0.035 & 0.042 \\
\hline $\mathrm{Fe}$ & 0.000 & 0.001 & 0.000 & 0.084 & 0.128 & 0.043 & 0.009 & 0.011 & 0.039 & 0.055 & 0.045 & 0.012 & 0.000 & 0.000 \\
\hline $\mathrm{Cu}$ & 0.000 & 0.000 & 0.000 & 0.013 & 0.000 & 0.000 & 0.000 & 0.011 & 0.000 & 0.000 & 0.000 & 0.027 & 0.055 & 0.058 \\
\hline In & & & & 0.005 & 0.000 & 0.008 & 0.007 & 0.000 & & & & & 0.000 & 0.004 \\
\hline $\mathrm{Pb}$ & 0.000 & 0.007 & 0.011 & 0.078 & 0.057 & 0.045 & 0.071 & 0.071 & 0.053 & 0.056 & 0.074 & 0.083 & 0.108 & 0.106 \\
\hline$\Sigma$ & 2.103 & 2.102 & 2.081 & 4.000 & 4.038 & 3.877 & 3.985 & 3.994 & 4.165 & 4.131 & 4.148 & 2.819 & 2.933 & 2.925 \\
\hline $\mathrm{Te}$ & 1.926 & 1.896 & 1.930 & 0.933 & 0.953 & 0.937 & 0.972 & 0.953 & 1.900 & 1.907 & 1.873 & 1.060 & 1.106 & 1.065 \\
\hline S & 948 & 0.984 & 0.962 & 1.939 & 1.891 & 2.047 & 1.928 & 1.943 & 0.922 & 0.934 & 0.952 & 1.019 & 0.848 & 0.906 \\
\hline Se & 0.024 & 0.018 & 0.027 & 0.128 & 0.117 & 0.138 & 0.116 & 0.110 & 0.013 & 0.028 & 0.026 & 0.103 & 0.113 & 0.104 \\
\hline$\Sigma$ & 0.972 & 1.002 & 0.988 & 2.067 & 2.008 & 2.185 & 2.044 & 2.053 & 0.935 & 0.962 & 0.979 & 1.122 & 0.961 & 1.010 \\
\hline
\end{tabular}

Empirical formulae were calculated on the basis of 5 atoms (tetradymite, Bi3(TeS)2 phase) and 7 atoms (joséite A, B).

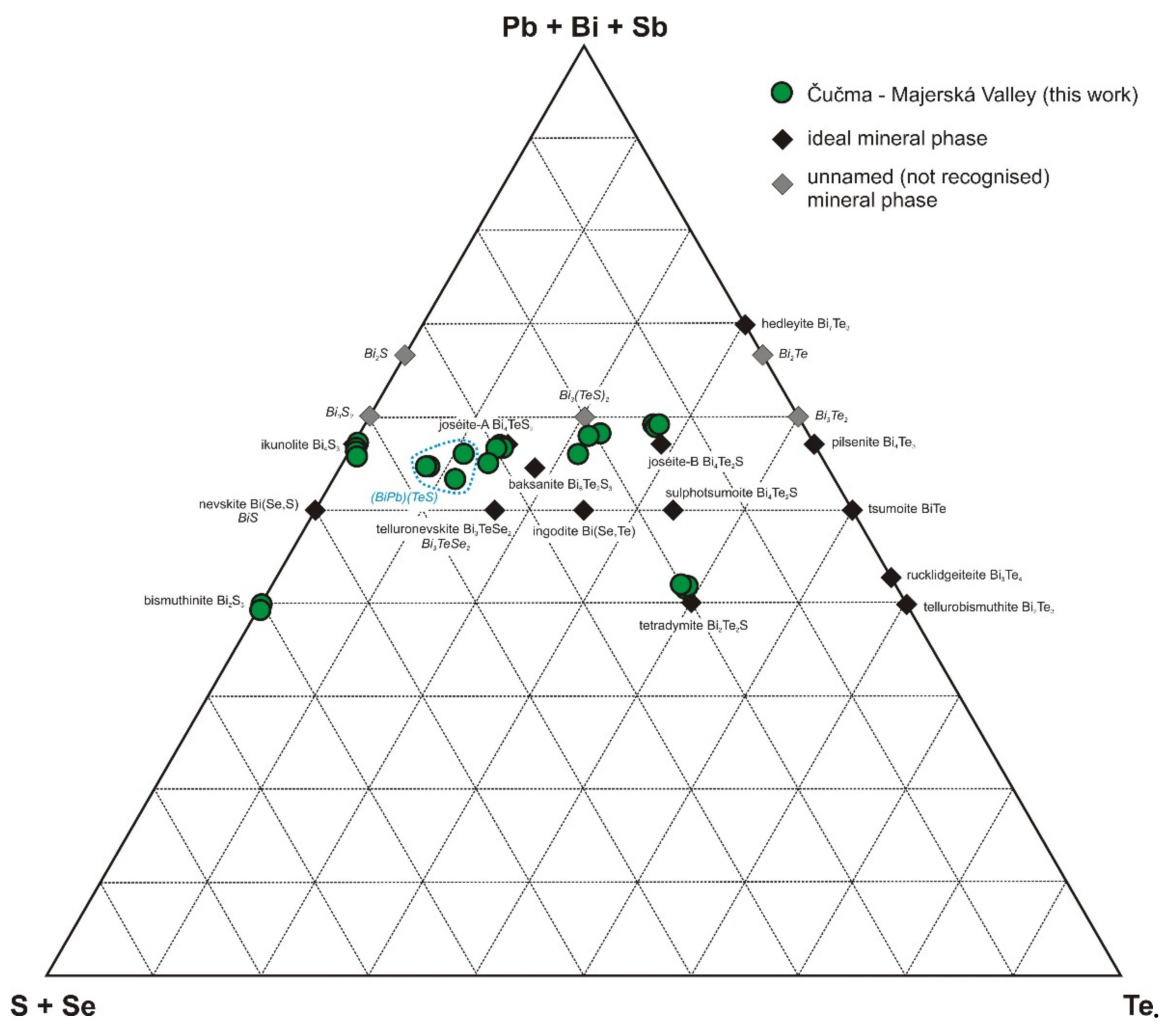

Figure 8. Chemical composition of the sulphotellurides, ikunolite and bismuthinite from Čučma in ternary system $\mathrm{S}+\mathrm{Se}-\mathrm{Te}-\mathrm{Pb}+\mathrm{Bi}+\mathrm{Sb}$. 
Joséite- $\boldsymbol{A}$ is relatively common as anhedral grains up to $10 \mu \mathrm{m}$, enclosed in pyrite together with minerals of kobellite-tintinaite series (Figure $7 \mathrm{~b}$ ). The chemical composition of the studied joséite-A is close to the ideal composition of this mineral phase (Table 7, Figure 8). The most important admixture in the joséite-A from Čučma is the content of $\mathrm{Pb}$, ranging from 0.93 to $1.60 \mathrm{wt}$. \% (0.05-0.08 apfu). Minor amounts of antimony (on average, 0.34 wt. \%; $0.03 \mathrm{apfu}$ ) and iron (up to $0.70 \mathrm{wt}$. \%; $0.13 \mathrm{apfu}$ ) were also detected. The anionic position is characterized by the stable presence of Se (with an average of 0.95 wt. \%; $0.12 \mathrm{apfu}$. The average chemical composition of the studied joséite-A is $\left(\mathrm{Bi}_{3.82} \mathrm{~Pb}_{0.06} \mathrm{Fe}_{0.05} \mathrm{Sb}_{0.03}\right)_{\Sigma 3.96} \mathrm{Te}_{0.95}\left(\mathrm{~S}_{1.95} \mathrm{Se}_{0.12}\right)_{\Sigma 2.07}$.

Joséite- $\boldsymbol{B}$ was rarely found in association with uraninite, pyrite and minerals of the kobellite-tintinaite series (Figure 7c). It forms individual grains (up to $0.05 \mathrm{~mm}$ in size) in pyrite, rimmed by an unspecified supergene Bi-Te oxidic mineral phase. The studied mineral phase has a relatively monotonous chemical composition, which is close to the ideal formula, $\mathrm{Bi}_{4} \mathrm{Te}_{2} \mathrm{~S}$ (Table 7, Figure 8). Only minor contents of $\mathrm{Sb}$ (with an average of 0.40 wt. \%; $0.04 a p f u$ ), $\mathrm{Pb}$ (with an average of 1.13 wt. \%; $0.06 \mathrm{apfu}$ ) and $\mathrm{Fe}$ (up to $0.23 \mathrm{wt}$ \%; $0.05 \mathrm{apfu}$ ) were observed in the joséite-B from Čučma. In the anionic position, $\mathrm{S}$ is replaced by a minor content of Se (up to $0.20 \mathrm{wt}$. \%; $0.03 \mathrm{apfu}$ ). The average chemical composition of the studied joséite- $\mathrm{B}$ can be expressed as $\left(\mathrm{Bi}_{4.00} \mathrm{~Pb}_{0.06} \mathrm{Fe}_{0.05} \mathrm{Sb}_{0.04}\right)_{\Sigma 4.15} \mathrm{Te}_{1.89}\left(\mathrm{~S}_{0.94} \mathrm{Se}_{0.02}\right)_{\Sigma 0.96}$.

An Unnamed $\mathrm{Bi}_{3}(\mathrm{Te}, \mathrm{S})_{2}$ mineral phase forms a thin lamellae enclosed in minerals of the kobellite-tintinaite series (Figure 7d), or small irregular grains in quartz (Figure 7b). Its chemical composition corresponds to the transitional phase between joséite-A and joséite-B and approaches the discredited protojoséite (Table 7, Figure 8). Compared to joséite-A and $\mathrm{B}$, it has a more significantly increased $\mathrm{Pb}$ content (on average, $2.64 \mathrm{wt}$. \%; $0.1 \mathrm{apfu}$ ) and a slightly increased Sb content (with an average of $1.05 \mathrm{wt}$. \%; $0.07 \mathrm{apfu}$ ). The average $\mathrm{Cu}$ content is $0.38 \mathrm{wt}$. \% (0.05 apfu). The Te content is around $17.68 \mathrm{wt}$ \% \% (1.08 apfu). The S content is, on average, $3.82 \mathrm{wt}$ \% (0.92 apfu) and the Se content is $1.08 \mathrm{wt}$ \% (0.11 apfu). The $\mathrm{Te} /(\mathrm{S}+\mathrm{Se})$ contents ratio is nearly equal (1.05). The chemical composition of this mineral phase can be expressed as $\left(\mathrm{Bi}_{2.67} \mathrm{~Pb}_{0.10} \mathrm{Sb}_{0.07} \mathrm{Cu}_{0.05}\right)_{\Sigma 2.89}\left(\mathrm{Te}_{1.08} \mathrm{~S}_{0.92} \mathrm{Se}_{0.11}\right)_{\Sigma 2.11}$.

The Unnamed (BiPb)(TeS) phase is very rare as tiny inclusions (reaching up to $10 \mu \mathrm{m}$ in size) enclosed in minerals of the kobellite-tintinaite series. This mineral phase is characterized by variable chemical composition (Table 8 , Figure 8 ). The content of Bi varies in the range of $64.37-71.68 \mathrm{wt}$. \% (0.82-0.9 apfu), the content of Sb (1.62-4.13 wt. \%; 0.04-0.09 apfu) and $\mathrm{Pb}$ (6.33-11.40 wt. \%; 0.09-0.15 apfu) also changes more significantly. A small amount of $\mathrm{Cu}$ is stably present (with an average of $0.38 \mathrm{wt}$. \%; $0.02 \mathrm{apfu}$ ). The content of Te ranges from 7.59 to $10.71 \mathrm{wt}$ \% (0.16-0.22 apfu), the $\mathrm{S}$ content is in the range of $6.63-8.56$ wt. \% (0.60-0.70 apfu) and the average Se content is 1.40 wt. \% (0.05 apfu). $\mathrm{The}(\mathrm{Bi}+\mathrm{Sb}+\mathrm{Pb}) /(\mathrm{Te}+\mathrm{S}+\mathrm{Se})$ contents ratio is around 1.23. Based on the molar ratios of the elements, the following empirical formula can be proposed for this mineral phase: $\left(\mathrm{Bi}_{0.90} \mathrm{~Pb}_{0.11} \mathrm{Sb}_{0.08} \mathrm{Cu}_{0.02}\right)_{\Sigma 1.11}\left(\mathrm{Te}_{0.19} \mathrm{~S}_{0.66} \mathrm{Se}_{0.05}\right)_{\Sigma 0.90}$.

Ikunolite is rare and it forms anhedral grains (up to $10 \mu \mathrm{m}$ in size) enclosed together with kobellite in aggregates of pyrite. The studied ikunolite has a homogenous chemical composition (Table 9, Figure 8), which almost coincides with the ideal formula, $\mathrm{Bi}_{4} \mathrm{~S}_{3}$. In the cationic position, only an insignificantly increased $\mathrm{Cu}$ content (up to $0.21 \mathrm{wt}$. \%; $0.03 \mathrm{apfu}$ ) was detected. The content of other elements is negligible. The anionic position is characterized by the dominance of $S$ (with an average of $10.39 \mathrm{wt}$. \%; $3.01 \mathrm{apfu}$ ). A minor content of Te is present (up to $0.96 \mathrm{wt}$. \%; $0.07 \mathrm{apfu}$ ) and the Se content reaches only up to $0.19 \mathrm{wt}$. \% (0.02 apfu). The average empirical formula of the ikunolite from Čučma is $\left(\mathrm{Bi}_{3.93} \mathrm{Cu}_{0.01}\right)_{\Sigma 3.94}\left(\mathrm{~S}_{3.01} \mathrm{Te}_{0.03} \mathrm{Se}_{0.01}\right)_{\Sigma 3.05}$. 
Table 8. Representative WDS microanalyses of unnamed $(\mathrm{BiPb})(\mathrm{TeS})$ mineral phase from Čučma (Majerská valley).

\begin{tabular}{ccccc}
\hline & $\mathbf{1}$ & $\mathbf{2}$ & $\mathbf{3}$ & $\mathbf{4}$ \\
\hline $\mathrm{Bi}$ & 64.37 & 70.13 & 71.34 & 71.68 \\
$\mathrm{Sb}$ & 4.13 & 1.62 & 3.94 & 4.01 \\
$\mathrm{Fe}$ & 0.17 & 0.17 & 0.00 & 0.00 \\
$\mathrm{Cu}$ & 0.46 & 0.28 & 0.37 & 0.40 \\
$\mathrm{In}$ & 0.00 & 0.07 & 0.00 & 0.07 \\
$\mathrm{~Pb}$ & 11.40 & 6.33 & 7.21 & 8.11 \\
$\mathrm{Te}$ & 10.71 & 9.26 & 7.73 & 8.59 \\
$\mathrm{~S}$ & 7.95 & 6.63 & 8.31 & 1.40 \\
$\mathrm{Se}$ & 1.16 & 1.49 & 1.54 & 101.82 \\
Total wt. \% & 100.35 & 95.98 & 100.44 & 0.895 \\
\hline $\mathrm{Bi}$ & Empirical formula (calculated on the basis of 2 atoms) & 0.086 \\
$\mathrm{Sb}$ & 0.817 & 0.979 & 0.906 & 0.000 \\
$\mathrm{Fe}$ & 0.090 & 0.039 & 0.086 & 0.016 \\
$\mathrm{Cu}$ & 0.008 & 0.009 & 0.000 & 0.002 \\
$\mathrm{In}$ & 0.019 & 0.013 & 0.015 & 0.102 \\
$\mathrm{~Pb}$ & 0.000 & 0.002 & 0.000 & 1.101 \\
\hline$\Sigma$ & 0.146 & 0.089 & 0.092 & 0.155 \\
\hline $\mathrm{Te}$ & 1.080 & 1.130 & 1.100 & 0.697 \\
$\mathrm{~S}$ & 0.223 & 0.212 & 0.161 & 0.046 \\
$\mathrm{Se}$ & 0.658 & 0.603 & 0.688 & 0.899 \\
\hline$\Sigma$ & 0.039 & 0.055 & 0.052 & 0.900 \\
\hline
\end{tabular}

Table 9. Chemical composition of bismuth (an. 1), bismuthinite (an. 2-8; A-type: an. 2-3, B-type: an. 4-8) and ikunolite (an. 9-13) from Čučma (Majerská valley).

\begin{tabular}{cccccccccccccc}
\hline & $\mathbf{1}$ & $\mathbf{2}$ & $\mathbf{3}$ & $\mathbf{4}$ & $\mathbf{5}$ & $\mathbf{6}$ & $\mathbf{7}$ & $\mathbf{8}$ & $\mathbf{9}$ & $\mathbf{1 0}$ & $\mathbf{1 1}$ & $\mathbf{1 2}$ & $\mathbf{1 3}$ \\
\hline $\mathrm{Bi}$ & 98.40 & 82.77 & 79.70 & 64.51 & 63.16 & 62.54 & 63.08 & 62.96 & 89.77 & 87.93 & 87.48 & 88.69 & 88.72 \\
$\mathrm{Sb}$ & 0.51 & 0.00 & 0.01 & 13.21 & 13.40 & 13.44 & 13.54 & 13.60 & 0.00 & 0.00 & 0.08 & 0.00 & 0.00 \\
$\mathrm{Cu}$ & 0.30 & 0.14 & 0.09 & 0.49 & 0.38 & 0.37 & 0.40 & 0.26 & 0.15 & 0.21 & 0.12 & 0.00 & 0.00 \\
$\mathrm{In}$ & & & & & & & 0.00 & 0.06 & 0.00 & 0.00 & 0.08 & 0.09 & 0.07 \\
$\mathrm{~Pb}$ & 0.66 & 0.66 & 1.55 & 1.66 & 1.26 & 1.22 & 1.29 & 1.05 & 0.00 & 0.04 & 0.04 & 0.00 & 0.00 \\
$\mathrm{~S}$ & & 18.24 & 17.67 & 18.01 & 19.98 & 19.81 & 19.92 & 20.27 & 10.35 & 10.24 & 10.43 & 10.52 & 10.39 \\
$\mathrm{Se}$ & 0.28 & 0.00 & 0.02 & 0.71 & 0.74 & 0.74 & 0.73 & 0.27 & 0.00 & 0.11 & 0.19 & 0.00 & 0.00 \\
$\mathrm{Te}$ & & 0.00 & 0.00 & & & & 0.00 & 0.00 & 0.12 & 0.43 & 0.96 & 0.44 & 0.43 \\
Total wt. \% & 100.15 & 101.81 & 99.04 & 98.59 & 98.92 & 98.12 & 98.97 & 98.47 & 100.27 & 98.53 & 98.42 & 99.30 & 99.18 \\
\hline $\mathrm{Empirical} \mathrm{form}$ ulae & & & & & & & & & & & \\
\hline $\mathrm{Bi}$ & 0.968 & 2.041 & 2.025 & 1.538 & 1.430 & 1.427 & 1.429 & 1.423 & 3.979 & 3.935 & 3.869 & 3.926 & 3.949 \\
$\mathrm{Sb}$ & 0.009 & 0.000 & 0.000 & 0.541 & 0.521 & 0.526 & 0.527 & 0.528 & 0.000 & 0.000 & 0.006 & 0.000 & 0.000 \\
$\mathrm{Cu}$ & 0.010 & 0.011 & 0.007 & 0.039 & 0.029 & 0.028 & 0.030 & 0.019 & 0.022 & 0.031 & 0.017 & 0.000 & 0.000 \\
$\mathrm{In}$ & & & & & & & 0.000 & 0.002 & 0.000 & 0.000 & 0.006 & 0.007 & 0.006 \\
$\mathrm{~Pb}$ & 0.007 & 0.016 & 0.040 & 0.040 & 0.029 & 0.028 & 0.030 & 0.024 & 0.000 & 0.002 & 0.002 & 0.000 & 0.000 \\
\hline $\mathrm{S}$ & 0.993 & 2.069 & 2.072 & 2.157 & 2.008 & 2.009 & 2.015 & 1.997 & 4.001 & 3.968 & 3.901 & 3.933 & 3.954 \\
\hline $\mathrm{S}$ & 0.000 & 2.931 & 2.927 & 2.798 & 2.948 & 2.946 & 2.941 & 2.987 & 2.990 & 2.987 & 3.007 & 3.035 & 3.014 \\
$\mathrm{Se}$ & 0.007 & 0.000 & 0.001 & 0.045 & 0.044 & 0.045 & 0.044 & 0.016 & 0.000 & 0.013 & 0.022 & 0.000 & 0.000 \\
$\mathrm{Te}$ & & 0.000 & 0.000 & 0.000 & 0.000 & 0.000 & 0.000 & 0.000 & 0.009 & 0.032 & 0.070 & 0.032 & 0.031 \\
\hline$\Sigma$ & 0.007 & 2.931 & 2.928 & 2.843 & 2.992 & 2.991 & 2.985 & 3.003 & 2.999 & 3.032 & 3.099 & 3.067 & 3.046 \\
\hline $\mathrm{Bi} / \mathrm{Bi}+\mathrm{Sb})$ & 0.99 & 1.00 & 1.00 & 0.74 & 0.73 & 0.73 & 0.73 & 0.73 & 1.00 & 1.00 & 1.00 & 1.00 & 1.00 \\
\hline
\end{tabular}

Bismuthinite can be divided into two types (A and B), based on the significantly different $\mathrm{Sb}$ contents in both types (Table 9). Small grains (up to $20 \mu \mathrm{m}$ in size) of bis- 
muthinite (type A) grow on needle crystals of minerals of the kobellite-tintinaite series (Figure $7 \mathrm{~d}$ ) or form relicts in a mixture of the supergene minerals, wulfenite and unnamed sulphates of bismuth and lead. The second type of bismuthinite (B) occurs as subhedral grains up to $20 \mu \mathrm{m}$ in size intergrown with minerals of the kobellite-tintinaite series, jamesonite and galena (Figure 7e), or tiny crystals enclosed in kobellite together with native bismuth (Figure 7f). Bismuthinite A is characterized by a practically zero $\mathrm{Sb}$ content and a high content of $\mathrm{Bi}$ (with an average of $81.23 \mathrm{wt}$. \%; $2.03 \mathrm{apfu}$ ), whereas a significant content of $\mathrm{Sb}$ (up to $0.54 \mathrm{apfu}$ ) substituting for Bi is typical for the bismuthinite B. The $\mathrm{Bi} /(\mathrm{Bi}+\mathrm{Sb})$ ratio in the bismuthinite $\mathrm{B}$ is 0.73 . The common admixtures in both types of bismuthinite are $\mathrm{Pb}$ (up to 1.66 wt. \%; $0.04 \mathrm{apfu}$ ) and $\mathrm{Cu}$ (up to 0.49 wt. \%; $0.04 \mathrm{apfu}$ ), and the calculated value of $n_{\text {aik }}$ [31] is between 0.0 and 3.7. Selenium is practically absent in bismuthinite A (with a maximum of $0.02 \mathrm{wt}$. \%), while bismuthinite B contains up to 0.74 wt. \% (0.05 apfu) of Se. The chemical composition of bismuthinite A is expressed by the empirical formula, $\left(\mathrm{Bi}_{2.03} \mathrm{~Pb}_{0.03} \mathrm{Cu}_{0.01}\right)_{\Sigma 2.07} \mathrm{~S}_{2.93}$, and the empirical formula of bismuthinite $\mathrm{B}$ is $\left(\mathrm{Bi}_{1.45} \mathrm{Sb}_{0.53} \mathrm{~Pb}_{0.03} \mathrm{Cu}_{0.03}\right)_{\Sigma 2.04}\left(\mathrm{~S}_{2.92} \mathrm{Se}_{0.04}\right)_{\Sigma 2.96}$.

Bismuth forms anhedral grains up to $10 \mu \mathrm{m}$ in size, enclosed in minerals of the kobellite-tintinaite series together with bismuthinite (Figure 7f). In addition to the dominant $\mathrm{Bi}(98.4 \mathrm{wt}$. \%; $0.97 \mathrm{wt}$ \% \%), only slightly increased contents of $\mathrm{Sb}$ (0.01 apfu), Cu (0.01 apfu), $\mathrm{Pb}(0.01 \mathrm{apfu})$ and Se (0.01 apfu) were found in this mineral phase (Table 9).

Minerals of the kobellite-tintinaite series are relatively abundant at the studied occurrence. They form individual acicular crystals up to $1 \mathrm{~cm}$ long (Figure 9a) or irregular aggregates up to $2 \times 2 \mathrm{~cm}$ enclosed in quartz and are always in close proximity to uraninite and molybdenite veinlets. Kobellite-tintinaite mineral phases were found in close association with pyrite, jamesonite, cosalite, sulphotellurides and ullmannite (Figure $9 \mathrm{~b}$ ) as well as minor amounts of galena and native bismuth. Their aggregates are replaced, to various degrees, by oxyplumboroméite (sometimes complete pseudomorphs) and other unspecified oxides of $\mathrm{Bi}$ and $\mathrm{Pb}$ (Figure $9 \mathrm{~b}, \mathrm{c}$ ). The chemical composition of the studied mineral phases (Table 10) is characterized by a wide interval of $\mathrm{Bi} \leftrightarrow \mathrm{Sb}$ substitution (Figure 10e), ranging compositionally from kobellite (with $\mathrm{Bi}, 12.39$ apfu, $\mathrm{Sb}, 2.95 \mathrm{apfu} ; \mathrm{Bi} /(\mathrm{Bi}+\mathrm{Sb})=0.81$ ) to tintinaite (with $\mathrm{Bi}, 4.63$ apfu; $\mathrm{Sb}, 10.73$ apfu; $\mathrm{Bi} /(\mathrm{Bi}+\mathrm{Sb})=0.29)$. This strong variation is locally causing slightly irregular chemical zoning (Figure $7 \mathrm{~b}$ ). However, most of the studied samples tend to be homogenous and the analyses cluster around the formal boundary between kobellite and tintinaite (Figure 10e).

The calculated value of $N$ (order number of kobellite homologue [32]) is ranging from 1.81 to 2.00 , with an average value of 1.91 . The $\mathrm{Pb}$ content ranges from 33.76 to $37.23 \mathrm{wt}$. \% (9.75-10.92 apfu) and shows a negative correlation with $\mathrm{Cu}$ (up to $1.73 \mathrm{wt}$. \%; $1.62 \mathrm{apfu}$; Figure 10b), but a positive correlation with Fe (up to $2.55 \mathrm{wt}$. \%; $2.55 \mathrm{apfu}$; Figure 10c). The overall $\mathrm{Cu}+\mathrm{Fe}$ content is between 1.90 and $2.68 \mathrm{apfu}$ (2.03 apfu on average), with Fe negatively correlating with $\mathrm{Cu}$ (Figure 10d). Based on the Ag content, the following two groups of minerals of the kobellite-tintinaite series (Figure 10a) can be distinguished: mineral phases with zero Ag content and phases with a slightly increased Ag content (reaching up to 0.24 wt. \%; 0.13 apfu). In the second case, Ag negatively correlates with $\mathrm{Pb}$. A small amount of Se (up to $0.61 \mathrm{wt}$. \%; $0.46 \mathrm{apfu}$ ) is always present in the anionic position and it shows a slightly negative correlation with S (Figure 10f).

Cosalite is an infrequent mineral, and it forms irregular aggregates reaching up to $80 \mu \mathrm{m}$, which are closely associated with minerals of the kobellite-tintinaite series and sulphotellurides (Figure 9d). The studied cosalite has a relatively homogeneous chemical composition (Table 11, Figures 11a and 12). The content of Bi fluctuates in the range of 37.55-38.87 wt. \% (1.71-1.76 apfu). Bismuth is insignificantly substituted by $\mathrm{Sb}$, 3.84-4.52 wt. \% (0.30-0.35 apfu); the $\mathrm{Bi} /(\mathrm{Bi}+\mathrm{Sb})$ ratio ranges from 0.83 to 0.86 . In the anionic position, $\mathrm{S}$ is partially replaced by Se (with a maximum of $1.00 \mathrm{wt} . \%$; $0.12 \mathrm{apfu}$; Figure $11 \mathrm{~b})$. Other admixtures $(\mathrm{Cd}, \mathrm{Cu}, \mathrm{Fe})$ in cosalite are not very important. The content of the above-mentioned elements does not exceed $0.6 \mathrm{wt}$. \% (0.08 apfu) for each element separately. 

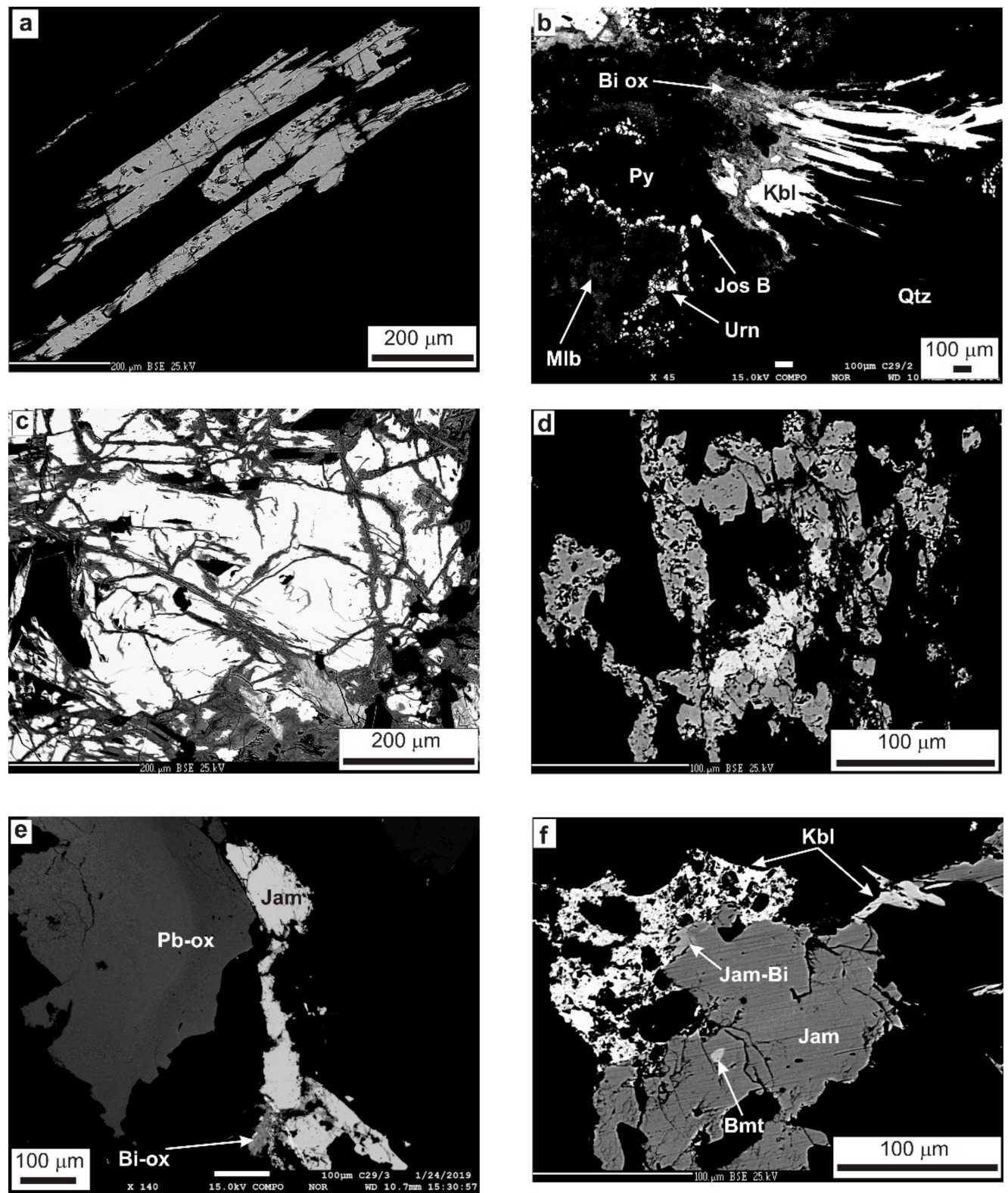

Figure 9. (a) Acicular crystals of kobellite-tintinaite series minerals (grey) in quartz (black). (b) Aggregate of needle-like crystals of kobellite-tintinaite series minerals (Kbl) in association with pyrite (Py), joséite-B (Jos B), ullmannite, uraninite (Urn) and molybdenite (Mlb). Kobellite-tintinaite is partially replaced by supergene oxides of $\mathrm{Bi}-\mathrm{Pb}-\mathrm{Sb}$ ( $\mathrm{Bi}$ ox). The host mineral is quartz (Qtz). (c) Kobellite-tintinaite (white) is replaced by unidentified oxides, $\mathrm{Pb}-\mathrm{Bi}$ (shades of grey). (d) Cosalite (white) associated with minerals of the kobellite-tintinaite series (grey) in quartz (black). (e) Irregular veinlet of jamesonite (Jam) in quartz (black) is replaced by unspecified supergene minerals of $\mathrm{Sb}-\mathrm{Pb}-\mathrm{Bi}$ (Bi-ox). The cavity in the quartz is filled with supergene oxides of $\mathrm{Pb}-\mathrm{Fe}$ (Pb-ox). (f) Jamesonite (Jam, Jam-Bi) in contact with kobellite-tintinaite series minerals with enclosed grain of bismuthinite (Bmt). BSE, photos by T. Mikuš (b,e) and M. Števko (a,c,d,f). 
Table 10. Representative WDS microanalyses of minerals of the kobellite-tintinaite series from Čučma (Majerská valley).

\begin{tabular}{|c|c|c|c|c|c|c|c|c|c|c|c|c|c|c|c|}
\hline & 1 & 2 & 3 & 4 & 5 & 6 & 7 & 8 & 9 & 10 & 11 & 12 & 13 & 14 & 15 \\
\hline $\mathrm{Ag}$ & 0.00 & 0.00 & 0.00 & 0.18 & 0.24 & 0.00 & 0.11 & & 0.02 & 0.00 & & 0.00 & 0.00 & 0.00 & 0.00 \\
\hline $\mathrm{Cu}$ & 0.00 & 0.00 & 0.00 & 1.65 & 1.70 & 1.30 & 1.47 & 1.61 & 1.51 & 1.73 & 1.66 & 1.29 & 0.98 & 0.90 & 0.89 \\
\hline $\mathrm{Fe}$ & 2.29 & 2.29 & 2.55 & 0.44 & 0.42 & 0.79 & 0.57 & 0.38 & 0.66 & 0.46 & 0.35 & 0.68 & 0.86 & 0.96 & 0.89 \\
\hline $\mathrm{Cd}$ & 0.00 & 0.00 & 0.00 & 0.31 & 0.23 & & 0.20 & & & 0.09 & & 0.00 & 0.00 & 0.00 & 0.00 \\
\hline $\mathrm{Pb}$ & 36.70 & 36.09 & 36.26 & 34.28 & 33.38 & 37.23 & 34.70 & 34.56 & 36.68 & 34.12 & 33.76 & 35.74 & 35.20 & 34.66 & 34.75 \\
\hline $\mathrm{Bi}$ & 17.01 & 17.16 & 17.91 & 26.57 & 27.03 & 26.24 & 26.98 & 28.39 & 27.64 & 29.15 & 29.74 & 28.28 & 38.98 & 40.37 & 40.63 \\
\hline $\mathrm{Sb}$ & 23.86 & 23.18 & 22.97 & 17.58 & 17.00 & 15.42 & 15.69 & 15.96 & 15.30 & 15.43 & 15.62 & 14.62 & 7.31 & 5.61 & 5.63 \\
\hline$S$ & 20.38 & 20.12 & 20.33 & 19.12 & 18.97 & 18.25 & 18.38 & 18.81 & 18.46 & 18.89 & 18.58 & 18.61 & 18.19 & 17.58 & 17.54 \\
\hline Se & 0.15 & 0.19 & 0.11 & 0.23 & 0.16 & 0.10 & 0.20 & 0.50 & 0.41 & 0.18 & 0.61 & 0.23 & 0.23 & 0.28 & 0.27 \\
\hline Total wt. \% & 100.39 & 99.03 & 100.13 & 100.36 & 99.13 & 99.32 & 98.30 & 100.21 & 100.68 & 100.05 & 100.32 & 99.45 & 101.75 & 100.36 & 100.60 \\
\hline \multicolumn{16}{|c|}{ Empirical formula (calculated on the basis of 63 atoms) } \\
\hline $\mathrm{Ag}$ & 0.000 & 0.000 & 0.000 & 0.098 & 0.132 & 0.000 & 0.062 & 0.000 & 0.010 & 0.000 & 0.000 & 0.000 & 0.000 & 0.000 & 0.000 \\
\hline $\mathrm{Cu}$ & 0.000 & 0.000 & 0.000 & 1.522 & 1.586 & 1.239 & 1.405 & 1.507 & 1.423 & 1.620 & 1.563 & 1.225 & 0.955 & 0.901 & 0.892 \\
\hline $\mathrm{Fe}$ & 2.280 & 2.311 & 2.545 & 0.462 & 0.446 & 0.855 & 0.620 & 0.405 & 0.706 & 0.490 & 0.375 & 0.735 & 0.953 & 1.094 & 1.015 \\
\hline $\mathrm{Cd}$ & 0.000 & 0.000 & 0.000 & 0.162 & 0.121 & 0.000 & 0.108 & 0.000 & 0.000 & 0.048 & 0.000 & 0.000 & 0.000 & 0.000 & 0.000 \\
\hline$\Sigma$ & 2.280 & 2.311 & 2.545 & 2.244 & 2.286 & 2.094 & 2.195 & 1.912 & 2.139 & 2.158 & 1.938 & 1.960 & 1.908 & 1.996 & 1.908 \\
\hline $\mathrm{Pb}$ & 9.848 & 9.818 & 9.752 & 9.700 & 9.554 & 10.917 & 10.170 & 9.924 & 10.602 & 9.800 & 9.747 & 10.411 & 10.518 & 10.647 & 10.685 \\
\hline $\mathrm{Bi}$ & 4.526 & 4.629 & 4.776 & 7.454 & 7.670 & 7.629 & 7.840 & 8.082 & 7.921 & 8.301 & 8.513 & 8.168 & 11.548 & 12.296 & 12.387 \\
\hline $\mathrm{Sb}$ & 10.896 & 10.731 & 10.513 & 8.465 & 8.280 & 7.693 & 7.825 & 7.798 & 7.528 & 7.541 & 7.674 & 7.247 & 3.717 & 2.933 & 2.946 \\
\hline$\Sigma$ & 15.421 & 15.360 & 15.289 & 15.919 & 15.950 & 15.322 & 15.666 & 15.881 & 15.449 & 15.842 & 16.187 & 15.416 & 15.265 & 15.229 & 15.333 \\
\hline$S$ & 35.345 & 35.375 & 35.337 & 34.966 & 35.090 & 34.589 & 34.816 & 34.907 & 34.496 & 35.064 & 34.667 & 35.037 & 35.128 & 34.903 & 34.857 \\
\hline Se & 0.106 & 0.136 & 0.078 & 0.171 & 0.120 & 0.077 & 0.154 & 0.377 & 0.314 & 0.136 & 0.462 & 0.176 & 0.180 & 0.226 & 0.218 \\
\hline$\Sigma$ & 35.450 & 35.511 & 35.415 & 35.137 & 35.210 & 34.666 & 34.969 & 35.283 & 34.810 & 35.200 & 35.129 & 35.213 & 35.308 & 35.128 & 35.074 \\
\hline $\mathrm{Bi} /(\mathrm{Sb}+\mathrm{Bi})$ & 0.29 & 0.30 & 0.31 & 0.47 & 0.48 & 0.50 & 0.50 & 0.51 & 0.51 & 0.52 & 0.53 & 0.53 & 0.76 & 0.81 & 0.81 \\
\hline $\mathrm{Sb} /(\mathrm{Sb}+\mathrm{Bi})$ & 0.71 & 0.70 & 0.69 & 0.53 & 0.52 & 0.50 & 0.50 & 0.49 & 0.49 & 0.48 & 0.47 & 0.47 & 0.24 & 0.19 & 0.19 \\
\hline
\end{tabular}
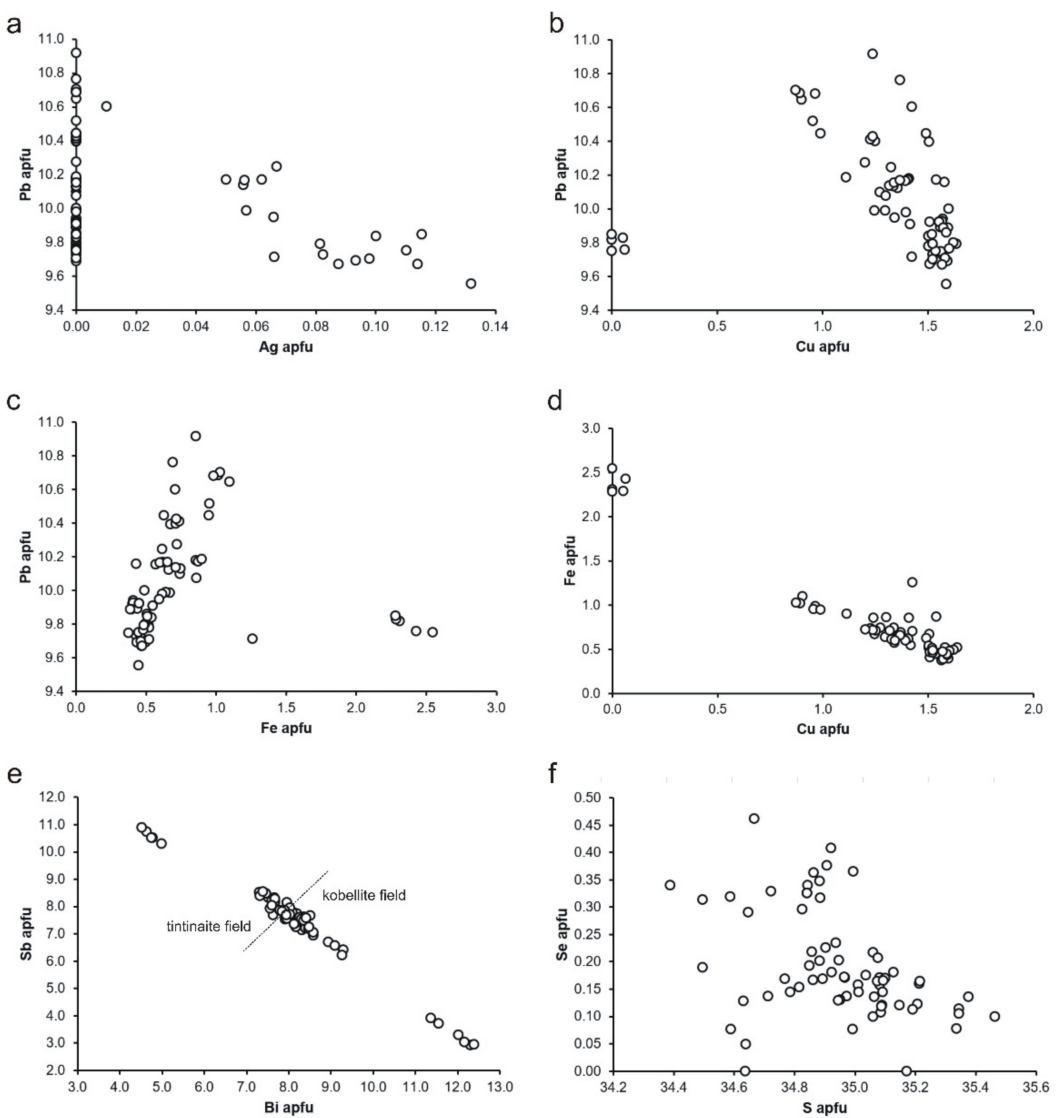

Figure 10. Interdependencies of elements in minerals of kobellite-tintinaite series: (a) Ag vs. $\mathrm{Pb}$; (b) $\mathrm{Cu}$ vs. $\mathrm{Pb}$; (c) Fe vs. Pb; (d) Cu vs. Fe; (e) Bi vs. Sb; (f) S vs. Se. 
Table 11. Representative WDS microanalyses of cosalite from Čučma (Majerská valley).

\begin{tabular}{|c|c|c|c|c|c|c|c|c|c|c|}
\hline & 1 & 2 & 3 & 4 & 5 & 6 & 7 & 8 & 9 & 10 \\
\hline $\mathrm{Pb}$ & 39.91 & 39.85 & 40.50 & 40.13 & 40.04 & 39.75 & 39.75 & 40.29 & 40.06 & 40.20 \\
\hline Mn & 0.08 & 0.07 & 0.08 & 0.09 & 0.08 & 0.10 & 0.08 & 0.10 & 0.10 & 0.07 \\
\hline $\mathrm{Fe}$ & 0.15 & 0.13 & 0.16 & 0.14 & 0.15 & 0.13 & 0.11 & 0.15 & 0.14 & 0.14 \\
\hline $\mathrm{Cu}$ & 0.13 & 0.15 & 0.18 & 0.16 & 0.16 & 0.51 & 0.43 & 0.16 & 0.18 & 0.16 \\
\hline $\mathrm{Cd}$ & 0.44 & 0.44 & 0.48 & 0.50 & 0.47 & 0.42 & 0.39 & 0.50 & 0.49 & 0.49 \\
\hline In & 0.06 & 0.06 & 0.06 & 0.08 & 0.00 & 0.07 & 0.00 & 0.07 & 0.00 & 0.07 \\
\hline $\mathrm{Sb}$ & 4.09 & 4.07 & 4.45 & 4.52 & 4.15 & 3.84 & 3.86 & 4.15 & 4.26 & 4.38 \\
\hline $\mathrm{Bi}$ & 37.89 & 38.08 & 38.62 & 38.01 & 37.88 & 38.87 & 38.65 & 37.55 & 38.16 & 38.39 \\
\hline S & 16.87 & 16.73 & 16.88 & 16.83 & 16.38 & 16.42 & 16.49 & 16.65 & 16.64 & 16.96 \\
\hline Se & 0.33 & 0.41 & 0.41 & 0.42 & 0.41 & 1.00 & 0.78 & 0.37 & 0.41 & 0.41 \\
\hline Total wt. \% & 99.95 & 99.99 & 101.82 & 100.88 & 99.72 & 101.11 & 100.54 & 99.99 & 100.44 & 101.27 \\
\hline \multicolumn{11}{|c|}{ Empirical formula (calculated on the basis of 9 atoms) } \\
\hline $\mathrm{Pb}$ & 1.828 & 1.831 & 1.832 & 1.826 & 1.859 & 1.816 & 1.824 & 1.853 & 1.838 & 1.820 \\
\hline Mn & 0.014 & 0.012 & 0.014 & 0.015 & 0.014 & 0.017 & 0.014 & 0.017 & 0.017 & 0.012 \\
\hline $\mathrm{Fe}$ & 0.025 & 0.022 & 0.027 & 0.024 & 0.026 & 0.022 & 0.019 & 0.026 & 0.024 & 0.024 \\
\hline $\mathrm{Cu}$ & 0.019 & 0.022 & 0.027 & 0.024 & 0.024 & 0.076 & 0.064 & 0.024 & 0.027 & 0.024 \\
\hline $\mathrm{Cd}$ & 0.037 & 0.037 & 0.040 & 0.042 & 0.040 & 0.035 & 0.033 & 0.042 & 0.041 & 0.041 \\
\hline In & 0.005 & 0.005 & 0.005 & 0.007 & 0.000 & 0.006 & 0.000 & 0.006 & 0.000 & 0.006 \\
\hline$\Sigma$ & 1.928 & 1.930 & 1.944 & 1.937 & 1.963 & 1.972 & 1.954 & 1.968 & 1.948 & 1.926 \\
\hline $\mathrm{Sb}$ & 0.319 & 0.318 & 0.342 & 0.350 & 0.328 & 0.299 & 0.301 & 0.325 & 0.333 & 0.338 \\
\hline $\mathrm{Bi}$ & 1.720 & 1.735 & 1.732 & 1.715 & 1.744 & 1.761 & 1.759 & 1.712 & 1.736 & 1.724 \\
\hline$\Sigma$ & 2.039 & 2.053 & 2.074 & 2.064 & 2.072 & 2.059 & 2.060 & 2.037 & 2.069 & 2.061 \\
\hline$S$ & 4.993 & 4.968 & 4.934 & 4.948 & 4.915 & 4.848 & 4.891 & 4.950 & 4.934 & 4.964 \\
\hline Se & 0.040 & 0.049 & 0.049 & 0.050 & 0.050 & 0.120 & 0.094 & 0.045 & 0.049 & 0.049 \\
\hline$\Sigma$ & 5.032 & 5.017 & 4.982 & 4.999 & 4.965 & 4.968 & 4.985 & 4.994 & 4.984 & 5.013 \\
\hline $\mathrm{Bi} /(\mathrm{Bi}+\mathrm{Sb})$ & 0.84 & 0.84 & 0.83 & 0.83 & 0.84 & 0.86 & 0.85 & 0.84 & 0.84 & 0.84 \\
\hline
\end{tabular}

a

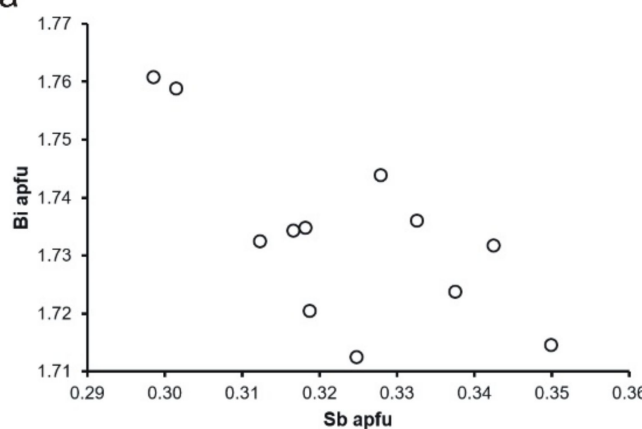

b

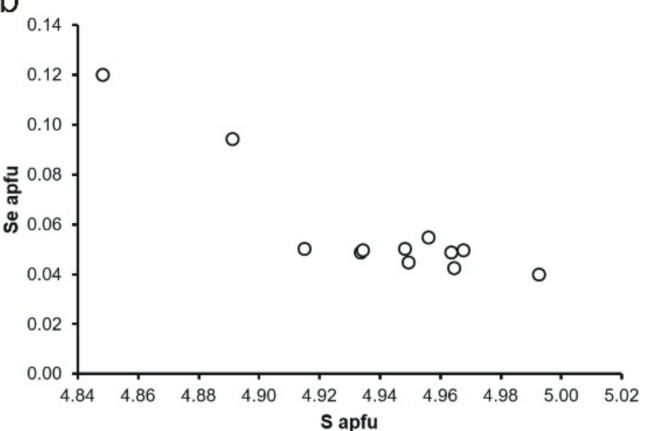

Figure 11. Interdependence of Sb vs. Bi (a) and S vs. Se (b) in cosalite from Čučma (Majerská valley).

Jamesonite forms irregular veinlets (with a length of up to $1 \mathrm{~mm}$ ) in quartz (Figure 9e) or subhedral grains and aggregates up to $150 \mu \mathrm{m}$ that are intimately intergrown with minerals of the kobellite-tintinaite series (Figure 9f). Supergene Fe-Pb-Bi oxides fill the fissures in the jamesonite or replace it. In terms of chemical composition, the studied jamesonite is interesting mainly due to the relatively stable admixture of $\mathrm{Bi}$ (with an average of 16.48 wt. \%; 1.77 apfu; Table 12). Rarely, the Bi content reaches up to 24.23 wt. \% (2.67 apfu) and it causes slight chemical zoning (Figure 9f). The Sb content averages around $23.45 \mathrm{wt}$. \% (4.32 apfu), and in Bi-rich jamesonite the Sb content decreases to 17.87 wt. \% (3.38 apfu). Weakly increased contents of $\mathrm{Cu}$ (up to 0.15 wt. \%; 0.05 apfu), As (up to 0.13 wt. \%; 0.04 apfu) and Se (with a maximum of 0.40 wt. \%; 0.12 apfu) were also 
detected. The chemical composition of the studied jamesonite corresponds to the following empirical formulae:

$\left(\mathrm{Fe}_{0.96} \mathrm{Mn}_{0.01} \mathrm{Cu}_{0.01}\right)_{\Sigma 0.98} \mathrm{~Pb}_{3.92}\left(\mathrm{Sb}_{4.32} \mathrm{Bi}_{1.77}\right)_{\Sigma 6.09}\left(\mathrm{~S}_{13.97} \mathrm{Se}_{0.03}\right)_{\Sigma 14.00}$, and

$\left(\mathrm{Fe}_{0.92} \mathrm{Cu}_{0.05}\right)_{\Sigma 0.97} \mathrm{~Pb}_{3.86}\left(\mathrm{Sb}_{3.38} \mathrm{Bi}_{2.67}\right)_{\Sigma 6.06}\left(\mathrm{~S}_{13.99} \mathrm{Se}_{0.12}\right)_{\Sigma 14.11}$ (so-called "sakharovaite", Figure 12).

Table 12. Chemical composition of jamesonite from Čučma (Majerská valley).

\begin{tabular}{|c|c|c|c|c|c|c|c|c|c|c|c|}
\hline & 1 & 2 & 3 & 4 & 5 & 6 & 7 & 8 & 9 & 10 & 11 \\
\hline $\mathrm{Fe}$ & 2.38 & 2.40 & 2.36 & 2.37 & 2.39 & 2.62 & 2.46 & 2.29 & 2.32 & 2.23 & 0.48 \\
\hline $\mathrm{Mn}$ & & & 0.05 & 0.00 & 0.07 & 0.05 & 0.06 & 0.05 & 0.05 & 0.00 & 0 \\
\hline $\mathrm{Cu}$ & & & 0.00 & 0.00 & 0.00 & 0.08 & 0.07 & 0.00 & 0.00 & 0.15 & 1.55 \\
\hline $\mathrm{Pb}$ & 36.70 & 36.70 & 36.14 & 35.69 & 36.12 & 36.33 & 36.56 & 36.43 & 35.70 & 34.72 & 33.47 \\
\hline As & 0.02 & 0.13 & & & & & & & & & \\
\hline $\mathrm{Sb}$ & 23.48 & 23.33 & 23.15 & 23.53 & 23.58 & 23.56 & 23.68 & 23.67 & 23.08 & 17.87 & 16.14 \\
\hline $\mathrm{Bi}$ & 16.15 & 16.28 & 16.68 & 16.37 & 16.70 & 16.61 & 16.37 & 16.44 & 16.69 & 24.23 & 27.59 \\
\hline$S$ & 19.52 & 19.39 & 20.11 & 20.21 & 20.42 & 20.27 & 20.05 & 19.93 & 20.10 & 19.45 & 18.81 \\
\hline Se & & & 0.17 & 0.15 & 0.15 & 0.14 & 0.19 & 0.17 & 0.14 & 0.40 & 0.24 \\
\hline Total wt. \% & 98.25 & 98.22 & 98.66 & 98.32 & 99.43 & 99.66 & 99.44 & 98.98 & 98.08 & 99.05 & 98.28 \\
\hline \multicolumn{12}{|c|}{ Empirical formula (calculated on the basis of 25 atoms) } \\
\hline $\mathrm{Fe}$ & 0.970 & 0.979 & 0.946 & 0.949 & 0.946 & 1.037 & 0.980 & 0.920 & 0.933 & 0.921 & 0.205 \\
\hline $\mathrm{Mn}$ & & & 0.020 & 0.000 & 0.028 & 0.020 & 0.024 & 0.020 & 0.020 & 0.000 & 0.000 \\
\hline $\mathrm{Cu}$ & & & 0.000 & 0.000 & 0.000 & 0.028 & 0.025 & 0.000 & 0.000 & 0.054 & 0.581 \\
\hline$\Sigma$ & 0.970 & 0.979 & 0.966 & 0.949 & 0.974 & 1.085 & 1.029 & 0.940 & 0.954 & 0.975 & 0.786 \\
\hline $\mathrm{Pb}$ & 4.029 & 4.040 & 3.904 & 3.850 & 3.854 & 3.874 & 3.927 & 3.944 & 3.870 & 3.863 & 3.850 \\
\hline As & 0.006 & 0.038 & & & & & & & & & \\
\hline $\mathrm{Sb}$ & 4.387 & 4.371 & 4.256 & 4.319 & 4.281 & 4.275 & 4.328 & 4.360 & 4.258 & 3.384 & 3.160 \\
\hline $\mathrm{Bi}$ & 1.758 & 1.777 & 1.786 & 1.751 & 1.767 & 1.756 & 1.743 & 1.764 & 1.794 & 2.673 & 3.147 \\
\hline$\Sigma$ & 6.152 & 6.186 & 6.042 & 6.070 & 6.048 & 6.032 & 6.072 & 6.125 & 6.052 & 6.057 & 6.306 \\
\hline$S$ & 13.848 & 13.796 & 14.040 & 14.089 & 14.081 & 13.970 & 13.919 & 13.943 & 14.084 & 13.988 & 13.985 \\
\hline Se & & & 0.048 & 0.042 & 0.042 & 0.039 & 0.054 & 0.048 & 0.040 & 0.117 & 0.072 \\
\hline$\Sigma$ & 13.848 & 13.796 & 14.088 & 14.132 & 14.123 & 14.009 & 13.972 & 13.992 & 14.124 & 14.104 & 14.057 \\
\hline $\mathrm{Sb} /(\mathrm{Sb}+\mathrm{Bi})$ & 0.71 & 0.71 & 0.70 & 0.71 & 0.71 & 0.71 & 0.71 & 0.71 & 0.70 & 0.56 & 0.50 \\
\hline
\end{tabular}

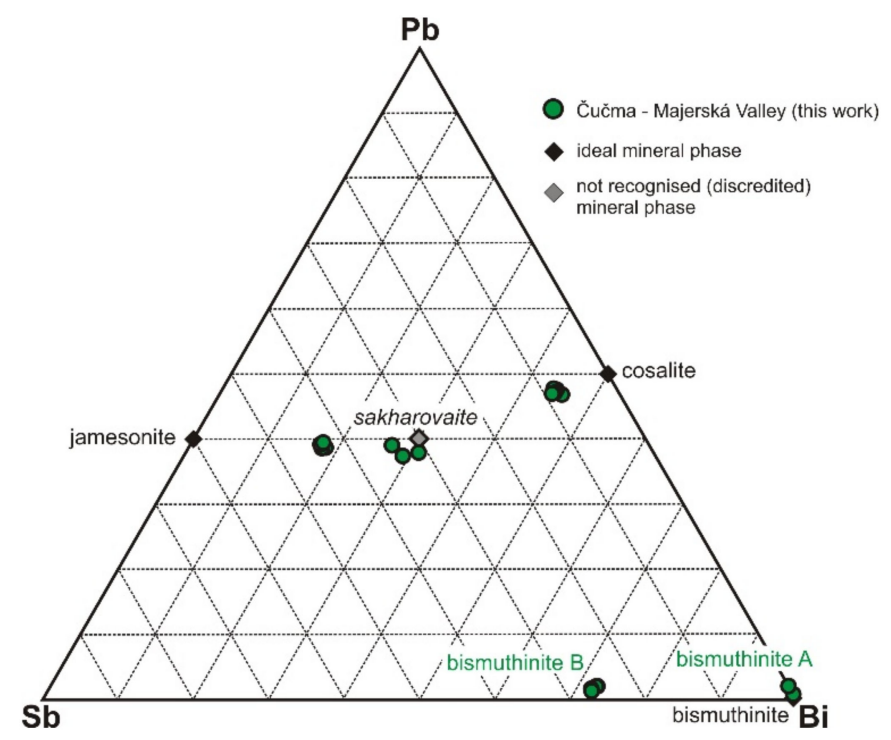

Figure 12. Chemical composition of bismuthinite, Sb-bearing cosalite and Bi-bearing jamesonite from Čučma, in ternary system $\mathrm{Sb}-\mathrm{Bi}-\mathrm{Pb}$. 
Galena occurs as subhedral grains up to $20 \mu \mathrm{m}$ in size or aggregates intimately intergrown with bismuthinite and minerals of the kobellite-tintinaite series (Figure 7e). The chemical composition of galena is given in Table 13. From the admixtures, Bi (up to 0.01 apfu) and Se (up to $0.07 \mathrm{apfu}$ ) are relatively important, while the content of other elements is insignificant. The average empirical formula of the studied galena is $\left(\mathrm{Pb}_{0.97} \mathrm{Bi}_{0.01}\right)_{\Sigma 0.98}\left(\mathrm{~S}_{0.96} \mathrm{Se}_{0.07}\right)_{\Sigma 1.03}$.

Table 13. Chemical composition of galena from Čučma (Majerská valley).

\begin{tabular}{ccc}
\hline & $\mathbf{1}$ & $\mathbf{2}$ \\
\hline $\mathrm{Pb}$ & 83.76 & 83.41 \\
$\mathrm{Fe}$ & 0.00 & 0.05 \\
$\mathrm{Cu}$ & 0.09 & 0.06 \\
$\mathrm{In}$ & 0.06 & 0.06 \\
$\mathrm{Bi}$ & 0.70 & 0.57 \\
$\mathrm{~S}$ & 12.81 & 12.79 \\
$\mathrm{Se}$ & 2.11 & 2.32 \\
Total wt. \% & 99.55 & 99.27 \\
\hline $\mathrm{Fb}$ & Empirical formula (on 2 atoms) & 0.963 \\
$\mathrm{Fe}$ & 0.967 & 0.002 \\
$\mathrm{Cu}$ & 0.000 & 0.002 \\
$\mathrm{In}$ & 0.004 & 0.001 \\
$\mathrm{Bi}$ & 0.001 & 0.007 \\
\hline$\Sigma$ & 0.008 & 0.975 \\
\hline $\mathrm{S}$ & 0.980 & 0.954 \\
$\mathrm{Se}$ & 0.956 & 0.070 \\
\hline $\mathrm{S}$ & 0.064 & 1.025 \\
\hline
\end{tabular}

\subsubsection{Non-Ore and Accessory Minerals}

Quartz is a dominant component of the gangue. In the transmitted light, the two generations of quartz were identified. Quartz I forms weakly undulous, anhedral grains (up to $5 \mathrm{~mm}$ in size), embedded in quartz II. Edges of these grains are partially melted and recrystallized. Quartz II is an essential part of the gangue. It is fine-grained, the size of individual anhedral grains is around $0.1-0.2 \mathrm{~mm}$. The undulosity was not observed. Ore minerals are accumulated mainly at the interfaces of quartz I and II aggregates but penetrate both quartz generations.

Fluorapatite is a typical and the most wide-spread accessory mineral of the gangue. It forms subhedral crystals (with square cross-sections), or anhedral grains up to $0.5 \mathrm{~mm}$ in size, irregularly scattered in both generations of quartz or in close spatial association with U-Mo minerals (Figures $4 \mathrm{~d}$ and 13a). Therefore, this position probably indicates at least two generations of fluorapatite. Locally, fluorapatite is overgrown with aggregates of uraninite, and cracks or cavities in fluorapatite are filled with supergene uranyl minerals (Figure 13b). Fluorapatite from the Majerská valley is generally characterized by an overall low content of admixtures and, besides $\mathrm{Ca}$ and $\mathrm{P}$ (Table 14), only minor amounts of $\mathrm{Y}$ and REE are present. The average content of $\mathrm{Y}_{2} \mathrm{O}_{3}+\mathrm{REE}_{2} \mathrm{O}_{3}$ in fluorapatite is 0.51 wt. \% (0.02 apfu; Y + REE). Except of Y, LREE (0.28 wt. \% LREE $\mathrm{O}_{3} ; 0.01$ apfu LREE) slightly predominates over HREE (0.15 wt. \% $\mathrm{HREE}_{2} \mathrm{O}_{3} ; 0.004$ apfu HREE). The hydroxyl groups $(\mathrm{OH})^{-}$are locally indistinctly present (reaching up to $0.08 \mathrm{apfu}$ ), at the expense of the reduced $\mathrm{F}$ content. The average empirical formula of studied fluorapatite is $\left(\mathrm{Ca}_{4.92} \mathrm{Y}+\right.$ $\left.\mathrm{REE}_{0.02}\right)_{\Sigma 4.94}\left(\mathrm{PO}_{4}\right)_{3.01}\left(\mathrm{~F}_{1.01} \mathrm{OH}_{0.03}\right)_{\Sigma 1.04}$. 

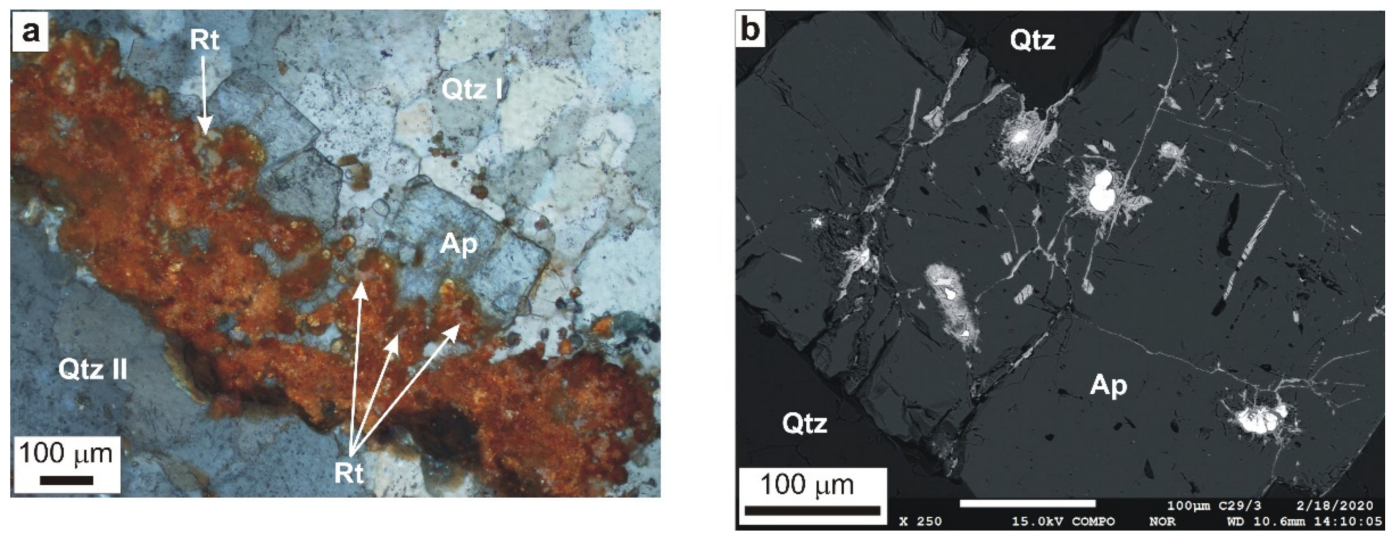

Figure 13. (a) Subhedral crystals of fluorapatite (Ap) in quartz (Qtz), replaced by unspecified supergene U mineral (orangebrown), which represent complete pseudomorphosis after the original uraninite veinlet. Small grains of rutile (Rt) are also enclosed by supergene U mineral. (b) Small cavities and cracks in fluorapatite (Ap) are filled with uraninite (white) and supergene uranyl minerals (light grey). The host mineral is quartz (Qtz). Picture a-transmitted light, PPL (photo: S. Ferenc), picture b-BSE (photo: T. Mikuš).

Zircon was found mainly in quartz II. It forms thin, long prismatic crystals with lengths of up to $50 \mu \mathrm{m}$. Aggregates of flaky crystals of unspecified chlorites fill thin fissures or small cavities in the quartz. Chlorite aggregates are also developed in interstitial spaces between ore minerals (uraninite, molybdenite, tetradymite; Figure 7a). Small veinlets of titanite rarely cut molybdenite veins, enclosing aggregates of molybdenite crystals (Figure 7a). Rutile forms irregular grains (up to $50 \mu \mathrm{m}$ in size), enclosed in uraninite and molybdenite, respectively, by supergene uranyl minerals (Figure 13a). Minerals of the tourmaline group (schorl to dravite) occur as individual acicular crystals up to $3 \mathrm{~mm}$ or as irregular nests up to $1.5 \mathrm{~cm}$ in size enclosed in quartz.

Table 14. Representative WDS microanalyses of fluorapatite from Čučma (Majerská valley).

\begin{tabular}{|c|c|c|c|c|c|c|}
\hline & 1 & 2 & 3 & 4 & 5 & 6 \\
\hline $\mathrm{Na}_{2} \mathrm{O}$ & 0.04 & 0.03 & 0.03 & 0.04 & 0.01 & 0.03 \\
\hline $\mathrm{CaO}$ & 55.03 & 55.15 & 55.17 & 55.47 & 55.33 & 55.49 \\
\hline $\mathrm{BaO}$ & 0.29 & 0.00 & 0.00 & 0.12 & 0.00 & 0.00 \\
\hline $\mathrm{MnO}$ & 0.01 & 0.00 & 0.04 & 0.00 & 0.00 & 0.04 \\
\hline $\mathrm{FeO}$ & 0.00 & 0.00 & 0.02 & 0.10 & 0.04 & 0.01 \\
\hline $\mathrm{PbO}$ & 0.00 & 0.00 & 0.00 & 0.01 & 0.00 & 0.02 \\
\hline $\mathrm{Al}_{2} \mathrm{O}_{3}$ & 0.02 & 0.00 & 0.00 & 0.01 & 0.00 & 0.00 \\
\hline $\mathrm{Y}_{2} \mathrm{O}_{3}$ & 0.08 & 0.08 & 0.08 & 0.01 & 0.13 & 0.09 \\
\hline $\mathrm{La}_{2} \mathrm{O}_{3}$ & 0.05 & 0.00 & 0.03 & 0.01 & 0.06 & 0.00 \\
\hline $\mathrm{Ce}_{2} \mathrm{O}_{3}$ & 0.04 & 0.06 & 0.08 & 0.07 & 0.09 & 0.08 \\
\hline $\mathrm{Pr}_{2} \mathrm{O}_{3}$ & 0.01 & 0.01 & 0.10 & 0.00 & 0.00 & 0.06 \\
\hline $\mathrm{Nd}_{2} \mathrm{O}_{3}$ & 0.03 & 0.05 & 0.06 & 0.06 & 0.05 & 0.12 \\
\hline $\mathrm{Sm}_{2} \mathrm{O}_{3}$ & 0.02 & 0.00 & 0.00 & 0.07 & 0.12 & 0.03 \\
\hline $\mathrm{Eu}_{2} \mathrm{O}_{3}$ & 0.00 & 0.02 & 0.04 & 0.00 & 0.01 & 0.04 \\
\hline $\mathrm{Gd}_{2} \mathrm{O}_{3}$ & 0.10 & 0.08 & 0.04 & 0.04 & 0.01 & 0.00 \\
\hline $\mathrm{Tb}_{2} \mathrm{O}_{3}$ & 0.01 & 0.02 & 0.00 & 0.02 & 0.00 & 0.06 \\
\hline $\mathrm{Dy}_{2} \mathrm{O}_{3}$ & 0.08 & 0.00 & 0.00 & 0.00 & 0.12 & 0.03 \\
\hline $\mathrm{Ho}_{2} \mathrm{O}_{3}$ & 0.10 & 0.04 & 0.01 & 0.04 & 0.12 & 0.00 \\
\hline $\mathrm{Er}_{2} \mathrm{O}_{3}$ & 0.02 & 0.02 & 0.00 & 0.00 & 0.08 & 0.00 \\
\hline $\mathrm{Lu}_{2} \mathrm{O}_{3}$ & 0.00 & 0.00 & 0.04 & 0.02 & 0.09 & 0.00 \\
\hline $\mathrm{TiO}_{2}$ & 0.06 & 0.00 & 0.09 & 0.01 & 0.09 & 0.11 \\
\hline $\mathrm{ZrO}_{2}$ & 0.06 & 0.11 & 0.05 & 0.06 & 0.10 & 0.11 \\
\hline $\mathrm{ThO}_{2}$ & 0.01 & 0.00 & 0.00 & 0.01 & 0.00 & 0.00 \\
\hline $\mathrm{UO}_{2}$ & 0.00 & 0.02 & 0.02 & 0.00 & 0.00 & 0.01 \\
\hline $\mathrm{SiO}_{2}$ & 0.04 & 0.03 & 0.00 & 0.01 & 0.00 & 0.01 \\
\hline $\mathrm{P}_{2} \mathrm{O}_{5}$ & 42.82 & 43.00 & 42.42 & 43.06 & 42.63 & 42.88 \\
\hline
\end{tabular}


Table 14. Cont.

\begin{tabular}{|c|c|c|c|c|c|c|}
\hline & 1 & 2 & 3 & 4 & 5 & 6 \\
\hline $\mathrm{As}_{2} \mathrm{O}_{5}$ & 0.01 & 0.10 & 0.02 & 0.00 & 0.03 & 0.00 \\
\hline $\mathrm{SO}_{3}$ & 0.01 & 0.02 & 0.00 & 0.03 & 0.02 & 0.01 \\
\hline $\mathrm{H}_{2} \mathrm{O}$ * & & & & 0.31 & 0.27 & 0.04 \\
\hline F & 4.21 & 4.20 & 3.89 & 3.47 & 3.50 & 3.77 \\
\hline Total wt. \% & 103.15 & 103.01 & 102.23 & 103.03 & 102.86 & 103.03 \\
\hline $\mathrm{F}=\mathrm{O}$ & -1.77 & -1.77 & -1.64 & -1.46 & -1.47 & -1.59 \\
\hline Total wt. $\%$ & 101.37 & 101.25 & 100.59 & 101.57 & 101.39 & 101.45 \\
\hline \multicolumn{7}{|c|}{ Empirical formula (calculated on the basis of 13 anions) } \\
\hline $\mathrm{Na}$ & 0.006 & 0.005 & 0.004 & 0.006 & 0.002 & 0.004 \\
\hline $\mathrm{Ca}$ & 4.882 & 4.883 & 4.937 & 4.934 & 4.945 & 4.928 \\
\hline $\mathrm{Ba}$ & 0.009 & 0.000 & 0.000 & 0.004 & 0.000 & 0.000 \\
\hline $\mathrm{Mn}$ & 0.001 & 0.000 & 0.003 & 0.000 & 0.000 & 0.003 \\
\hline $\mathrm{Fe}$ & 0.000 & 0.000 & 0.002 & 0.007 & 0.003 & 0.000 \\
\hline $\mathrm{Pb}$ & 0.000 & 0.000 & 0.000 & 0.000 & 0.000 & 0.000 \\
\hline $\mathrm{Al}$ & 0.002 & 0.000 & 0.000 & 0.001 & 0.000 & 0.000 \\
\hline $\mathrm{Y}$ & 0.004 & 0.004 & 0.004 & 0.000 & 0.006 & 0.004 \\
\hline $\mathrm{La}$ & 0.002 & 0.000 & 0.001 & 0.000 & 0.002 & 0.000 \\
\hline $\mathrm{Ce}$ & 0.001 & 0.002 & 0.002 & 0.002 & 0.003 & 0.002 \\
\hline $\operatorname{Pr}$ & 0.000 & 0.000 & 0.003 & 0.000 & 0.000 & 0.002 \\
\hline $\mathrm{Nd}$ & 0.001 & 0.001 & 0.002 & 0.002 & 0.001 & 0.004 \\
\hline $\mathrm{Sm}$ & 0.001 & 0.000 & 0.000 & 0.002 & 0.003 & 0.001 \\
\hline $\mathrm{Eu}$ & 0.000 & 0.001 & 0.001 & 0.000 & 0.000 & 0.001 \\
\hline Gd & 0.003 & 0.002 & 0.001 & 0.001 & 0.000 & 0.000 \\
\hline $\mathrm{Tb}$ & 0.000 & 0.001 & 0.000 & 0.000 & 0.000 & 0.002 \\
\hline Dy & 0.002 & 0.000 & 0.000 & 0.000 & 0.003 & 0.001 \\
\hline Ho & 0.003 & 0.001 & 0.000 & 0.001 & 0.003 & 0.000 \\
\hline Er & 0.001 & 0.000 & 0.000 & 0.000 & 0.002 & 0.000 \\
\hline $\mathrm{Lu}$ & 0.000 & 0.000 & 0.001 & 0.001 & 0.002 & 0.000 \\
\hline $\mathrm{Ti}$ & 0.004 & 0.000 & 0.006 & 0.001 & 0.006 & 0.007 \\
\hline $\mathrm{Zr}$ & 0.003 & 0.005 & 0.002 & 0.002 & 0.004 & 0.004 \\
\hline Th & 0.000 & 0.000 & 0.000 & 0.000 & 0.000 & 0.000 \\
\hline $\mathrm{U}$ & 0.000 & 0.000 & 0.000 & 0.000 & 0.000 & 0.000 \\
\hline$\Sigma \mathrm{M}$ & 4.923 & 4.904 & 4.970 & 4.964 & 4.985 & 4.964 \\
\hline $\mathrm{Si}$ & 0.003 & 0.002 & 0.000 & 0.001 & 0.000 & 0.001 \\
\hline $\mathrm{P}$ & 3.001 & 3.008 & 3.000 & 3.027 & 3.010 & 3.009 \\
\hline As & 0.000 & 0.004 & 0.001 & 0.000 & 0.001 & 0.000 \\
\hline S & 0.001 & 0.001 & 0.000 & 0.002 & 0.001 & 0.001 \\
\hline$\Sigma \mathrm{T}$ & 3.006 & 3.016 & 3.001 & 3.030 & 3.012 & 3.010 \\
\hline $\mathrm{F}$ & 1.102 & 1.098 & 1.028 & 0.911 & 0.923 & 0.988 \\
\hline $\mathrm{OH}$ & & & & 0.089 & 0.077 & 0.012 \\
\hline$\Sigma X$ & 1.102 & 1.098 & 1.028 & 1.000 & 1.000 & 1.000 \\
\hline
\end{tabular}

${ }^{*} \mathrm{H}_{2} \mathrm{O}$ content is calculated based on the number of $\mathrm{OH}$ in empirical formula.

\section{Discussion}

\subsection{Succession of Mineralization}

The microstructural relationships of vein filling minerals suggest that the studied mineralization originated, most probably, in the following stages:

(I.) quartz I, fluorapatite I;

(II.) quartz II, fluorapatite II, zircon, rutile chlorite, tourmaline;

(III. uraninite, molybdenite, U-Ti oxides;

(IV.) (?) pyrite I (non-zonal), ullmannite, gersdorffite, cobaltite;

(Va.) (?) galena, bismuth, tetradymite, joséite $\mathrm{A}$ and $\mathrm{B}, \mathrm{Bi}_{3}(\mathrm{TeS})_{2}$ mineral phase, $(\mathrm{BiPb})(\mathrm{TeS})$ mineral phase, ikunolite;

(Vb.) kobellite-tintinaite series mineral phases, cosalite; 
(VI.) pyrite II (zonal);

(VII.) titanite, chlorite;

(VIII.) supergene mineral phases.

Similarly, Varček [5] states that U-REE mineralization in quartz-fluorapatite veins near Čučma (Gemeric Unit) is accompanied by younger quartz, Fe and Fe-Mg carbonates and a diverse range of sulphidic minerals.

\subsection{Mineralization Type}

In the Gemeric Unit, quartz and quartz-apatite veins with $U$ and U-REE mineralization and accompanying $\mathrm{Au}$ and sulphidic mineralization are widespread in several localities, including the following: Hnilec-Peklisko, Gemerská Poloma-Krátka valley [5,7,33,34], Henclová [4], Čučma-Kutačka vein and other occurrences [1,5,6,8-10,33,34], Betliar [6,9], Prakovce-Zimná voda [7,34-37] and Zlatá Idka [2,6]. Their common feature is the location of the Lower Paleozoic rocks in the vicinity of the intrusions of the Permian Gemeric granites.

According to our own, as well as previous, mineralogical research by Varček [5] or Rojkovič [9], it is possible to divide uranium (and REE)-bearing quartz ( \pm apatite) veins into the following two main types (based on the association of main primary minerals and the content of $\mathrm{P}$ and $\mathrm{Y}+\mathrm{REE})$ :

(A) Hydrothermal quartz-apatite veins containing U-REE mineralization (Betliar, Čučma-Kutačka vein, and some veins in the Majerská valley area) are characterized by a high content of P (0.11-3.47 wt. \%) and Y + REE (up to 1.23 wt. \%). Although Varček [5] reports a rich association of sulphide minerals from the Kutačka vein, this type of mineralization is characterized by a very low to zero sulphide content, as evidenced by the overall low $\mathrm{S}$ content $(<0.04 \mathrm{wt}$. \%; quartz-apatite vein with U-REE mineralization from the Majerská valley; Table 1). Besides quartz and apatite, major primary minerals are represented by $\mathrm{Y}$ - and REE-bearing minerals (such as monazite-(Ce) to monazite-(Nd) and xenotime-( $(\mathrm{Y}))$, uraninite, rutile and titanite $[5,6,8-10,33,34]$. The element association typical of this mineralization type is Si-P-Ca-U-REE. Rojkovič et al. [9] also mentioned increased contents of $\mathrm{Pb}, \mathrm{Mo}$ and $\mathrm{Au}$. Despite the increased Mo content, molybdenite is a rare mineral in microscopic amounts. From other sulphides, pyrite is relatively abundant, and galena was found only as a minor mineral phase (Števko, unpublished data).

(B) Hydrothermal quartz veins (with no or minor apatite content) containing $U$ ( \pm sulphidic)- $( \pm A u)-( \pm R E E)$ mineralization (Hnilec-Peklisko, Gemerská Poloma-Krátka valley, Henclová, Prakovce-Zimná voda and Zlatá Idka). This type of mineralization is characterized by a zero to very low (up to $0.1 \mathrm{wt}$. \%) content of P. The Y + REE content is generally also reduced, compared to the first type of mineralization; their significantly increased content (with an average of $0.17 \mathrm{wt}$. \%) was found only in the Zimná voda locality. Apatite and $Y$ and REE-bearing phases are only accessory minerals. The content of sulphides in this type of mineralization is variable but stable. Besides quartz, the essential primary minerals are represented by uraninite, brannerite (in different quantitative ratios) and rutile, accompanied by tourmaline and rich assemblage of the following sulphidic minerals: Fe-Ni-Co sulphoarsenides, pyrite, chalcopyrite, galena, tetrahedrite, gold, bismuthinite, millerite, sphalerite, pyrrhotite, etc. Molybdenite is very rare in this mineralization type. The typical element association is $\mathrm{Si}-(\mathrm{P})-(\mathrm{Ca})-\mathrm{U}-\mathrm{Ni}-\mathrm{Co}-\mathrm{Fe}-\mathrm{Pb}-\mathrm{Bi}-\mathrm{Au}-\mathrm{Ti}-\mathrm{Zr}-\mathrm{B}$.

The studied quartz veins containing U-Mo mineralization from the Majerská valley can be included in the first approximation to the above-mentioned mineralization type $\mathrm{B}$. However, it is necessary to note some of their peculiarities, as follows:

- Compared to type A, fluorapatite is only a minor component in the studied veins (it only has the character of an accessory mineral), as evidenced by the XRF map of $\mathrm{Ca}$ and $\mathrm{P}$ distribution in the gangue (Figure 3 ) and a significantly reduced content of those elements (see Table 1).

- The most abundant ore minerals are molybdenite and uraninite, accompanied by a diverse (but less represented) assemblage of sulphides (pyrite, galena), sulphos- 
alts (mainly minerals of the kobellite-tintinaite series), sulphoarsenides (cobaltite, gersdorffite, ullmannite), Bi minerals (bismuth, bismuthinite) and sulphotellurides (tetradymite, joséite-A, joséite-B, etc.).

- The typical association of the elements in the studied veins is (the elements in parentheses have a less significance) as follows: $\mathrm{Si}-(\mathrm{P})-(\mathrm{Ca})-\mathrm{U}-\mathrm{Mo}-(\mathrm{Pb})-(\mathrm{Bi})-(\mathrm{Te})$.

Therefore, the significantly reduced $\mathrm{P}$ content and the abundant presence of sulphidic minerals allow for the classification of the studied veins into the mineralization type B. However, they notably differ from this type of mineralization by the abundant presence of molybdenite as well as an elevated content of Te. In the vein U-(REE) mineralization hosted in the Lower Paleozoic rocks of the Gemeric Unit, molybdenite was only rarely found at the Zimná voda locality [36] and Čučma-Kutačka (Števko, unpublished data). In the Gemeric Unit, an abundance of molybdenite, besides uraninite, is a characteristic feature of deposits and occurrences of stratiform, veinlets and stockwork, mainly volcanogenic (or tectonic) U-Mo mineralization hosted in Upper Paleozoic (Permian) rocks of Northern Gemeric (e.g., Novoveská Huta, Košice-Kurišková, Košické Hámre, Matejovce nad Hornádom; [33,34,38]). In general, acidic to intermediate volcanic effusive rocks and their pyroclastics represent a significant source of $U$ and Mo for this type of mineralization $[39,40]$. The most important and widespread volcanogenic mineralization in the world is associated with felsic, acidic volcanics—rhyolites, e.g., McDermitt and Virgin Valley caldera deposits in the USA [41], Ben Lomond and Maureen deposits in Australia [42,43], or Smolian deposit in Bulgaria [44].

On this basis, the studied quartz veins with U-Mo mineralization in Majerská valley near Č učma can be defined as a separate subtype of the above-mentioned hydrothermal vein mineralization of type $B$.

\subsection{Genesis and Age of U-Mo Mineralization}

Quartz and quartz-apatite veins containing $U$ and U-REE mineralization are relatively widespread in the Gemeric Unit; however, their exact dating is still scattered. In addition, there are two fundamentally different views on the genesis of this type of hydrothermal mineralization. Rojkovič [34] and Rojkovič et al. [9,36,45] considered mineralization as Hercynian (although without any dating), whereby the Permian Gemeric granites presumably provided fluids and thermal energy. The hydrothermal effect of granites could have caused a mobilization of P, REE and U from the surrounding Lower Paleozoic graphitic phyllites, metalydites and metaphosphates, and a subsequent concentration of these elements in the hydrothermal veins. However, these authors also admit the possible younger, Alpine age of mineralization. On the contrary, the recent electron-microprobe $\mathrm{U}-\mathrm{Pb}$ uraninite dating from Čučma [10] gave a Paleoalpine age (207 \pm 2 Ma; Late Triassic). On this basis, Permian granites are still considered as a possible source of mineralization elements. However, mobilization of $\mathrm{P}, \mathrm{F}, \mathrm{U}, \mathrm{REE}$ and $\mathrm{Y}$ from granites and their concentration in the hydrothermal veins had to occur during the Late Triassic tectonothermal rejuvenation of the Gemeric Unit [46].

Questions regarding the petrology, geochemistry and age of the Gemeric granites have been solved by several authors. The granitic rocks of the Gemeric Unit represent a distinct type of specialized ( $\mathrm{Sn}-\mathrm{W}-\mathrm{F})$, highly evolved suite with S-type affinity that differs from other granitoids occurring in the Veporic and Tatric Units of the Western Carpathian crystalline basement. They are enriched in fluorine, phosphorus and rare lithophile elements, such as $\mathrm{Li}, \mathrm{Rb}, \mathrm{Cs}, \mathrm{B}, \mathrm{Ga}, \mathrm{Sn}, \mathrm{W}, \mathrm{Nb}$, Ta and U (e.g., [23,47-51] and others). More recent zircon $\mathrm{U}-\mathrm{Pb}$ and molybdenite $\mathrm{Re}-\mathrm{Os}$ isotopic dating, as well as the chemical ages of monazite, indicate emplacement of the Gemeric granites and related post-magmatic mineralization during Late Permian period. More specifically, the age of the Hnilec granites (in the tectonic zone so-called Northern Gemeric hotline) ranges from 289 to $238 \mathrm{Ma}$ (with an average of $262 \mathrm{Ma}$ ) [46,51-54]. The age of the granites emplaced in the Southern Gemeric hotline (Betliar, Elisabeth Mine, Zlatá Idka and Poproč bodies) varies between 303 and $241 \mathrm{Ma}$ (with an average of $266 \mathrm{Ma}$ ) [46,51,53]. 
In the Čučma area, granitic rocks do not reach the surface. The Čučma granite was uncovered by mining works on the Gabriela quartz-stibnite vein, and it was also drilled by borehole Sb-3, $700 \mathrm{~m}$ to the NE of the studied locality, at a depth of about $330 \mathrm{~m}$ below the surface [55].

The chemical in situ electron-microprobe U-Pb dating of uraninite (Table 3) from the studied U-Mo mineralization hosted in a hydrothermal quartz vein, yielded an average age of $265 \mathrm{Ma}$, corresponding to the Guadalupian Epoch of Permian. The data obtained corresponds very well with the age of the Gemeric granites, especially with the age of the nearby Betliar granite intrusion (268-264 Ma; [51]), outcropping less than $3 \mathrm{~km}$ to the NW of the studied locality. The hidden body of the Čucma granite is less than $1 \mathrm{~km}(400-800 \mathrm{~m})$ away from the studied vein. In frame of hydrothermal mineralizations, the age of the studied uraninite (Majerská valley) agrees with the age of molybdenite of pneumatolytic (greisen type) Sn-Mo mineralization (263 $\pm 0.9 \mathrm{Ma}$; [54]), directly genetically related to the Hnilec granite body (Figure 14).

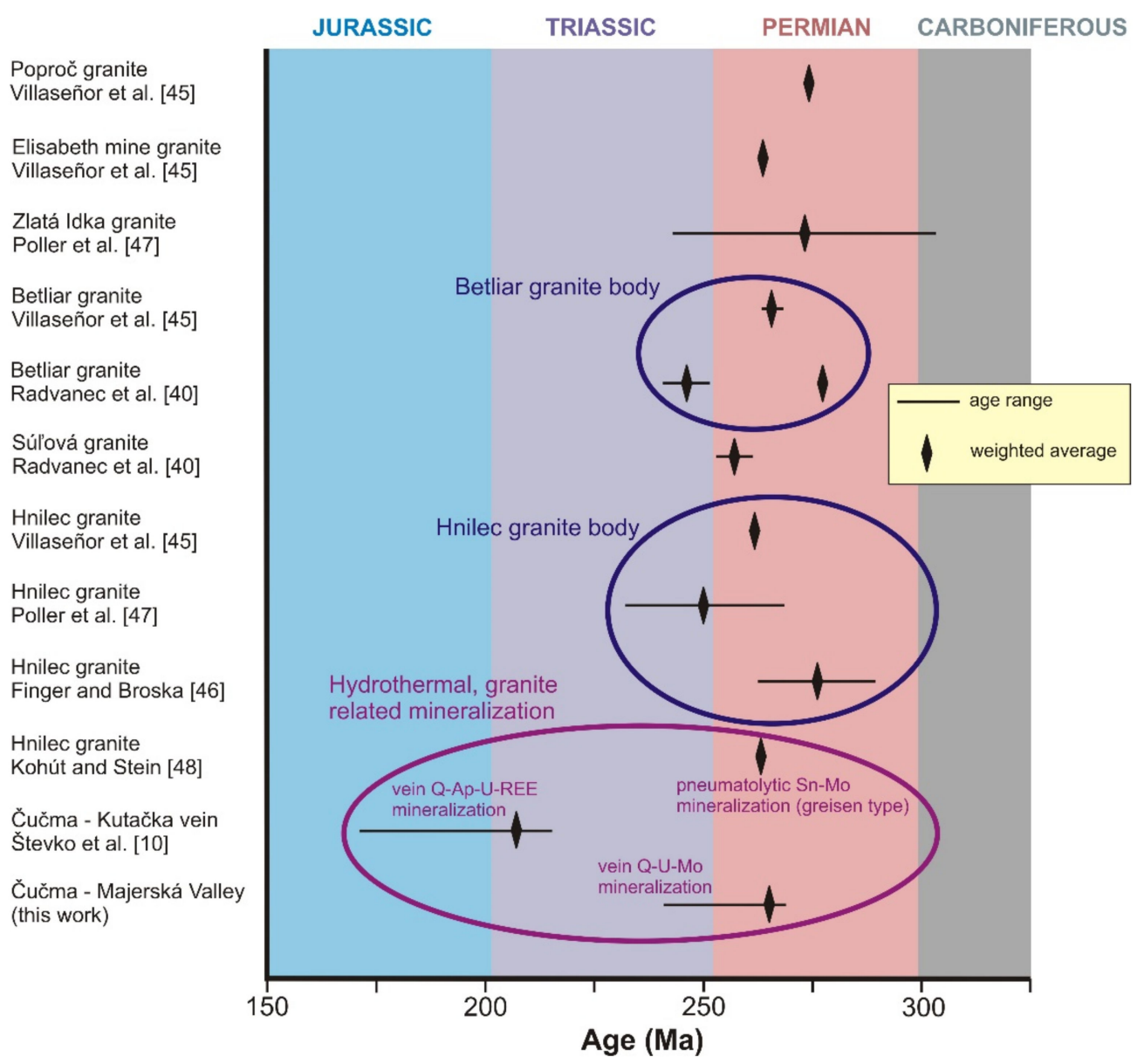

Figure 14. Summary of the age data of the Gemeric granites and associated hydrothermal mineralization. The age boundaries of the stratigraphic periods are plotted according to the ICS [56].

The dating of studied uraninite clearly confirmed its Hercynian age. The correlation of its age with published data about the age of the Gemeric granites [46,51-54] as well as the spatial connection of the studied mineralization with the Čučma granite is clearly pointing to a direct connection between post-collisional S-type granites and the studied U-Mo mineralization. This supports the view of Rojkovič [34] and Rojkovič et al. [9,36,45], that the Gemeric granites were donors of fluids, thermal energy and elements such as $\mathrm{P}$, REE, $\mathrm{U}$, etc. In addition, some fluids were mobilized via a hydrothermal effect of granites from the surrounding Lower Paleozoic metasediments. A number of published works have shown that uraninite is sensitive to subsequent post-mineralization processes (e.g., [57-63]), 
which are also reflected in the results of dating. From this point of view, the younger age of uraninite obtained from the U-REE-bearing quartz-fluorapatite vein at Čučma by Števko et al. [10] (Figure 14) reflect subsequent, Paleoalpine processes and might represent a younger, remobilized generation of uraninite, although the whole mineralization is also originally related to S-type granitic magmatism. Such remobilization of Hercynian, hydrothermal vein $\mathrm{U}$ mineralization in the Gemeric Unit is also supported by the dual, Hercynian and Alpine ages acquired by the chemical dating of uraninite from the Zlatá Idka and Prakovce-Zimná voda occurrences (Ondrejka-unpublished data, Ferenc and Kopáčik - unpublished data).

The classification of the vein uranium deposits (summary in [64]) is based on the presence or absence of other mineral components besides $U$ minerals, also on their geological setting. According to these classifications [65-68], the studied quartz veins with U-Mo and accompanying sulphidic mineralization at the Majerská Valley near Čučma can be qualified as a granite-related (perigranitic) with a complex mineral association (although the representation of other minerals, except uraninite and molybdenite, is inferior).

\section{Conclusions}

Quartz and quartz-apatite veins containing $U$ and U-REE mineralization and the accompanying $\mathrm{Au}$ and sulphidic mineralization are widespread at several localities, hosted in Lower Paleozoic rocks of the Gemeric Unit. Ore mineralization at the studied hydrothermal quartz vein at the Majerská valley near Čučma is mainly represented by uraninite and molybdenite, accompanied by a diverse association of sulphides, sulphosalts and sulphotellurides. The chemical in-situ electron-microprobe $\mathrm{U}-\mathrm{Pb}$ dating of uraninite from the hydrothermal veins yielded an average age of $265 \mathrm{Ma}$, corresponding to the Guadalupian Epoch of Permian; the obtained data correspond with the age of the Gemeric S-type granites, and also with the age of the greisen type Sn-Mo mineralization associated with them. The age correlation of uraninite with Gemeric granites and the spatial connection of the studied mineralization with the Čucrma granite allows us to assume that it is a Hercynian, granite-related (perigranitic) mineralization.

Author Contributions: Conceptualization, Š.F. and M.Š.; methodology, T.M. and S.M.; software, R.K. and E.H.; investigation, Š.F. and M.Š.; data curation, Š.F.; writing—original draft preparation, Š.F.; writing - review and editing, M.Š. and Š.F. All authors have read and agreed to the published version of the manuscript.

Funding: The SLOVAK RESEARCH AND DEVELOPMENT AGENCY under the contract APVV-190065, as well as the MINISTRY OF EDUCATION, SLOVAK REPUBLIC VEGA-1/0237/18 and KEGA 033UMB-42021 projects funded this research.

Data Availability Statement: The data presented in this study are available on request from the corresponding author. The data are not publicly available because all representative data are contained in this work.

Acknowledgments: The authors thank to Patrik Konečný from the State Geological Institute of D. Štúr (Bratislava) for precise dating microprobe analyses of uraninite and reviewers for their precise reviews and improvement of the manuscript.

Conflicts of Interest: The authors declare no conflict of interest. The funders had no role in the design of the study; in the collection, analyses, or interpretation of data; in the writing of the manuscript, or in the decision to publish the results.

\section{References}

1. Šváb, J.; Tulis, J.; Badár, J. Final Report about Results of Geological Survey in Čučma Locality; State Geological Institute of D. Štúr Archive: Bratislava, Slovakia, 1966. (In Slovak)

2. Melnikova, A.M. Short Reports of Mineral Composition of Some Uranium Occurrences in Slovakia; Uranpres Archive: Spišská Nová Ves, Slovakia, 1973. (In Russian)

3. Tréger, M. Occurrences of uranium-bearing phosphates in the Spišsko-gemerské rudohorie Mts. Miner. Slov. 1973, 5, 61-64. (In Slovak) 
4. Varček, C. Mineralogical Research of Vein Uranium Mineralization in the Central Part of the Spišsko-Gemerské Rudohorie Mts; Uranpres Archive: Spišská Nová Ves, Slovakia, 1975. (In Slovak)

5. Varček, C. Some rare types of mineralization in the Spišsko-gemerské rudohorie Mts. In Deposit Forming Processes in the Western Carpathians Conference Proceedings, Bratislava, Slovakia, 29-30 May 2019; Háber, M., Ed.; FNS UK: Bratislava, Slovakia, 1977; pp. 93-99.

6. Donát, A. Evaluation of REE Anomalies Occurrences in the Permian and in the Gelnica Gropup of SGRl; State Geological Institute of D. Štúr Archive: Bratislava, Slovakia, 1998. (In Slovak)

7. Donát, A.; Mihál, F.; Novotný, L. Geological Survey Works on Au in the Older Paleozoic in SGR; State Geological Institute of D. Štúr Archive: Bratislava, Slovakia, 2000. (In Slovak)

8. Rojkovič, I. Minerals of the crandallite series in quartz-apatite vein near Čučma. Miner. Slov. 1993, 25, 151-153. (In Slovak)

9. Rojkovič, I.; Konečný, P.; Novotný, L.; Puškelová, L.; Streško, V. Quartz-apatite-REE vein mineralization in Early Paleozoic rocks of the Gemeric Superunit, Slovakia. Geol. Carpath. 1999, 50, 215-227.

10. Števko, M.; Uher, P.; Ondrejka, M.; Ozdín, D.; Bačík, P. Quartz-apatite-REE phosphates-uraninite vein mineralization near Čučma (eastern Slovakia): A product of early Alpine hydrothermal activity in the Gemeric Superunit, Western Carpathians. J. Geosci. 2014, 59, 209-222. [CrossRef]

11. Ferenc, Š.; Rojkovič, I.; Mato, L. Uranyl minerals of Western Carpathians. In Mineralogy of Bohemian Massif and Western Carpathians; Conference Proceedings; Zimák, J., Ed.; Palacký University: Olomouc, Czech Republic, 2003; pp. 17-23. (In Slovak)

12. Ferenc, Š.; Biroň, A.; Sejkora, J.; Sýkorová, M. Phosphuranylite from the oxidation zone of the vein quartz-apatite-REE-U mineralization at Majerská Valley near Čučma (Slovenské Rudohorie Mts., Gemeric Unit). Bull. Miner. Petrolog. 2017, 25 , 23-32. (In Slovak)

13. Ferenc, Š.; Mikuš, T.; Spišiak, J.; Milovská, S. Supergene minerals in quartz fluorapatite hydrothermal veins with U-Mo and U-REE mineralization near Čučma (Gemeric Unit, Western Carpathians, eastern Slovakia): Preliminary study. In Proceedings from Mineralogical-Petrological Conference Petros; Ondrejka, M., Fridrichová, J., Eds.; FNS UK: Bratislava, Slovakia, 2019 ; pp. 17-19.

14. Mahel, M.; Vozár, J. Contribution to knowledge of Permian and Triassic in the North-Gemeric syncline. Geol. Pr. Spr. 1971, 56, 47-66. (In Slovak)

15. Snopko, L.; Ivanička, J. Considerations on the paleogeography in the Lower Paleozoic of Spišsko-Gemerské Rudohorie Mts. In Paleogeographical Evolution of the Western Carpathians, 1st ed.; Vozár, J., Ed.; Geological Institution of D. Štúr: Bratislava, Slovakia, 1978; pp. 269-280. (In Slovak)

16. Ivanička, J.; Snopko, L.; Snopková, P.; Vozárová, A. Gelnica Group_Lower Unit of Spišsko-Gemerské Rudohorie Mts. (West Carpathians), Early Paleozoic. Geol. Zbor. Geol. Carpath. 1989, 40, 483-501.

17. Vozárová, A. Variscan metamorphism and crustal evolution of the Gemericum. Západ Karpaty. Sér Miner. Petrogr. Geochém. Metalogen. 1993, 16, 55-117. (In Slovak)

18. Putiš, M.; Sergeev, S.; Ondrejka, M.; Larionov, A.; Siman, P.; Spišiak, J.; Uher, P.; Paderin, I. Cambrian-Ordovician metaigneous rocks associated with Cadomian fragments in the West-Carpathian basement dated by SHRIMP on zircons: A record the Gondwana active margin setting. Geol. Carpath. 2008, 59, 3-18.

19. Faryad, S.W. Metamorphism of the Early Paleozoic salic to intermediate volcanic rocks. Miner. Slov. 1991, 23, 325-332. (In Slovak)

20. Faryad, S.W. Metamorphism of the Early Paleozoic sedimentary rocks in Gemericum. Miner. Slov. 1991, 23, 315-324. (In Slovak)

21. Bajaník, Š.; Hanzel, V.; Mello, J.; Pristaš, J.; Reichwalder, P.; Snopko, L.; Vozár, J.; Vozárová, A. Explanation to Geological Map of the Slovenské Rudohorie Mts._Eastern Part, 1:50,000, 1st ed.; State Geological Institute of D. Štúr: Bratislava, Slovakia, 1983; 223p. (In Slovak)

22. Bajaník, Š.; Ivanička, J.; Mello, J.; Reichwalder, P.; Pristaš, J.; Snopko, L.; Vozár, J.; Vozárová, A. Geological Map of the Slovenské Rudohorie Mts.-Eastern Part, 1:50,000, 1st ed.; State Geological Institute of D. Štúr: Bratislava, Slovakia, 1984. (In Slovak)

23. Uher, P.; Broska, I. Post-orogenic Permian granitic rocks in the Western Carpathian-Pannonian area: Geochemistry, mineralogy and evolution. Geol. Carpath. 1996, 47, 311-321.

24. Šefara, J.; Bielik, M.; Vozár, J.; Katona, M.; Szalaiová, V.; Vozárová, A.; Šimonová, B.; Pánisová, J.; Schmidt, S.; Götze, H.J. 3D density modelling of Gemeric granites of the Western Carpathians. Geol. Carpath. 2017, 68, 177-192. [CrossRef]

25. Vozárová, A.; Šarinová, K.; Sergeev, S.; Larionov, A.; Presnyakov, S. Late Cambrian/Ordovician magmatic arc type volcanism in the Southern Gemericum basement, Western Carpathians, Slovakia: U-Pb (SHRIMP) data from zircons. Int. J. Earth Sci. 2010, 99, 17-37. [CrossRef]

26. Vozárová, A.; Rodionov, N.; Šarinová, K.; Presnyakov, S. New zircon ages on the Cambrian-Ordovician volcanism of the Southern Gemericum basement (Western Carpathians, Slovakia): SHRIMP dating, geochemistry and provenance. Int. J. Earth Sci. 2017, 106, 2147-2170. [CrossRef]

27. Grecula, P.; Kobulský, J.; Gazdačko, L.; Németh, Z.; Hraško, L.; Novotný, L.; Maglay, J. Geological Map of the Spišsko-Gemerské Rudohorie Mts., 1:50,000, 1st ed.; State Geological Institute of D. Štúr: Bratislava, Slovakia, 1984. (In Slovak)

28. Grecula, P.; Kobulský, J.; Gazdačko, L.; Németh, Z.; Hraško, L.; Novotný, L.; Maglay, J.; Pramuka, S.; Radvanec, M.; Kucharič, L.; et al. Explanations to Geological Map of the Spišsko-Gemerské Rudohorie Mts., 1:50,000, 1st ed.; State Geological Institute of D. Štúr: Bratislava, Slovakia, 2011; p. 308. (In Slovak)

29. Montel, J.M.; Foret, S.; Veschambre, M.; Nicollet, C.; Provost, A. Electron microprobe dating of monazite. Chem. Geol. 1996, 131, 37-53. [CrossRef] 
30. Pouchou, J.L.; Pichoir, F. "PAP” ( $\varphi \rho Z)$ procedure for improved quantitative microanalysis. In Microbeam Analysis; Armstrong, J.T., Ed.; San Francisco Press: San Francisco, CA, USA, 1985; pp. 104-106.

31. Makovicky, E.; Makovicky, M. Representation of composition in the bismuthinite-aikinite series. Can. Miner. 1978, 16, 405-409.

32. Zakrzewski, M.A.; Makovicky, E. Izoklakeite from Vena, Sweden, and the kobellite homologous series. Can. Miner. 1986, 24, 7-18.

33. Rojkovič, I.; Novotný, L. Uranium mineralization in Gemericum. Miner. Slov. 1993, 25, 368-370. (In Slovak)

34. Rojkovič, I. Uranium Mineralization in Slovakia, 1st ed.; Comenius University: Bratislava, Slovakia, 1997 ; p. 117.

35. Novotný, L.; Čížek, P. New occurrence of uranium and gold, southern from Prakovce in Spišsko-gemerské Rudohoriie Mts. Miner. Slov. 1979, 11, 188-190. (In Slovak)

36. Rojkovič, I.; Háber, M.; Novotný, L. U-Au-Co-Bi-REE mineralization in the Gemeric Unit (Western Carpathians, Slovakia). Geol. Carpath. 1997, 48, 303-313.

37. Novotný, L.; Háber, M.; Križáni, I.; Rojkovič, I.; Mihál, F. Gold in Early Paleozoic rocks in the central part of the Spišsko-gemerské rudohorie Mts. Miner. Slov. 1999, 31, 211-216. (In Slovak)

38. Rojkovič, I. U-Mo-Cu mineralization at Matejovce nad Hornádom. Miner. Slov. 1996, 28, 491-500. (In Slovak)

39. Nash, J.T. Volcanic Uranium Deposits-Geology, Geochemical Processes, and Criteria for Resource Assessment; Open-File Report; U.S. Geological Survey: Reston, VA, USA, 2010.

40. Breit, G.N.; Hall, S.M. Deposit Model for Volcanogenic Uranium Deposits; Open-File Report; U.S. Geological Survey: Reston, VA, USA, 2011.

41. Castor, S.B.; Henry, C.D. Geology, geochemistry, and origin of volcanic rock-hosted uranium deposits in northwestern Nevada and southeastern Oregon, USA. Ore Geol. Rev. 2000, 16, 1-40. [CrossRef]

42. Wilde, A.; Otto, A.; Jory, J.; MacRae, C.; Pownceby, M.; Wilson, N.; Torpy, A. Geology and mineralogy of uranium deposits from Mount Isa, Austarlia: Implications for albitite uranium deposit models. Minerals 2013, 3, 258-283. [CrossRef]

43. McKay, A.D.; Miezitis, Y. Australia's Uranium Resources, Geology and Development of Deposits; AGSO-Geoscienece: Canberra, Australia, 2001; pp. 1-184.

44. Dahlkhamp, F.J. Uranium Deposits of the World; Springer: Berlin/Heidelberg, Germany, 2016; p. 792.

45. Rojkovič, I.; Puškelová, L.; Khun, M.; Medved, J. U-REE-Au in veins and black shales of the Gemericum, Slovakia. In Mineral Deposits: From Their Origin to Their Environmental Impacts; Conference Proceedings; Pašava, J., Kříbek, B., Žák, K., Eds.; Balkema: Rotterdam, The Netherlands, 1995; pp. 789-792.

46. Radvanec, M.; Konečný, P.; Ondrejka, M.; Putiš, M.; Uher, P.; Németh, Z. The Gemeric granites as an indicator of the crustal extension above the Late-Variscan subduction zone during the Early Alpine riftogenesis (Western Carpathians): An interpretation from the monazite and zircon ages dates by CHIME and SHRIMP methods. Miner. Slov. 2009, 41, 381-394. (In Slovak)

47. Broska, I.; Uher, P. Whole-rock chemistry and genetic typology of the West-Carpathian Variscan granites. Geol. Carpath. 2001, 52, 79-90.

48. Kubiš, M.; Broska, I. The role of boron and fluorine in evolved granitic rock systems (on the example of the Hnilec area, Western Carpathians). Geol. Carpath. 2005, 56, 193-204.

49. Kubiš, M.; Broska, I. The granite system near Betliar village (Gemeric Superunit, Western Carpathians): Evolution of a composite silicic reservoir. J. Geosci. 2010, 55, 131-148. [CrossRef]

50. Broska, I.; Kubiš, M. Accessory minerals and evolution of tin-bearing S-type granites in the western segment of the Gemeric Unit (Western Carpathians). Geol. Carpath. 2018, 59, 483-497. [CrossRef]

51. Villaseñor, G.; Catlos, E.J.; Broska, I.; Kohút, M.; Hraško, L.; Aguilera, K.; Etzel, T.M.; Kyle, R.; Stockli, D.F. Evidence for widespread mid-Permian magmatic activity related to rifting following the Variscan orogeny (Western Carpathians). Lithos 2021, 390-391, 106083. [CrossRef]

52. Finger, F.; Broska, I. The Gemeric S-type granites in southeastern Slovakia: Late Palaeozoic or Alpine intrusions? Evidence from electron-microprobe dating of monazite. Schweiz Miner. Petrogr. Mitt. 1999, 79, 439-443.

53. Poller, U.; Uher, P.; Broska, I.; Plašienka, D.; Janák, M. First Permian-Early Triassic zircon ages for tin-bearing granites from the Gemeric unit (Western Carpathians, Slovakia): Connection to the post-collisional extension of the Variscan orogen and S-type granite magmatism. Terra Nova 2002, 14, 41-48. [CrossRef]

54. Kohút, M.; Stein, H. Re-Os molybdenite dating of granite-related Sn-W-Mo mineralisation at Hnilec, Gemeric Superunit, Slovakia. Miner. Petrol. 2005, 85, 117-129. [CrossRef]

55. Pecho, J.; Beňka, J.; Gargulák, M.; Václav, J. Geological Research of Stibnite Deposits in the Betliar-Čučma-Volovec Area; State Geological Institute of D. Štúr Archive: Bratislava, Slovakia, 1981. (In Slovak)

56. Cohen, K.M.; Finney, S.C.; Gibbard, P.L.; Fan, J.X. The ICS International Chronostratigraphic Chart. Episodes 2013, 36, 199-204. [CrossRef] [PubMed]

57. Grandstaff, D.E. A kinetic study of the dissolution of uraninite. Econ. Geol. 1976, 71, 1493-1506. [CrossRef]

58. Finch, R.J.; Ewing, R.C. The corrosion of uraninite under oxidizing conditions. J. Nuclear Mater. 1992, 190, 133-156. [CrossRef]

59. Kotzer, T.G.; Kyser, T.K. O, U, and Pb isotopic and chemical variations in uraninite: Implications for determining the temporal and fluid history of ancient terrains. Am. Miner. 1993, 78, 1262-1274.

60. Alexandre, P.; Kyser, T.K. Effects of cationic substitutions and alteration in uraninite, and implications for the dating of uranium deposits. Can. Miner. 2005, 43, 1005-1017. [CrossRef] 
61. Timón-Sánchez, S.M.; López-Moro, F.J.; Romer Elrhede, D.; Fernández-Fernández, A.; Moro-Benito, C. Late-Variscan multistage hydrothermal processes unveiled by chemical ages coupled with compositional and textural uraninite variations in $\mathrm{W}-\mathrm{Au}$ deposits in the western Spanish Central System Batolith. Geol. Acta 2019, 17.1, 1-19.

62. Yuan, F.; Jiang, S.Y.; Liu, J.; Zhang, S.; Xiao, Z.; Liu, G.; Hu, X. Geochronology and geochemistry of uraninite and coffinite: Insights into ore forming process in the pegmatite-hosted uraniferous province, North Qinling, Central China. Minerals $2019,9,552$. [CrossRef]

63. Corcoran, L.; Simonetti, A. Geochronology of uraninite revisited. Minerals 2020, 10, 205. [CrossRef]

64. Ruzicka, V. Vein uranium deposits. Ore Geol. Rev. 1993, 8, 247-276. [CrossRef]

65. Robinson, P.C. A genetic classification of Canadian uranium deposits. Can. Miner. 1958, 6, 513-521.

66. Lang, A.H.; Griffith, J.W.; Steacy, H.R. Canadian Deposits of Uranium and Thorium; Geological Survey of Canada: Ottawa, ON, Canada, 1962; p. 324.

67. Ruzicka, V. Geological comparison between East Europian and Canadian uranium deposists. Geol. Surv. Can. Pap. 1971, 48-70, $1-195$.

68. Rich, R.A.; Holland, H.D.; Petersen, U. Hydrothermal Uranium Deposits; Elsevier: Amsterdam, The Netherlands, $1977 ;$ p. 264. 